\title{
DETAILED METHODS
}

\section{Energy-Dispersive X-Ray Fluorescence}

Whole-rock samples ( $\mathrm{n}=252)$ were analyzed using standards-based energy-dispersive Xray fluorescence (ED-XRF) at the University of Missouri Research Reactor (Missouri, United States) using an Elva-X table-top spectrometer, operated at $40 \mathrm{kV}$. Calibration curves were established from obsidian source specimens previously analyzed by NAA, inductively-coupled plasma mass spectrometry (ICP-MS), and ED-XRF (Glascock and Ferguson, 2012). The accuracies of elements measured by ED-XRF ranged from $2-4 \%$ of the reported values.

\section{Neutron Activation Analysis}

Descriptions of neutron activation analysis (NAA) procedures and standards are provided by Glascock et al. (2007). Accuracies of individual NAA determinations ranged from 1\% to $10 \%$ of the reported values.

\section{Wavelength-Dispersive X-Ray Fluorescence}

Samples were milled to powder by hand using an agate mortar and pestle, which were cleaned thoroughly with acetone to prevent cross-sample contamination. Next, 0.5 grams of sample were combined with 5.5 grams of lithium meta-tetraborate flux in a platinum crucible and fused into glass discs using a Phoenix ${ }^{\mathrm{TM}}$ fusion machine, which employs a pre-set menu for pre-melting, melting (at $1030^{\circ} \mathrm{C}$ ), and swirling to homogenize each sample, then pours the melt into pre-heated platinum molds to cool.

The glass discs were analyzed for ten major and eight trace elements using a Bruker S4 Pioneer $^{\mathrm{TM}} \mathrm{X}$-Ray Fluorescence Spectrometer at the University of Maine at Farmington (Maine, United States). The S4 Pioneer ${ }^{\mathrm{TM}}$ uses standard wavelength-dispersive X-ray fluorescence (WD $\mathrm{XRF}$ ) methodology with SpectraPlus ${ }^{\mathrm{TM}}$ software to collect and process data. Calibration lines were constructed using USGS reference materials G2, GSP-2, AGV-2, W2a, and BIR-1. Internal lab standards NJQ-2 and SBG-12 were used to monitor accuracy and precision.

\section{Portable Energy-Dispersive X-Ray Fluorescence (P-XRF)}

In October 2012 we analyzed 35 whole-rock Alca source samples in a lab in Arequipa, Peru using a Bruker Tracer III-SD ${ }^{\text {TM }}$ P-XRF. Data were collected at $40 \mathrm{keV}, 14 \mu \mathrm{A}$ at 2048 channels for 300 seconds per sample. Peak intensities for all analyses were normalized to the backscatter Compton peak of Rh. Calibration was established from 40 obsidian source specimens previously analyzed by NAA, ICP-MS, and ED-XRF (Glascock and Ferguson, 2012) and evaluated by Speakman (2012). We collected three different spot measurements on each sample 
and calculated mean RSDs for each element. Mean RSDs for elements of interest include the instrumental uncertainty, plus uncertainty due to sample effects: $\mathrm{Fe}=1.6 \%, \mathrm{Rb}=1.3 \%, \mathrm{Sr}=1.6 \%$, $\mathrm{Zr}=2.0 \%$.

\section{Statistical Cluster Analysis}

To test sample assignments we used Gauss statistical software to conduct i) hierarchical cluster analysis using mean Euclidean distance and average linking on the entire suite of element and oxide data obtained by each geochemical technique, yielding dendrograms, and ii) Mahalanobis distance analysis using a sub-set of elements and oxides obtained by each geochemical technique to yield group membership probabilities for each sample.

Data was log transformed prior to hierarchical cluster analysis. Dendrograms for NAA and P-XRF datasets correspond perfectly with sample assignments to sub-sources on the basis of bivariate plots and geographic sample clustering, but ED-XRF and WD-XRF dendrograms fail to discriminate samples in any logical way with respect to bivariate plots and geographic clustering. This disparity is due to differences in the overall suites of element and oxide data collected by each technique and incorporated in the hierarchical cluster analysis. Many element concentrations in the suites obtained by NAA and P-XRF discriminate the Alca sub-sources relative to the suites obtained by ED-XRF and WD-XRF.

Mahalanobis distance analysis was performed for all geochemical techniques, producing tables of group membership probabilities (Tables SI07-SI10). The analysis takes as input the hypothetical groups defined by bivariate plots, geographic sample clusters, and dendrograms and generates jackknife probabilities for membership of all specimens to each group. Higher jackknife probabilities of group membership are assigned to samples at the furthest distance from the other groups. Computation of the Mahalanobis distance metric requires that groups contain at least two more samples than variables. For each geochemical technique, the elements and oxides chosen were those measured most precisely by each technique and which discriminated subsources most clearly based on bivariate plots (Figure 3). Alca-2 was the only Alca sub-source with insufficient samples for this analysis.

Mahalanobis distance analysis confirms that nearly all samples analyzed with all geochemical techniques were assigned to the correct sub-source:

$$
\begin{aligned}
& \text { ED-XRF - Rb (ppm) vs Sr (ppm), 99.6\% correct }(n=249) \\
& \text { NAA - Eu (ppm) vs Th (ppm), 100\% correct }(n=29) \\
& \text { WD-XRF - TiO2 (wt \%) vs Sr (ppm), 98.4\% correct }(n=62) \\
& \text { P-XRF - Rb (ppm) vs Sr (ppm), 100\% correct }(n=32)
\end{aligned}
$$

In summary, the cluster analysis shows that although a small suite of element and oxide concentrations can be used to discriminate Alca sub-sources, the sub-sources are in fact distinct, and sub-source assignments for analyzed samples are replicable using any of the four techniques. 


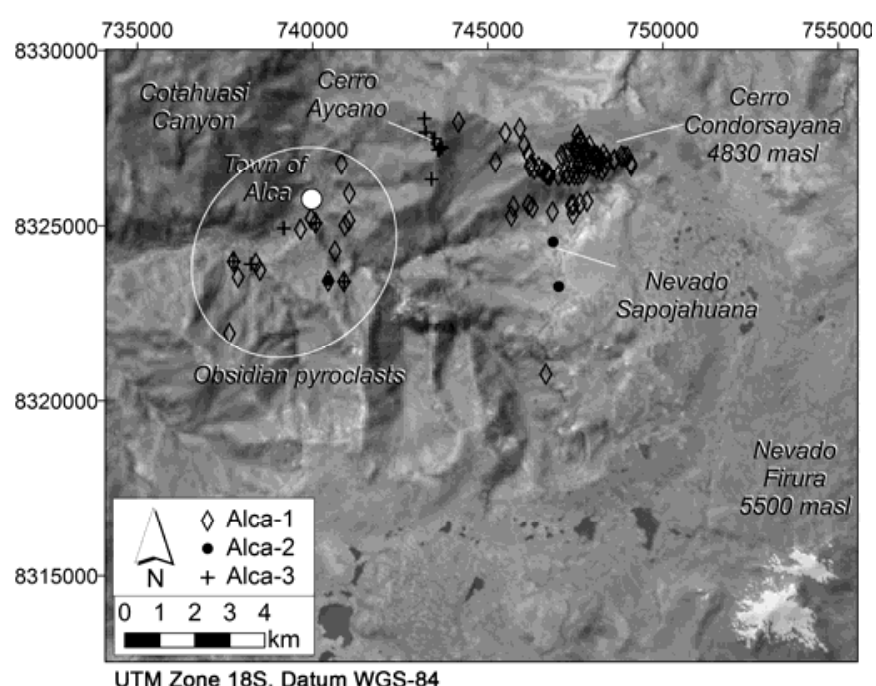

Figure DR1. Detail map of Cotahuasi Canyon area with Alca obsidian samples. Shuttle Radar Topography Mission 90-m digital elevation model and LANDSAT image.

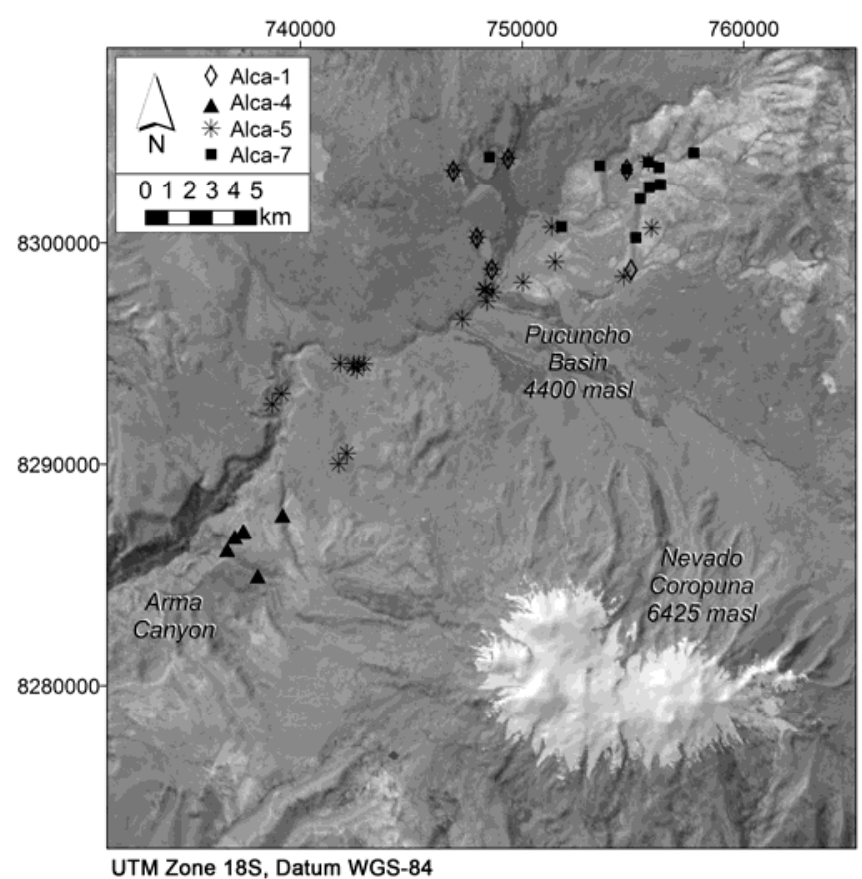

Figure DR2. Detail map of Pucuncho Basin area with Alca obsidian samples. Shuttle Radar Topography Mission 90-m digital elevation model and LANDSAT image. 
Table DR1. Mean concentration data for all techniques

$\begin{array}{cccccccc}\text { ED-XRF } & \text { Alca-1 } & \text { Alca-2 } & \text { Alca-3 } & \text { Alca-4 } & \text { Alca-5 } & \text { Alca-7 } & \begin{array}{c}\text { All Alca } \\ \text { Element }\end{array} \\ \mathbf{n = 1 3 3} & \mathbf{n = 3} & \mathbf{n = 3 0} & \mathbf{n = 1 3} & \mathbf{n = 4 2} & \mathbf{n = 3 1} & \mathbf{n = 2 5 2} \\ \mathrm{K}(\%) & 3.70 \pm 0.22 & 3.79 \pm 0.04 & 3.63 \pm 0.18 & 3.10 \pm 0.10 & 3.62 \pm 0.23 & 3.73 \pm 0.18 & 3.65 \pm 0.25 \\ \mathrm{Ti}(\mathrm{ppm}) & 843 \pm 180 & 1196 \pm 79 & 1385 \pm 93 & 862 \pm 150 & 908 \pm 169 & 1065 \pm 142 & 953 \pm 241 \\ \mathrm{Mn}(\mathrm{ppm}) & 434 \pm 56 & 388 \pm 82 & 387 \pm 58 & 515 \pm 27 & 422 \pm 60 & 349 \pm 48 & 419 \pm 71 \\ \mathrm{Fe}(\mathrm{ppm}) & 5326 \pm 258 & 7037 \pm 87 & 8135 \pm 319 & 5590 \pm 210 & 5243 \pm 269 & 5083 \pm 181 & 5654 \pm 977 \\ \mathrm{Zn}(\mathrm{ppm}) & 39 \pm 3 & 43 \pm 3 & 48 \pm 6 & 33 \pm 2 & 39 \pm 5 & 33 \pm 5 & 39 \pm 6 \\ \mathrm{Ga}(\mathrm{ppm}) & 17 \pm 2 & 16 \pm 1 & 15 \pm 2 & 15 \pm 4 & 15 \pm 2 & 17 \pm 1 & 16 \pm 2 \\ \mathrm{Rb}(\mathrm{ppm}) & 133 \pm 4 & 139 \pm 5 & 129 \pm 5 & 103 \pm 3 & 128 \pm 5 & 159 \pm 4 & 133 \pm 12 \\ \mathrm{Sr}(\mathrm{ppm}) & 84 \pm 9 & 150 \pm 7 & 236 \pm 16 & 169 \pm 12 & 130 \pm 11 & 151 \pm 6 & 123 \pm 52 \\ \mathrm{Y}(\mathrm{ppm}) & 13 \pm 2 & 16 \pm 3 & 15 \pm 2 & 11 \pm 2 & 13 \pm 2 & 15 \pm 1 & 13 \pm 2 \\ \mathrm{Zr}(\mathrm{ppm}) & 100 \pm 10 & 145 \pm 10 & 160 \pm 11 & 95 \pm 9 & 97 \pm 9 & 97 \pm 6 & 107 \pm 22 \\ \mathrm{Nb}(\mathrm{ppm}) & 13 \pm 2 & 13 \pm 2 & 12 \pm 1 & 12 \pm 3 & 14 \pm 3 & 13 \pm 2 & 13 \pm 2\end{array}$

\begin{tabular}{|c|c|c|c|c|c|c|c|}
\hline $\begin{array}{c}\text { NAA } \\
\text { Element } \\
\text { short-count }\end{array}$ & $\begin{array}{l}\text { Alca-1 } \\
n=10\end{array}$ & $\begin{array}{c}\text { Alca-2 } \\
n=2\end{array}$ & $\begin{array}{c}\text { Alca-3 } \\
n=5\end{array}$ & $\begin{array}{c}\text { Alca-4 } \\
n=5\end{array}$ & $\begin{array}{c}\text { Alca-5 } \\
n=9\end{array}$ & $\begin{array}{c}\text { Alca-7 } \\
n=2\end{array}$ & $\begin{array}{c}\text { All Alca } \\
n=33\end{array}$ \\
\hline $\mathrm{Na}(\%)$ & $3.08 \pm 0.08$ & $3.30 \pm 0.09$ & $3.25 \pm 0.05$ & $3.18 \pm 0.02$ & $3.14 \pm 0.06$ & $2.89 \pm 0.04$ & $3.14 \pm 0.11$ \\
\hline $\mathrm{Al}(\%)$ & $7.13 \pm 0.31$ & $7.87 \pm 0.42$ & $7.56 \pm 0.35$ & $7.14 \pm 0.17$ & $7.22 \pm 0.18$ & $6.61 \pm 0.07$ & $7.23 \pm 0.36$ \\
\hline $\mathrm{Cl}(\mathrm{ppm})$ & $542 \pm 70$ & $534 \pm 110$ & $599 \pm 34$ & $404 \pm 61$ & $538 \pm 68$ & $414 \pm 0$ & $520 \pm 87$ \\
\hline $\mathrm{K}(\%)$ & $3.64 \pm 0.24$ & $3.32 \pm 0.03$ & $3.27 \pm 0.18$ & $3.05 \pm 0.13$ & $3.56 \pm 0.16$ & $3.33 \pm 0.31$ & $3.44 \pm 0.28$ \\
\hline Dy (ppm) & $1.99 \pm 0.27$ & $1.99 \pm 0.07$ & $2.05 \pm 0.33$ & $1.63 \pm 0.22$ & $1.96 \pm 0.20$ & $2.12 \pm 0.33$ & $1.94 \pm 0.27$ \\
\hline \multicolumn{8}{|l|}{ long-count } \\
\hline Sc (ppm) & $1.78 \pm 0.02$ & $2.01 \pm 0.01$ & $1.86 \pm 0.02$ & $1.83 \pm 0.02$ & $1.76 \pm 0.03$ & $1.85 \pm 0.00$ & $1.81 \pm 0.07$ \\
\hline $\mathrm{Mn}(\mathrm{ppm})$ & $473 \pm 7$ & $443 \pm 2$ & $561 \pm 6$ & $552 \pm 3$ & $480 \pm 4$ & $447 \pm 1$ & $497 \pm 42$ \\
\hline Fe (ppm) & $5431 \pm 48$ & $7398 \pm 173$ & $8053 \pm 81$ & $5838 \pm 172$ & $5362 \pm 166$ & $4970 \pm 16$ & $5962 \pm 1038$ \\
\hline Co (ppm) & $0.27 \pm 0.06$ & $0.43 \pm 0.03$ & $0.53 \pm 0.06$ & $0.39 \pm 0.06$ & $0.14 \pm 0.02$ & $0.33 \pm 0.01$ & $0.31 \pm 0.14$ \\
\hline Zn (ppm) & $43 \pm 2$ & $47 \pm 3$ & $46 \pm 1$ & $37 \pm 2$ & $42 \pm 2$ & $32 \pm 1$ & $42 \pm 4$ \\
\hline $\mathrm{Rb}(\mathrm{ppm})$ & $138 \pm 2$ & $145 \pm 0$ & $128 \pm 2$ & $107 \pm 1$ & $131 \pm 2$ & $159 \pm 0$ & $132 \pm 13$ \\
\hline Sr (ppm) & $92 \pm 7$ & $181 \pm 1$ & $270 \pm 7$ & $184 \pm 10$ & $134 \pm 10$ & $160 \pm 15$ & $154 \pm 61$ \\
\hline $\mathrm{Zr}(\mathrm{ppm})$ & $113 \pm 5$ & $170 \pm 5$ & $164 \pm 7$ & $100 \pm 2$ & $112 \pm 9$ & $113 \pm 13$ & $122 \pm 5$ \\
\hline $\mathrm{Sb}(\mathrm{ppm})$ & $0.16 \pm 0.01$ & $0.13 \pm 0.00$ & $0.27 \pm 0.01$ & $0.39 \pm 0.01$ & $0.16 \pm 0.00$ & $0.35 \pm 0.00$ & $0.22 \pm 0.09$ \\
\hline Cs (ppm) & $2.81 \pm 0.02$ & $2.78 \pm 0.02$ & $3.43 \pm 0.05$ & $2.70 \pm 0.01$ & $2.82 \pm 0.06$ & $4.98 \pm 0.00$ & $3.02 \pm 0.56$ \\
\hline $\mathrm{Ba}(\mathrm{ppm})$ & $999 \pm 11$ & $979 \pm 7$ & $1026 \pm 15$ & $1024 \pm 8$ & $1014 \pm 18$ & $819 \pm 10$ & $999 \pm 50$ \\
\hline Lu (ppm) & $0.16 \pm 0.01$ & $0.22 \pm 0.01$ & $0.19 \pm 0.01$ & $0.17 \pm 0.03$ & $0.15 \pm 0.00$ & $0.18 \pm 0.00$ & $0.17 \pm 0.03$ \\
\hline $\mathrm{Hf}(\mathrm{ppm})$ & $3.57 \pm 0.07$ & $4.89 \pm 0.11$ & $4.67 \pm 0.07$ & $3.04 \pm 0.04$ & $3.31 \pm 0.13$ & $3.05 \pm 0.02$ & $3.64 \pm 0.62$ \\
\hline Ta (ppm) & $0.95 \pm 0.01$ & $0.96 \pm 0.00$ & $0.90 \pm 0.00$ & $0.67 \pm 0.00$ & $0.93 \pm 0.02$ & $1.17 \pm 0.00$ & $0.91 \pm 0.12$ \\
\hline La (ppm) & $29.1 \pm 1.0$ & $43.8 \pm 0.0$ & $37.0 \pm 0.5$ & $23.7 \pm 0.2$ & $31.4 \pm 1.7$ & $30.6 \pm 0.1$ & $31.1 \pm 5.1$ \\
\hline $\mathrm{Ce}(p p m)$ & $58.5 \pm 1.6$ & $82.4 \pm 0.2$ & $69.6 \pm 0.8$ & $46.2 \pm 0.4$ & $62.3 \pm 3.0$ & $57.1 \pm 0.0$ & $60.7 \pm 9.0$ \\
\hline $\mathrm{Nd}(\mathrm{ppm})$ & $19.1 \pm 1.0$ & $25.3 \pm 1.7$ & $22.7 \pm 0.8$ & $15.4 \pm 1.0$ & $20.8 \pm 0.9$ & $18.3 \pm 2.1$ & $19.9 \pm 2.8$ \\
\hline Sm (ppm) & $3.57 \pm 0.54$ & $4.26 \pm 0.02$ & $3.83 \pm 0.05$ & $2.78 \pm 0.03$ & $3.64 \pm 0.07$ & $3.38 \pm 0.02$ & $3.54 \pm 0.38$ \\
\hline Eu (ppm) & $0.50 \pm 0.01$ & $0.65 \pm 0.02$ & $0.69 \pm 0.01$ & $0.48 \pm 0.01$ & $0.57 \pm 0.02$ & $0.47 \pm 0.01$ & $0.55 \pm 0.08$ \\
\hline $\mathrm{Tb}(\mathrm{ppm})$ & $0.36 \pm 0.01$ & $0.37 \pm 0.01$ & $0.38 \pm 0.01$ & $0.30 \pm 0.01$ & $0.35 \pm 0.01$ & $0.35 \pm 0.00$ & $0.35 \pm 0.03$ \\
\hline $\mathrm{Yb}(\mathrm{ppm})$ & $1.11 \pm 0.03$ & $1.28 \pm 0.04$ & $1.32 \pm 0.05$ & $0.99 \pm 0.01$ & $1.08 \pm 0.03$ & $1.28 \pm 0.00$ & $1.14 \pm 0.11$ \\
\hline Th (ppm) & $13.9 \pm 0.1$ & $16.5 \pm 0.0$ & $13.7 \pm 0.1$ & $8.0 \pm 0.0$ & $13.8 \pm 0.2$ & $13.3 \pm 2.5$ & $17.1 \pm 0.1$ \\
\hline U (ppm) & $3.42 \pm 0.16$ & $4.02 \pm 0.02$ & $3.34 \pm 0.12$ & $2.13 \pm 0.22$ & $3.61 \pm 0.09$ & $4.82 \pm 0.13$ & $3.39 \pm 0.66$ \\
\hline
\end{tabular}


Table DR1. Mean concentration data for all techniques

\begin{tabular}{|c|c|c|c|c|c|c|c|}
\hline $\begin{array}{c}\text { WD-XRF } \\
\text { Element/Oxide }\end{array}$ & $\begin{array}{c}\text { Alca-1 } \\
n=19\end{array}$ & $\begin{array}{c}\text { Alca-2 } \\
n=3\end{array}$ & $\begin{array}{c}\text { Alca-3 } \\
n=11\end{array}$ & $\begin{array}{c}\text { Alca-4 } \\
n=7\end{array}$ & $\begin{array}{l}\text { Alca-5 } \\
n=17\end{array}$ & $\begin{array}{c}\text { Alca-7 } \\
n=9\end{array}$ & $\begin{array}{c}\text { All Alca } \\
n=66\end{array}$ \\
\hline $\mathrm{Na}_{2} \mathrm{O}$ (wt \%) & $4.3 \pm 0.1$ & $4.5 \pm 0.0$ & $4.5 \pm 0.1$ & $4.4 \pm 0.0$ & $4.2 \pm 0.1$ & $3.9 \pm 0.0$ & $4.3 \pm 0.2$ \\
\hline $\mathrm{MgO}(\mathrm{wt} \%)$ & $0.11 \pm 0.01$ & $0.18 \pm 0.02$ & $0.23 \pm 0.02$ & $0.18 \pm 0.01$ & $0.10 \pm 0.01$ & $0.10 \pm 0.01$ & $0.14 \pm 0.05$ \\
\hline $\mathrm{SiO}_{2}(\mathrm{wt} \%)$ & $70.9 \pm 2.4$ & $73.7 \pm 0.2$ & $71.6 \pm 2.3$ & $72.6 \pm 3.2$ & $75.5 \pm 0.03$ & $74.4 \pm 2.6$ & $73.0 \pm 2.8$ \\
\hline $\mathrm{Al}_{2} \mathrm{O}_{3}(\mathrm{wt} \%)$ & $12.6 \pm 0.3$ & $13.7 \pm 0.1$ & $13.9 \pm 0.3$ & $13.2 \pm 0.3$ & $13.4 \pm 0.1$ & $12.9 \pm 0.3$ & $13.2 \pm 0.5$ \\
\hline $\mathrm{P}_{2} \mathrm{O}_{5}(\mathrm{wt} \%)$ & $0.027 \pm 0.002$ & $0.034 \pm 0.002$ & $0.046 \pm 0.004$ & $0.041 \pm 0.002$ & $0.026 \pm 0.002$ & $0.021 \pm 0.002$ & $0.031 \pm 0.009$ \\
\hline $\mathrm{K}_{2} \mathrm{O}(w t \%)$ & $4.4 \pm 0.1$ & $4.3 \pm 0.0$ & $4.1 \pm 0.0$ & $3.8 \pm 0.0$ & $4.4 \pm 0.1$ & $4.3 \pm 0.0$ & $4.3 \pm 0.2$ \\
\hline $\mathrm{CaO}$ (wt \%) & $0.62 \pm 0.01$ & $0.88 \pm 0.02$ & $1.17 \pm 0.02$ & $0.91 \pm 0.01$ & $0.75 \pm 0.02$ & $0.90 \pm 0.01$ & $0.82 \pm 0.19$ \\
\hline $\mathrm{TiO}_{2}$ (wt \%) & $0.128 \pm 0.003$ & $0.189 \pm 0.004$ & $0.196 \pm 0.017$ & $0.127 \pm 0.004$ & $0.113 \pm 0.004$ & $0.099 \pm 0.003$ & $0.134 \pm 0.034$ \\
\hline $\mathrm{Cr}(\mathrm{ppm})$ & $6 \pm 5$ & $7 \pm 5$ & $11 \pm 5$ & $8 \pm 5$ & $6 \pm 5$ & $7 \pm 4$ & $7 \pm 5$ \\
\hline $\mathrm{MnO}$ (wt \%) & $0.062 \pm 0.001$ & $0.058 \pm 0.003$ & $0.072 \pm 0.004$ & $0.073 \pm 0.002$ & $0.062 \pm 0.001$ & $0.059 \pm 0.001$ & $0.064 \pm 0.006$ \\
\hline $\mathrm{Fe}_{2} \mathrm{O}_{3}(w \mathrm{t} \%)$ & $0.93 \pm 0.05$ & $1.20 \pm 0.02$ & $1.33 \pm 0.04$ & $1.04 \pm 0.14$ & $0.89 \pm 0.05$ & $0.83 \pm 0.07$ & $1.00 \pm 0.18$ \\
\hline Zn (ppm) & $39 \pm 6$ & $36 \pm 2$ & $41 \pm 3$ & $32 \pm 3$ & $34 \pm 2$ & $23 \pm 5$ & $35 \pm 7$ \\
\hline $\mathrm{Rb}(\mathrm{ppm})$ & $154 \pm 5$ & $150 \pm 8$ & $134 \pm 8$ & $117 \pm 5$ & $132 \pm 4$ & $166 \pm 10$ & $143 \pm 16$ \\
\hline $\mathrm{Sr}(\mathrm{ppm})$ & $81 \pm 2$ & $157 \pm 3$ & $222 \pm 4$ & $157 \pm 3$ & $117 \pm 4$ & $139 \pm 3$ & $133 \pm 48$ \\
\hline $\mathrm{Y}(\mathrm{ppm})$ & $14 \pm 2$ & $13 \pm 2$ & $15 \pm 2$ & $12 \pm 2$ & $12 \pm 2$ & $14 \pm 2$ & $13 \pm 2$ \\
\hline $\mathrm{Zr}(\mathrm{ppm})$ & $102 \pm 7$ & $146 \pm 3$ & $155 \pm 14$ & $88 \pm 9$ & $75 \pm 4$ & $74 \pm 6$ & $101 \pm 31$ \\
\hline $\mathrm{Nb}(\mathrm{ppm})$ & $14 \pm 1$ & $13 \pm 1$ & $12 \pm 2$ & $9 \pm 1$ & $11 \pm 1$ & $13 \pm 2$ & $12 \pm 2$ \\
\hline $\mathrm{Ba}(\mathrm{ppm})$ & $1070 \pm 22$ & $1046 \pm 24$ & $1110 \pm 27$ & $1108 \pm 25$ & $1091 \pm 27$ & $886 \pm 19$ & $1060 \pm 75$ \\
\hline
\end{tabular}

P-XRF

Element

$\mathrm{Mn}$ (ppm)

$\mathrm{Fe}$ (ppm)

Zn (ppm)

$\mathrm{Ga}$ (ppm)

$\mathrm{Rb}$ (ppm)

$\mathrm{Sr}$ (ppm)

Y (ppm)

$\mathrm{Zr}$ (ppm)

$\mathrm{Nb}$ (ppm)

Th (ppm)

$\begin{array}{cc}\text { Alca-1 } & \text { Alca-2 } \\ \mathbf{n}=\mathbf{6} & \mathbf{n}=\mathbf{3} \\ 458 \pm 12 & 447 \pm 15 \\ 5614 \pm 124 & 7624 \pm 592 \\ 46 \pm 1 & 49 \pm 1 \\ 17 \pm 1 & 18 \pm 1 \\ 134 \pm 1 & 142 \pm 1 \\ 72 \pm 1 & 136 \pm 4 \\ 14 \pm 1 & 15 \pm 0 \\ 100 \pm 2 & 155 \pm 3 \\ 11 \pm 0 & 12 \pm 0 \\ 13 \pm 0 & 15 \pm 0\end{array}$

Alca-4
$\mathbf{n}=6$
$538 \pm 14$
$5918 \pm 43$
$41 \pm 1$
$17 \pm 1$
$106 \pm 1$
$141 \pm 2$
$13 \pm 0$
$94 \pm 3$
$9 \pm 0$
$8 \pm 1$

$\begin{array}{cc}\text { Alca-7 } & \text { All Alca } \\ \mathbf{n}=\mathbf{7} & \mathbf{n}=\mathbf{3 5} \\ 448 \pm 13 & 491 \pm 42 \\ 5215 \pm 68 & 6228 \pm 1108 \\ 36 \pm 2 & 45 \pm 6 \\ 19 \pm 1 & 18 \pm 2 \\ 157 \pm 2 & 133 \pm 16 \\ 127 \pm 1 & 133 \pm 42 \\ 15 \pm 0 & 15 \pm 1 \\ 86 \pm 1 & 110 \pm 29 \\ 12 \pm 0 & 11 \pm 1 \\ 16 \pm 1 & 13 \pm 3\end{array}$


Table DR2. ED-XRF concentration data

\begin{tabular}{|c|c|c|c|c|c|c|c|c|c|c|c|c|c|c|}
\hline ANID & Sub-source & Date & $\mathrm{K}(\mathrm{ppm})$ & $\mathrm{Ca}(\mathrm{ppm})$ & $\mathrm{Ti}(\mathrm{ppm})$ & $\mathrm{Mn}(\mathrm{ppm})$ & $\mathrm{Fe}(\mathrm{ppm})$ & $\mathrm{Zn}(\mathrm{ppm})$ & $\mathrm{Ga}(\mathrm{ppm})$ & $\mathrm{Rb}(\mathrm{ppm})$ & $\mathrm{Sr}(\mathrm{ppm})$ & $Y(p p m)$ & $\mathrm{Zr}(\mathrm{ppm})$ & $\mathrm{Nb}$ (ppm) \\
\hline KRA001 & ALCA-1 & 11/20/2007 & 35561 & 4103 & 833 & 482 & 5260 & 41 & 18 & 131 & 86 & 12 & 98 & 12 \\
\hline KRA002 & ALCA-1 & 11/20/2007 & 37747 & 4348 & 788 & 495 & 5461 & 41 & 17 & 138 & 85 & 16 & 102 & 13 \\
\hline KRA005 & ALCA-1 & 11/20/2007 & 38409 & 4199 & 804 & 470 & 5366 & 41 & 17 & 133 & 86 & 14 & 97 & 13 \\
\hline KRA006 & ALCA-1 & $11 / 20 / 2007$ & 38187 & 4306 & 763 & 485 & 5440 & 41 & 13 & 131 & 68 & 12 & 95 & 11 \\
\hline KRA007 & ALCA-1 & 11/20/2007 & 36331 & 3860 & 717 & 453 & 5272 & 40 & 17 & 129 & 74 & 11 & 95 & 12 \\
\hline KRA008 & ALCA-1. & $11 / 20 / 2007$ & 39249 & 4170 & 788 & 474 & 5668 & 41 & 19 & 135 & 86 & 13 & 98 & 14 \\
\hline KRA009 & ALCA-1 & $11 / 20 / 2007$ & 36535 & 4177 & 758 & 469 & 5354 & 40 & 17 & 134 & 80 & 12 & 98 & 12 \\
\hline KRA014 & ALCA-1. & $11 / 20 / 2007$ & 33988 & 4022 & 724 & 434 & 5228 & 39 & 16 & 130 & 79 & 13 & 91 & 11 \\
\hline KRA015 & ALCA-1 & 11/20/2007 & 39527 & 4476 & 743 & 499 & 5412 & 40 & 17 & 136 & 79 & 13 & 95 & 13 \\
\hline KRA016 & ALCA-1 & 11/20/2007 & 29837 & 3659 & 711 & 480 & 5068 & 38 & 15 & 130 & 76 & 13 & 94 & 10 \\
\hline KRA017 & ALCA-1 & $11 / 20 / 2007$ & 37112 & 4082 & 707 & 480 & 5295 & 39 & 16 & 132 & 77 & 13 & 93 & 12 \\
\hline KRA018 & ALCA-1 & $11 / 20 / 2007$ & 37661 & 4014 & 683 & 465 & 5299 & 39 & 16 & 135 & 71 & 12 & 93 & 12 \\
\hline KRA019 & ALCA-1 & 11/20/2007 & 34402 & 3956 & 681 & 464 & 5086 & 36 & 18 & 128 & 80 & 12 & 87 & 12 \\
\hline KRA020 & ALCA-1 & $11 / 20 / 2007$ & 37653 & 4301 & 770 & 444 & 5416 & 40 & 17 & 130 & 81 & 12 & 99 & 13 \\
\hline KRA021 & ALCA-1 & $11 / 20 / 2007$ & 36470 & 3824 & 685 & 482 & 5234 & 41 & 13 & 134 & 71 & 12 & 94 & 11 \\
\hline KRA022 & ALCA-1 & $11 / 20 / 2007$ & 37113 & 4290 & 711 & 451 & 5290 & 41 & 13 & 134 & 70 & 13 & 94 & 11 \\
\hline KRA023 & ALCA-1. & $11 / 20 / 2007$ & 40812 & 4640 & 860 & 509 & 5530 & 46 & 20 & 137 & 88 & 14 & 102 & 14 \\
\hline KRA024 & ALCA-1 & 11/20/2007 & 36892 & 3991 & 703 & 468 & 5234 & 39 & 17 & 132 & 79 & 12 & 96 & 12 \\
\hline KRA025 & ALCA-1 & $11 / 20 / 2007$ & 35619 & 3883 & 774 & 438 & 5225 & 39 & 21 & 128 & 88 & 11 & 95 & 13 \\
\hline KRA026 & ALCA-1 & 11/20/2007 & 38176 & 4132 & 746 & 451 & 5344 & 42 & 15 & 132 & 72 & 12 & 95 & 12 \\
\hline KRA027 & ALCA-1 & 11/20/2007 & 35206 & 3862 & 794 & 433 & 5567 & 39 & 17 & 133 & 85 & 13 & 95 & 13 \\
\hline KRA028 & ALCA-1 & $11 / 20 / 2007$ & 37147 & 3726 & 749 & 462 & 5235 & 40 & 16 & 131 & 76 & 10 & 93 & 12 \\
\hline KRA029 & ALCA-1 & $11 / 20 / 2007$ & 35419 & 3908 & 669 & 444 & 5136 & 36 & 16 & 132 & 82 & 13 & 91 & 12 \\
\hline KRA030 & ALCA-1 & 11/20/2007 & 37802 & 4041 & 770 & 478 & 5367 & 40 & 16 & 132 & 80 & 12 & 93 & 12 \\
\hline KRA031 & ALCA-1 & $11 / 28 / 2007$ & 36484 & 4043 & 853 & 501 & 5200 & 40 & 19 & 133 & 95 & 12 & 99 & 13 \\
\hline KRA032 & ALCA-1 & $11 / 28 / 2007$ & 37747 & 4183 & 790 & 491 & 5282 & 42 & 17 & 135 & 78 & 12 & 103 & 12 \\
\hline KRA033 & ALCA-1 & $11 / 28 / 2007$ & 38959 & 4242 & 790 & 469 & 5523 & 41 & 15 & 136 & 82 & 14 & 94 & 13 \\
\hline KRA034 & ALCA-1. & $11 / 28 / 2007$ & 35954 & 3718 & 750 & 467 & 5237 & 38 & 19 & 129 & 81 & 11 & 102 & 12 \\
\hline KRA035 & ALCA-1 & 11/28/2007 & 37548 & 4228 & 840 & 492 & 5369 & 42 & 20 & 131 & 91 & 14 & 100 & 13 \\
\hline KRA036 & ALCA-1 & $11 / 28 / 2007$ & 34406 & 3747 & 784 & 470 & 5135 & 39 & 20 & 128 & 83 & 11 & 95 & 12 \\
\hline KRA037 & ALCA-1 & 11/28/2007 & 33480 & 3656 & 683 & 475 & 4997 & 34 & 15 & 128 & 77 & 12 & 94 & 11 \\
\hline KRA038 & ALCA- 6 & $11 / 28 / 2007$ & 32001 & 3354 & 668 & 434 & 4768 & 35 & 16 & 126 & 76 & 10 & 89 & 11 \\
\hline KRA039 & ALCA-1 & $11 / 28 / 2007$ & 37403 & 4298 & 708 & 453 & 5278 & 38 & 14 & 133 & 74 & 12 & 93 & 12 \\
\hline KRA040 & ALCA-1 & $11 / 28 / 2007$ & 37587 & 3981 & 769 & 442 & 5230 & 41 & 20 & 130 & 87 & 11 & 94 & 13 \\
\hline KRA041 & ALCA-6 & $11 / 28 / 2007$ & 30127 & 3233 & 549 & 396 & 4404 & 32 & 13 & 121 & 63 & 10 & 84 & 9 \\
\hline KRA042 & ALCA-1 & $11 / 28 / 2007$ & 37678 & 3940 & 672 & 460 & 5092 & 36 & 14 & 133 & 77 & 12 & 90 & 12 \\
\hline KRA043 & ALCA-1 & $11 / 28 / 2007$ & 35656 & 3706 & 723 & 441 & 4936 & 39 & 17 & 130 & 80 & 12 & 90 & 12 \\
\hline KRA044 & ALCA-1 & $11 / 28 / 2007$ & 37118 & 4125 & 774 & 495 & 5280 & 40 & 14 & 133 & 75 & 12 & 94 & 11 \\
\hline KRA045 & ALCA-1 & $11 / 28 / 2007$ & 37314 & 3833 & 703 & 462 & 5212 & 39 & 17 & 130 & 78 & 11 & 90 & 12 \\
\hline KRA046 & ALCA-6 & $11 / 28 / 2007$ & 31336 & 3313 & 590 & 439 & 4412 & 32 & 19 & 119 & 74 & 9 & 82 & 11 \\
\hline KRA047 & ALCA-1 & $11 / 28 / 2007$ & 38953 & 4219 & 791 & 482 & 5431 & 42 & 20 & 129 & 81 & 12 & 94 & 13 \\
\hline KRA048 & ALCA-1 & $11 / 28 / 2007$ & 36533 & 4064 & 729 & 449 & 5320 & 41 & 11 & 136 & 68 & 15 & 96 & 11 \\
\hline KRA049 & ALCA-1 & $11 / 28 / 2007$ & 37929 & 4236 & 721 & 437 & 5384 & 39 & 16 & 134 & 78 & 12 & 92 & 13 \\
\hline KRA050 & ALCA-1 & 11/28/2007 & 37164 & 3950 & 770 & 447 & 5220 & 40 & 15 & 134 & 73 & 12 & 95 & 12 \\
\hline KRA051 & ALCA-1 & 11/28/2007 & 38261 & 4275 & 705 & 493 & 5150 & 39 & 16 & 131 & 76 & 12 & 92 & 12 \\
\hline KRA052 & ALCA-1 & 11/28/2007 & 37053 & 4178 & 742 & 454 & 5282 & 41 & 16 & 131 & 80 & 13 & 100 & 12 \\
\hline KRA053 & ALCA-1 & 11/28/2007 & 34749 & 3998 & 734 & 462 & 5099 & 38 & 16 & 128 & 82 & 17 & 93 & 12 \\
\hline KRA054 & ALCA-1. & $11 / 28 / 2007$ & 39124 & 4578 & 754 & 491 & 5412 & 41 & 18 & 136 & 84 & 14 & 96 & 13 \\
\hline KRA055 & ALCA-1 & 11/28/2007 & 36800 & 4173 & 762 & 432 & 5337 & 39 & 17 & 134 & 83 & 13 & 97 & 13 \\
\hline KRA056 & ALCA-1 & $11 / 28 / 2007$ & 37013 & 4226 & 656 & 469 & 5354 & 38 & 15 & 133 & 70 & 13 & 91 & 12 \\
\hline KRA057 & ALCA-1 & $11 / 28 / 2007$ & 37651 & 4027 & 724 & 482 & 5382 & 40 & 15 & 136 & 75 & 13 & 95 & 12 \\
\hline KRA058 & ALCA-1 & $11 / 28 / 2007$ & 38901 & 3979 & 755 & 501 & 5331 & 42 & 16 & 137 & 83 & 14 & 98 & 13 \\
\hline KRA059 & ALCA-1 & 11/28/2007 & 36995 & 3972 & 747 & 480 & 5186 & 41 & 14 & 133 & 73 & 13 & 97 & 11 \\
\hline KRA060 & ALCA-1 & 11/28/2007 & 40362 & 4193 & 734 & 480 & 5181 & 40 & 21 & 132 & 89 & 12 & 94 & 14 \\
\hline
\end{tabular}


Table DR2. ED-XRF concentration data

\begin{tabular}{|c|c|c|c|c|c|c|c|c|c|c|c|c|c|c|}
\hline ANID & Sub-source & Date & $\mathrm{K}(\mathrm{ppm})$ & $\mathrm{Ca}(\mathrm{ppm})$ & $\mathrm{Ti}(\mathrm{ppm})$ & $\mathrm{Mn}(\mathrm{ppm})$ & $\mathrm{Fe}(\mathrm{ppm})$ & $\mathrm{Zn}(\mathrm{ppm})$ & $\mathrm{Ga}(\mathrm{ppm})$ & $\mathrm{Rb}(\mathrm{ppm})$ & $\operatorname{Sr}(\mathrm{ppm})$ & $\mathrm{Y}(\mathrm{ppm})$ & $\operatorname{zr}(\mathrm{ppm})$ & $\mathrm{Nb}(\mathrm{ppm})$ \\
\hline KRA061 & ALCA-1 & 11/28/2007 & 38480 & 4333 & 752 & 483 & 5361 & 40 & 16 & 137 & 78 & 13 & 95 & 13 \\
\hline KRA062 & ALCA-6 & 11/28/2007 & 37697 & 3663 & 631 & 449 & 4977 & 35 & 21 & 121 & 76 & 10 & 89 & 13 \\
\hline KRA063 & ALCA-1 & 11/28/2007 & 37380 & 4042 & 678 & 487 & 5288 & 38 & 15 & 129 & 76 & 11 & 90 & 12 \\
\hline KRA064 & ALCA-1 & 11/28/2007 & 38202 & 4238 & 784 & 505 & 5459 & 40 & 19 & 132 & 86 & 13 & 95 & 13 \\
\hline KRA065 & ALCA-1 & $11 / 28 / 2007$ & 34415 & 3575 & 645 & 453 & 4919 & 36 & 15 & 129 & 72 & 11 & 87 & 11 \\
\hline KRA066 & ALCA-1 & 11/28/2007 & 35724 & 3760 & 683 & 469 & 5302 & 37 & 18 & 133 & 81 & 11 & 91 & 12 \\
\hline KRA067 & ALCA-1 & $11 / 28 / 2007$ & 35915 & 3883 & 750 & 466 & 5307 & 39 & 18 & 133 & 83 & 11 & 98 & 13 \\
\hline KRA068 & ALCA-1 & $11 / 28 / 2007$ & 40070 & 4336 & 768 & 507 & 5797 & 42 & 18 & 133 & 85 & 12 & 96 & 13 \\
\hline KRA069 & ALCA-1 & 11/28/2007 & 36308 & 4120 & 759 & 475 & 5271 & 39 & 19 & 132 & 87 & 13 & 97 & 13 \\
\hline KRA070 & ALCA-6 & 11/28/2007 & 32238 & 3122 & 678 & 418 & 4773 & 36 & 15 & 124 & 70 & 9 & 85 & 10 \\
\hline KRA071 & ALCA-1 & $11 / 28 / 2007$ & 34124 & 3471 & 681 & 455 & 5030 & 39 & 17 & 129 & 80 & 10 & 97 & 11 \\
\hline KRA072 & ALCA-1 & $11 / 28 / 2007$ & 35006 & 3682 & 730 & 442 & 4996 & 41 & 17 & 129 & 76 & 11 & 97 & 11 \\
\hline KRA073 & ALCA-1 & $11 / 28 / 2007$ & 33788 & 4059 & 729 & 465 & 5437 & 37 & 15 & 135 & 76 & 12 & 97 & 12 \\
\hline KRA074 & ALCA-1 & 11/28/2007 & 41363 & 4259 & 747 & 451 & 5250 & 43 & 17 & 129 & 77 & 12 & 98 & 13 \\
\hline KRA075 & ALCA-1 & $11 / 28 / 2007$ & 38804 & 4375 & 677 & 468 & 5069 & 40 & 14 & 132 & 74 & 14 & 97 & 12 \\
\hline KRA076 & ALCA-1 & $11 / 28 / 2007$ & 35071 & 4063 & 723 & 463 & 5312 & 39 & 17 & 134 & 82 & 12 & 102 & 12 \\
\hline KRA077 & ALCA-1 & 11/28/2007 & 34788 & 3264 & 676 & 449 & 4885 & 38 & 14 & 129 & 73 & 11 & 90 & 11 \\
\hline KRA078 & ALCA-1 & $11 / 28 / 2007$ & 43175 & 4902 & 818 & 491 & 5909 & 44 & 17 & 138 & 81 & 14 & 100 & 14 \\
\hline KRA079 & ALCA-1 & 11/28/2007 & 38495 & 4117 & 817 & 458 & 5307 & 43 & 20 & 141 & 89 & 13 & 104 & 14 \\
\hline KRA080 & ALCA-6 & 11/28/2007 & 31016 & 3435 & 599 & 450 & 4938 & 32 & 14 & 119 & 67 & 9 & 80 & 10 \\
\hline KRA081 & ALCA-1 & 11/28/2007 & 39889 & 4515 & 893 & 513 & 5633 & 41 & 23 & 137 & 98 & 14 & 99 & 15 \\
\hline KRA082 & ALCA-1 & 11/28/2007 & 37288 & 4205 & 782 & 489 & 5284 & 40 & 19 & 134 & 84 & 12 & 94 & 13 \\
\hline KRA083 & ALCA-1 & 11/28/2007 & 39060 & 4419 & 853 & 451 & 5467 & 42 & 23 & 135 & 96 & 12 & 99 & 15 \\
\hline KRA084 & ALCA-1 & $11 / 28 / 2007$ & 35909 & 3887 & 741 & 435 & 5038 & 38 & 16 & 131 & 80 & 14 & 93 & 12 \\
\hline KRA085 & ALCA-1 & 11/28/2007 & 37785 & 4250 & 824 & 451 & 5300 & 42 & 18 & 132 & 87 & 14 & 103 & 13 \\
\hline KRA086 & ALCA-1 & $11 / 28 / 2007$ & 33623 & 3652 & 700 & 440 & 4765 & 35 & 20 & 128 & 88 & 10 & 90 & 12 \\
\hline KRA087 & ALCA-1 & 11/28/2007 & 34747 & 3822 & 790 & 458 & 5204 & 40 & 22 & 131 & 93 & 14 & 100 & 13 \\
\hline KRA088 & ALCA-1 & 11/28/2007 & 36992 & 4091 & 733 & 441 & 5264 & 40 & 16 & 127 & 80 & 12 & 94 & 12 \\
\hline KRA089 & ALCA-1 & 11/28/2007 & 38126 & 4213 & 682 & 447 & 5359 & 38 & 15 & 127 & 74 & 12 & 89 & 12 \\
\hline KRA090 & ALCA-1 & 11/28/2007 & 35647 & 3879 & 669 & 431 & 5043 & 34 & 18 & 126 & 75 & 11 & 87 & 12 \\
\hline KRA091 & ALCA-6 & 11/29/2007 & 32588 & 3492 & 676 & 449 & 4790 & 35 & 20 & 125 & 80 & 10 & 86 & 12 \\
\hline KRA092 & ALCA-1 & 11/29/2007 & 34265 & 3715 & 774 & 473 & 5082 & 39 & 21 & 128 & 88 & 12 & 91 & 12 \\
\hline KRA093 & ALCA-1 & 11/29/2007 & 37706 & 4226 & 802 & 493 & 5401 & 40 & 17 & 135 & 84 & 14 & 99 & 13 \\
\hline KRA095 & ALCA-1 & 11/29/2007 & 37003 & 4056 & 729 & 449 & 5010 & 39 & 20 & 130 & 83 & 12 & 96 & 13 \\
\hline KRA096 & ALCA-1 & 11/29/2007 & 39088 & 4195 & 678 & 443 & 5237 & 36 & 20 & 133 & 78 & 13 & 89 & 14 \\
\hline KRA113 & ALCA-1 & 11/29/2007 & 36297 & 4030 & 704 & 475 & 5191 & 39 & 16 & 132 & 81 & 12 & 94 & 12 \\
\hline KRA114 & ALCA-1 & 9/23/2008 & 39752 & & 1146 & 424 & 5285 & 46 & 19 & 137 & 96 & 13 & 116 & 14 \\
\hline KRA115 & ALCA-1 & 9/23/2008 & 36726 & & 1150 & 332 & 5381 & 40 & 17 & 135 & 94 & 14 & 116 & 14 \\
\hline KRA116 & ALCA-1 & 9/23/2008 & 36093 & & 1084 & 384 & 5387 & 34 & 16 & 134 & 91 & 13 & 110 & 13 \\
\hline KRA117 & ALCA-1 & 9/23/2008 & 37659 & & 1101 & 363 & 5402 & 41 & 17 & 135 & 91 & 14 & 114 & 15 \\
\hline KRA118 & ALCA-1 & 9/23/2008 & 40653 & & 1153 & 376 & 5815 & 46 & 19 & 136 & 96 & 15 & 116 & 13 \\
\hline KRA119 & ALCA-1 & 9/23/2008 & 35868 & & 1135 & 394 & 5580 & 36 & 16 & 138 & 94 & 13 & 117 & 14 \\
\hline KRA120 & ALCA-1 & 9/23/2008 & 37627 & & 1093 & 378 & 5495 & 42 & 17 & 137 & 93 & 13 & 118 & 13 \\
\hline KRA121 & ALCA-1 & 9/23/2008 & 36243 & & 1133 & 366 & 5505 & 37 & 16 & 137 & 94 & 14 & 112 & 13 \\
\hline KRA122 & ALCA-1 & 9/23/2008 & 37623 & & 1127 & 324 & 5335 & 41 & 17 & 136 & 90 & 14 & 117 & 15 \\
\hline KRA123 & ALCA-1 & 9/23/2008 & 36517 & & 1139 & 356 & 5400 & 43 & 16 & 137 & 97 & 12 & 116 & 13 \\
\hline KRA126 & ALCA-1 & 9/23/2008 & 36145 & & 1144 & 373 & 5252 & 37 & 16 & 135 & 91 & 13 & 111 & 13 \\
\hline KRA127 & ALCA-1 & 9/23/2008 & 40999 & & 1185 & 371 & 5425 & 50 & 19 & 137 & 96 & 11 & 116 & 15 \\
\hline KRA128 & ALCA-1 & 9/23/2008 & 37773 & & 1120 & 380 & 5466 & 39 & 17 & 133 & 88 & 13 & 118 & 13 \\
\hline KRA129 & ALCA-1 & 9/23/2008 & 38877 & & 1141 & 383 & 5448 & 42 & 18 & 135 & 92 & 13 & 116 & 14 \\
\hline KRA130 & ALCA-1 & 9/23/2008 & 37541 & & 1070 & 351 & 5502 & 43 & 17 & 137 & 90 & 13 & 114 & 15 \\
\hline KRA131 & ALCA-1 & 9/23/2008 & 37475 & & 1101 & 397 & 5435 & 35 & 17 & 136 & 93 & 14 & 108 & 12 \\
\hline KRA132 & ALCA-1 & 9/23/2008 & 39128 & & 1121 & 377 & 5525 & 39 & 18 & 131 & 87 & 13 & 114 & 14 \\
\hline KRA133 & ALCA-1 & 9/23/2008 & 37031 & & 1088 & 375 & 5523 & 38 & 16 & 135 & 88 & 14 & 110 & 16 \\
\hline
\end{tabular}


Table DR2. ED-XRF concentration data

\begin{tabular}{|c|c|c|c|c|c|c|c|c|c|c|c|c|c|c|}
\hline ANID & Sub-source & Date & $\mathrm{K}(\mathrm{ppm})$ & $\mathrm{Ca}(\mathrm{ppm})$ & $\mathrm{Ti}(\mathrm{ppm})$ & $\mathrm{Mn}(\mathrm{ppm})$ & $\mathrm{Fe}(\mathrm{ppm})$ & $\mathrm{Zn}(\mathrm{ppm})$ & $\mathrm{Ga}(\mathrm{ppm})$ & $\mathrm{Rb}(\mathrm{ppm})$ & $\operatorname{Sr}(\mathrm{ppm})$ & $Y(p p m)$ & $\mathrm{Zr}(\mathrm{ppm})$ & $\mathrm{Nb}$ (ppm) \\
\hline KRA134 & ALCA-1 & 9/23/2008 & 39677 & & 1238 & 379 & 5658 & 38 & 19 & 140 & 93 & 16 & 115 & 13 \\
\hline KRA135 & ALCA-1 & 9/23/2008 & 37103 & & 1128 & 407 & 5372 & 41 & 16 & 137 & 93 & 14 & 117 & 15 \\
\hline KRA136 & ALCA-1 & 9/23/2008 & 38243 & & 1085 & 379 & 5269 & 43 & 17 & 134 & 88 & 12 & 116 & 16 \\
\hline KRA137 & ALCA-1 & 9/23/2008 & 35340 & & 1149 & 362 & 5671 & 37 & 15 & 126 & 94 & 13 & 113 & 13 \\
\hline KRA139 & ALCA-1 & 9/23/2008 & 38076 & & 1105 & 360 & 5477 & 39 & 17 & 137 & 90 & 15 & 114 & 14 \\
\hline KRA140 & ALCA-1. & 9/23/2008 & 38012 & & 1154 & 388 & 5445 & 42 & 17 & 139 & 97 & 13 & 116 & 13 \\
\hline KRA141 & ALCA-1 & $9 / 23 / 2008$ & 40583 & & 1070 & 383 & 5435 & 48 & 19 & 137 & 90 & 14 & 115 & 14 \\
\hline KRA142 & ALCA-1. & $9 / 23 / 2008$ & 37631 & & 1126 & 402 & 5519 & 38 & 17 & 134 & 96 & 12 & 116 & 13 \\
\hline KRA143 & ALCA-1 & 9/23/2008 & 39880 & & 1109 & 368 & 5466 & 46 & 18 & 138 & 94 & 12 & 114 & 17 \\
\hline KRA144 & ALCA-1 & 9/23/2008 & 37854 & & 1137 & 412 & 5545 & 36 & 17 & 137 & 91 & 14 & 117 & 13 \\
\hline KRA145 & ALCA-1 & 9/23/2008 & 38339 & & 1109 & 382 & 5403 & 48 & 18 & 138 & 95 & 13 & 119 & 14 \\
\hline KRA146 & ALCA-1 & $9 / 23 / 2008$ & 41619 & & 1124 & 447 & 5519 & 47 & 19 & 142 & 92 & 12 & 111 & 17 \\
\hline KRA149 & ALCA-1 & 9/23/2008 & 36224 & & 1082 & 367 & 5618 & 39 & 16 & 137 & 95 & 13 & 117 & 12 \\
\hline KRA150 & ALCA-1 & 9/23/2008 & 38670 & & 1075 & 402 & 5680 & 40 & 17 & 138 & 96 & 13 & 114 & 13 \\
\hline KRA152 & ALCA-1 & 9/24/2008 & 37847 & & 1118 & 306 & 5469 & 37 & 17 & 135 & 94 & 13 & 114 & 13 \\
\hline KRA153 & ALCA-1 & 9/24/2008 & 37890 & & 1092 & 355 & 5479 & 39 & 17 & 136 & 89 & 14 & 117 & 13 \\
\hline KRA175 & ALCA-1 & $9 / 24 / 2008$ & 37470 & & 1195 & 483 & 5645 & 41 & 17 & 140 & 97 & 13 & 118 & 14 \\
\hline KRA196 & ALCA-1 & 9/30/2008 & 36285 & & 1121 & 296 & 5398 & 33 & 16 & 135 & 87 & 13 & 108 & 13 \\
\hline KRA226 & ALCA-1 & $1 / 21 / 2009$ & 34331 & & 713 & 440 & 5535 & 36 & 13 & 136 & 95 & 14 & 110 & 18 \\
\hline KRA228 & ALCA-1 & $1 / 20 / 2009$ & 35524 & & 798 & 387 & 5782 & 40 & 14 & 140 & 101 & 16 & 113 & 20 \\
\hline KRA230 & ALCA-1 & 1/20/2009 & 37794 & & 855 & 214 & 5944 & 35 & 15 & 132 & 98 & 10 & 96 & 9 \\
\hline KRA236 & ALCA-1 & $1 / 20 / 2009$ & 35120 & & 800 & 271 & 5614 & 33 & 13 & 132 & 96 & 8 & 90 & 10 \\
\hline KRA237 & ALCA-1 & $1 / 20 / 2009$ & 36240 & & 781 & 462 & 5613 & 40 & 15 & 136 & 95 & 14 & 114 & 21 \\
\hline KRA239 & ALCA-1 & $1 / 20 / 2009$ & 34478 & & 763 & 407 & 5688 & 39 & 13 & 135 & 100 & 15 & 111 & 23 \\
\hline KRA241 & ALCA-1 & $1 / 20 / 2009$ & 39664 & & 748 & 221 & 6039 & 35 & 16 & 131 & 94 & 9 & 92 & 11 \\
\hline KRA003 & ALCA-2 & 11/20/2007 & 37658 & 5495 & 1154 & 441 & 7117 & 43 & 16 & 134 & 142 & 17 & 141 & 14 \\
\hline KRA004 & ALCA-2 & $11 / 20 / 2007$ & 38347 & 5507 & 1147 & 430 & 6945 & 40 & 14 & 138 & 153 & 19 & 137 & 14 \\
\hline KRA151 & ALCA-2 & 9/24/2008 & 37698 & & 1286 & 293 & 7048 & 46 & 16 & 144 & 156 & 13 & 156 & 12 \\
\hline KRA010 & ALCA-3 & $11 / 20 / 2007$ & 33407 & 6198 & 1310 & 484 & 7731 & 43 & 13 & 121 & 199 & 18 & 148 & 12 \\
\hline KRA011 & ALCA-3 & $11 / 20 / 2007$ & 34497 & 6502 & 1280 & 471 & 7816 & 43 & 14 & 121 & 195 & 17 & 152 & 12 \\
\hline KRA012 & ALCA-3 & $11 / 20 / 2007$ & 32075 & 5931 & 1316 & 466 & 7388 & 37 & 21 & 119 & 238 & 16 & 140 & 14 \\
\hline KRA013 & ALCA-3 & $11 / 20 / 2007$ & 34693 & 6556 & 1321 & 484 & 7694 & 40 & 17 & 122 & 215 & 16 & 148 & 13 \\
\hline KRA094 & ALCA-3 & $11 / 29 / 2007$ & 36183 & 7145 & 1562 & 484 & 7786 & 43 & 24 & 121 & 262 & 19 & 151 & 15 \\
\hline KRA124 & ALCA-3 & 9/23/2008 & 34874 & & 1319 & 388 & 8122 & 44 & 14 & 131 & 239 & 14 & 162 & 11 \\
\hline KRA125 & ALCA-3 & 9/23/2008 & 39563 & & 1266 & 426 & 8379 & 57 & 15 & 129 & 237 & 14 & 160 & 12 \\
\hline KRA138 & ALCA-3 & 9/23/2008 & 35809 & & 1671 & 263 & 9163 & 49 & 14 & 140 & 270 & 16 & 205 & 13 \\
\hline KRA147 & ALCA-3 & 9/23/2008 & 38901 & & 1361 & 398 & 8215 & 55 & 15 & 130 & 237 & 13 & 157 & 13 \\
\hline KRA148 & ALCA-3 & 9/23/2008 & 36387 & & 1239 & 305 & 7900 & 42 & 15 & 127 & 235 & 14 & 153 & 10 \\
\hline KRA154 & ALCA-3 & 9/24/2008 & 38006 & & 1524 & 318 & 8607 & 51 & 15 & 137 & 203 & 12 & 180 & 14 \\
\hline KRA155 & ALCA-3 & $9 / 24 / 2008$ & 37860 & & 1377 & 370 & 8136 & 50 & 15 & 131 & 236 & 15 & 155 & 12 \\
\hline KRA156 & ALCA-3 & 9/24/2008 & 36497 & & 1396 & 418 & 7853 & 50 & 14 & 123 & 233 & 15 & 150 & 12 \\
\hline KRA157 & ALCA-3 & 9/24/2008 & 36653 & & 1388 & 324 & 8241 & 43 & 15 & 132 & 240 & 14 & 159 & 11 \\
\hline KRA158 & ALCA-3 & $9 / 24 / 2008$ & 35608 & & 1305 & 341 & 7849 & 47 & 14 & 125 & 230 & 13 & 158 & 10 \\
\hline KRA159 & ALCA-3 & 9/24/2008 & 34570 & & 1417 & 387 & 8085 & 44 & 14 & 132 & 237 & 13 & 161 & 12 \\
\hline KRA160 & ALCA-3 & $9 / 24 / 2008$ & 38946 & & 1400 & 397 & 8390 & 56 & 16 & 133 & 250 & 16 & 163 & 13 \\
\hline KRA161 & ALCA-3 & 9/24/2008 & 37385 & & 1436 & 309 & 8172 & 57 & 14 & 129 & 238 & 13 & 161 & 14 \\
\hline KRA162 & ALCA-3 & 9/24/2008 & 38152 & & 1361 & 417 & 8199 & 53 & 15 & 131 & 238 & 14 & 162 & 13 \\
\hline KRA163 & ALCA-3 & $9 / 24 / 2008$ & 36313 & & 1295 & 309 & 8063 & 44 & 15 & 128 & 234 & 15 & 155 & 11 \\
\hline KRA164 & ALCA-3 & $9 / 24 / 2008$ & 38876 & & 1395 & 382 & 8297 & 57 & 15 & 133 & 249 & 13 & 162 & 13 \\
\hline KRA165 & ALCA-3 & 9/24/2008 & 33513 & & 1341 & 377 & 8094 & 40 & 13 & 130 & 238 & 13 & 155 & 11 \\
\hline KRA166 & ALCA-3 & 9/24/2008 & 37325 & & 1439 & 412 & 8215 & 54 & 15 & 131 & 241 & 16 & 161 & 13 \\
\hline KRA167 & ALCA-3 3 & 9/24/2008 & 35073 & & 1410 & 348 & 8324 & 50 & 14 & 130 & 245 & 14 & 167 & 11 \\
\hline
\end{tabular}


Table DR2. ED-XRF concentration data

\begin{tabular}{|c|c|c|c|c|c|c|c|c|c|c|c|c|c|c|}
\hline ANID & Sub-source & Date & $\mathrm{K}(\mathrm{ppm})$ & $\mathrm{Ca}(\mathrm{ppm})$ & $\mathrm{Ti}(\mathrm{ppm})$ & $\mathrm{Mn}(\mathrm{ppm})$ & $\mathrm{Fe}(\mathrm{ppm})$ & $\mathrm{Zn}(\mathrm{ppm})$ & $\mathrm{Ga}(\mathrm{ppm})$ & $\mathrm{Rb}(\mathrm{ppm})$ & $\operatorname{Sr}(\mathrm{ppm})$ & $Y(p p m)$ & $\mathrm{Zr}(\mathrm{ppm})$ & $\mathrm{Nb}$ (ppm) \\
\hline KRA168 & ALCA-3 & 9/24/2008 & 36164 & & 1435 & 425 & 8229 & 46 & 15 & 132 & 237 & 14 & 159 & 11 \\
\hline KRA169 & ALCA-3 & 9/24/2008 & 35760 & & 1299 & 370 & 8076 & 44 & 14 & 130 & 237 & 15 & 159 & 11 \\
\hline KRA170 & ALCA-3 & $9 / 24 / 2008$ & 37672 & & 1472 & 420 & 8293 & 51 & 15 & 134 & 249 & 14 & 162 & 12 \\
\hline KRA171 & ALCA-3 & $9 / 24 / 2008$ & 35985 & & 1450 & 392 & 8172 & 45 & 15 & 132 & 240 & 16 & 160 & 14 \\
\hline KRA172 & ALCA-3 & 9/24/2008 & 36091 & & 1438 & 353 & 8345 & 50 & 14 & 135 & 246 & 15 & 167 & 13 \\
\hline KRA173 & ALCA-3 & 9/24/2008 & 36738 & & 1338 & 385 & 8208 & 47 & 15 & 130 & 238 & 14 & 160 & 11 \\
\hline KRA097 & ALCA-4 & 11/29/2007 & 28994 & 4461 & 1016 & 503 & 5401 & 32 & 25 & 99 & 180 & 10 & 90 & 11 \\
\hline KRA098 & ALCA-4 & $11 / 29 / 2007$ & 29944 & 4603 & 954 & 505 & 5408 & 32 & 17 & 100 & 151 & 12 & 89 & 10 \\
\hline KRA099 & ALCA-4 & 11/29/2007 & 31032 & 4614 & 921 & 518 & 5556 & 33 & 20 & 102 & 163 & 13 & 93 & 11 \\
\hline KRA100 & ALCA-4 & 11/29/2007 & 31381 & 5034 & 937 & 501 & 5504 & 32 & 19 & 101 & 171 & 14 & 90 & 11 \\
\hline KRA103 & ALCA-4 & 11/29/2007 & 31927 & 4899 & 899 & 517 & 5985 & 32 & 18 & 101 & 147 & 12 & 85 & 11 \\
\hline KRA221 & ALCA-4 & 9/30/2008 & 31656 & & 1150 & 446 & 5635 & 33 & 13 & 107 & 163 & 12 & 106 & 10 \\
\hline KRA242 & ALCA-4 & 1/20/2009 & 30639 & & 804 & 549 & 5515 & 31 & 11 & 98 & 177 & 7 & 88 & 13 \\
\hline KRA253 & ALCA-4 & 1/21/2009 & 29961 & & 783 & 703 & 6023 & 36 & 12 & 106 & 163 & 13 & 108 & 15 \\
\hline KRA254 & ALCA-4 & 1/21/2009 & 30971 & & 863 & 585 & 5560 & 35 & 12 & 105 & 166 & 10 & 101 & 14 \\
\hline KRA255 & ALCA-4 & 1/21/2009 & 31990 & & 794 & 549 & 5582 & 31 & 13 & 103 & 187 & 11 & 101 & 16 \\
\hline KRA256 & ALCA-4 & 1/21/2009 & 32677 & & 522 & 233 & 5341 & 34 & 13 & 105 & 185 & 7 & 82 & 7 \\
\hline KRA257 & ALCA-4 & 1/21/2009 & 31877 & & 772 & 359 & 5443 & 34 & 12 & 103 & 179 & 8 & 91 & 11 \\
\hline KRA258 & ALCA-4 & 1/20/2009 & 30446 & & 796 & 720 & 5719 & 33 & 12 & 105 & 168 & 11 & 107 & 18 \\
\hline KRA104 & ALCA- 5 & 11/29/2007 & 34962 & 4409 & 877 & 453 & 5291 & 37 & 19 & 123 & 134 & 13 & 99 & 12 \\
\hline KRA105 & ALCA- 5 & 11/29/2007 & 33894 & 4253 & 860 & 457 & 5120 & 39 & 16 & 119 & 116 & 14 & 95 & 11 \\
\hline KRA106 & ALCA-5 & $11 / 29 / 2007$ & 34721 & 3974 & 838 & 441 & 4962 & 38 & 20 & 118 & 122 & 11 & 86 & 12 \\
\hline KRA107 & ALCA- 5 & 11/29/2007 & 35498 & 4143 & 839 & 474 & 4993 & 40 & 18 & 124 & 114 & 12 & 89 & 12 \\
\hline KRA108 & ALCA -5 & 11/29/2007 & 37668 & 4557 & 782 & 460 & 5040 & 38 & 18 & 124 & 117 & 13 & 91 & 13 \\
\hline KRA109 & ALCA- 5 & 11/29/2007 & 38715 & 4336 & 836 & 450 & 4986 & 41 & 17 & 128 & 117 & 15 & 89 & 12 \\
\hline KRA110 & ALCA- 5 & 11/29/2007 & 37836 & 4216 & 764 & 459 & 4952 & 38 & 18 & 126 & 111 & 14 & 88 & 12 \\
\hline KRA111 & ALCA- 5 & 11/29/2007 & 36685 & 4214 & 770 & 485 & 4951 & 38 & 14 & 124 & 99 & 13 & 87 & 11 \\
\hline KRA112 & ALCA- 5 & 11/29/2007 & 35636 & 4340 & 709 & 465 & 5019 & 35 & 13 & 124 & 101 & 16 & 83 & 11 \\
\hline KRA174 & ALCA- 5 & 9/24/2008 & 37417 & & 1221 & 383 & 5765 & 46 & 16 & 126 & 137 & 13 & 114 & 14 \\
\hline KRA176 & ALCA -5 & 9/24/2008 & 33836 & & 1071 & 263 & 4372 & 31 & 14 & 134 & 130 & 12 & 90 & 14 \\
\hline KRA190 & ALCA -5 & 9/30/2008 & 38601 & & 1074 & 387 & 5184 & 46 & 17 & 132 & 125 & 13 & 105 & 15 \\
\hline KRA209 & ALCA-5 & 9/30/2008 & 42234 & & 1163 & 437 & 6037 & 59 & 19 & 144 & 155 & 14 & 121 & 16 \\
\hline KRA210 & ALCA- 5 & 9/30/2008 & 39736 & & 1163 & 357 & 5502 & 42 & 18 & 131 & 137 & 12 & 112 & 14 \\
\hline KRA211 & ALCA- 5 & 9/30/2008 & 38201 & & 1127 & 411 & 5249 & 36 & 17 & 135 & 126 & 14 & 103 & 13 \\
\hline KRA212 & ALCA- 5 & 9/30/2008 & 38060 & & 1143 & 418 & 5179 & 44 & 17 & 131 & 130 & 12 & 108 & 13 \\
\hline KRA213 & ALCA- 5 & 9/30/2008 & 35710 & & 1063 & 411 & 5188 & 40 & 15 & 128 & 130 & 13 & 104 & 12 \\
\hline KRA214 & ALCA- 5 & 9/30/2008 & 36708 & & 1127 & 399 & 5010 & 44 & 17 & 132 & 132 & 12 & 109 & 12 \\
\hline KRA215 & ALCA- 5 & 9/30/2008 & 39940 & & 1154 & 434 & 5352 & 44 & 18 & 136 & 135 & 13 & 106 & 14 \\
\hline KRA217 & ALCA- 5 & 9/30/2008 & 36173 & & 1161 & 417 & 5070 & 41 & 16 & 133 & 131 & 13 & 109 & 13 \\
\hline KRA218 & ALCA- 5 & 9/30/2008 & 42173 & & 1105 & 414 & 5141 & 51 & 20 & 131 & 129 & 12 & 106 & 12 \\
\hline KRA219 & ALCA- 5 & 9/30/2008 & 38533 & & 1107 & 378 & 5247 & 47 & 17 & 132 & 129 & 13 & 106 & 15 \\
\hline KRA220 & ALCA- 5 & 9/30/2008 & 34052 & & 1194 & 433 & 5195 & 41 & 14 & 126 & 130 & 13 & 108 & 16 \\
\hline KRA222 & ALCA- 5 & 1/20/2009 & 35403 & & 857 & 475 & 5315 & 37 & 14 & 129 & 134 & 11 & 89 & 19 \\
\hline KRA223 & ALCA- 5 & 1/20/2009 & 33423 & & 717 & 459 & 5204 & 36 & 13 & 131 & 128 & 13 & 109 & 18 \\
\hline KRA224 & ALCA- 5 & 1/20/2009 & 37190 & & 818 & 425 & 5283 & 36 & 15 & 129 & 127 & 14 & 95 & 19 \\
\hline KRA225 & ALCA- 5 & 1/21/2009 & 34406 & & 774 & 408 & 5316 & 33 & 14 & 124 & 144 & 14 & 94 & 14 \\
\hline KRA227 & ALCA- 5 & 1/21/2009 & 33359 & & 781 & 287 & 5186 & 32 & 12 & 128 & 141 & 10 & 84 & 8 \\
\hline KRA229 & ALCA- 5 & 1/20/2009 & 35221 & & 839 & 352 & 5483 & 37 & 14 & 133 & 135 & 12 & 90 & 16 \\
\hline KRA231 & ALCA- 5 & 1/20/2009 & 34433 & & 800 & 509 & 5653 & 46 & 14 & 129 & 142 & 11 & 104 & 20 \\
\hline KRA232 & ALCA- 5 & 1/21/2009 & 34395 & & 766 & 337 & 5349 & 33 & 13 & 126 & 124 & 10 & 83 & 10 \\
\hline KRA233 & ALCA- 5 & 1/20/2009 & 32510 & & 856 & 456 & 5341 & 34 & 12 & 123 & 139 & 12 & 97 & 18 \\
\hline KRA234 & ALCA- 5 & 1/20/2009 & 35522 & & 733 & 552 & 5323 & 41 & 15 & 129 & 127 & 13 & 97 & 23 \\
\hline
\end{tabular}


Table DR2. ED-XRF concentration data

\begin{tabular}{|c|c|c|c|c|c|c|c|c|c|c|c|c|c|c|}
\hline ANID & Sub-source & Date & $K(p p m)$ & $\mathrm{Ca}(\mathrm{ppm})$ & $\mathrm{Ti}(\mathrm{ppm})$ & $\mathrm{Mn}(\mathrm{ppm})$ & $\mathrm{Fe}(\mathrm{ppm})$ & $\mathrm{Zn}(\mathrm{ppm})$ & $\mathrm{Ga}(\mathrm{ppm})$ & $\mathrm{Rb}(\mathrm{ppm})$ & $\operatorname{Sr}(\mathrm{ppm})$ & $Y(p p m)$ & $\mathrm{zr}(\mathrm{ppm})$ & $\mathrm{Nb}(\mathrm{ppm})$ \\
\hline KRA235 & ALCA -5 & 1/20/2009 & 34224 & & 798 & 417 & 5637 & 41 & 14 & 126 & 141 & 11 & 96 & 14 \\
\hline KRA240 & ALCA-5 & 1/20/2009 & 33737 & & 823 & 450 & 5106 & 34 & 13 & 128 & 131 & 12 & 95 & 16 \\
\hline KRA244 & ALCA- 5 & 1/21/2009 & 34805 & & 790 & 381 & 5637 & 37 & 14 & 125 & 142 & 10 & 97 & 10 \\
\hline KRA246 & ALCA-5 & 1/21/2009 & 34532 & & 749 & 380 & 5284 & 34 & 13 & 133 & 136 & 10 & 90 & 15 \\
\hline KRA247 & ALCA -5 & 1/20/2009 & 34888 & & 807 & 512 & 5181 & 37 & 14 & 128 & 129 & 15 & 96 & 23 \\
\hline KRA248 & ALCA-5 & 1/21/2009 & 36628 & & 756 & 478 & 5358 & 35 & 16 & 128 & 131 & 14 & 95 & 18 \\
\hline KRA249 & ALCA -5 & 1/21/2009 & 35830 & & 657 & 358 & 5242 & 40 & 15 & 130 & 135 & 14 & 92 & 15 \\
\hline KRA250 & ALCA-5 & 1/21/2009 & 37509 & & 860 & 491 & 5281 & 36 & 16 & 127 & 131 & 15 & 98 & 22 \\
\hline KRA251 & ALCA-5 & 1/21/2009 & 34234 & & 822 & 308 & 5209 & 33 & 13 & 132 & 137 & 9 & 84 & 14 \\
\hline KRA101 & ALCA-7 & 11/29/2007 & 34722 & 4942 & 741 & 462 & 4870 & 28 & 20 & 150 & 151 & 18 & 79 & 14 \\
\hline KRA102 & ALCA-7 & 11/29/2007 & 37418 & 5115 & 772 & 436 & 4836 & 33 & 18 & 150 & 145 & 17 & 83 & 14 \\
\hline KRA177 & ALCA-7 & 9/24/2008 & 37668 & & 1069 & 331 & 5232 & 29 & 17 & 160 & 143 & 15 & 94 & 11 \\
\hline KRA178 & ALCA -7 & 9/24/2008 & 38481 & & 1130 & 355 & 4976 & 39 & 18 & 161 & 151 & 15 & 101 & 13 \\
\hline KRA179 & ALCA-7 & 9/24/2008 & 39261 & & 1089 & 375 & 5089 & 33 & 18 & 159 & 147 & 15 & 96 & 12 \\
\hline KRA180 & ALCA -7 & 9/24/2008 & 41438 & & 1091 & 352 & 5267 & 42 & 19 & 164 & 155 & 15 & 100 & 14 \\
\hline KRA181 & ALCA-7 & 9/24/2008 & 36539 & & 1111 & 347 & 5073 & 28 & 16 & 162 & 150 & 15 & 96 & 12 \\
\hline KRA182 & ALCA-7 & 9/24/2008 & 37491 & & 1111 & 342 & 5078 & 36 & 17 & 157 & 149 & 15 & 101 & 14 \\
\hline KRA183 & ALCA-7 & 9/24/2008 & 38497 & & 1117 & 345 & 5010 & 37 & 17 & 158 & 146 & 16 & 96 & 16 \\
\hline KRA184 & ALCA-7 & 9/24/2008 & 39420 & & 1143 & 332 & 4944 & 36 & 17 & 157 & 148 & 13 & 96 & 17 \\
\hline KRA185 & ALCA-7 & 9/24/2008 & 38273 & & 1096 & 357 & 4918 & 31 & 17 & 160 & 147 & 16 & 95 & 12 \\
\hline KRA186 & ALCA-7 & 9/24/2008 & 37836 & & 1092 & 340 & 5067 & 43 & 17 & 150 & 150 & 15 & 100 & 11 \\
\hline KRA187 & ALCA-7 & 9/24/2008 & 36881 & & 1123 & 351 & 5013 & 28 & 16 & 157 & 150 & 15 & 95 & 12 \\
\hline KRA188 & ALCA-7 & 9/30/2008 & 35337 & & 1087 & 329 & 5004 & 28 & 15 & 156 & 149 & 15 & 97 & 11 \\
\hline KRA189 & ALCA-7 & 9/30/2008 & 39179 & & 1065 & 265 & 5105 & 37 & 17 & 155 & 147 & 15 & 97 & 14 \\
\hline KRA191 & ALCA-7 & 9/30/2008 & 36471 & & 1074 & 308 & 4949 & 31 & 16 & 157 & 143 & 14 & 95 & 11 \\
\hline KRA192 & ALCA-7 & 9/30/2008 & 37331 & & 1103 & 332 & 4887 & 28 & 17 & 160 & 146 & 17 & 94 & 12 \\
\hline KRA193 & ALCA-7 & 9/30/2008 & 38429 & & 1102 & 377 & 5016 & 42 & 17 & 158 & 156 & 14 & 105 & 13 \\
\hline KRA195 & ALCA-7 & 9/30/2008 & 37040 & & 1136 & 362 & 5049 & 36 & 16 & 159 & 154 & 14 & 100 & 15 \\
\hline KRA197 & ALCA-7 & 9/30/2008 & 37127 & & 1096 & 360 & 5097 & 33 & 16 & 159 & 149 & 14 & 96 & 14 \\
\hline KRA198 & ALCA-7 & 9/30/2008 & 39534 & & 1066 & 321 & 4959 & 32 & 18 & 159 & 149 & 18 & 95 & 11 \\
\hline KRA199 & ALCA-7 & 9/30/2008 & 38822 & & 1218 & 272 & 5598 & 34 & 17 & 169 & 158 & 15 & 99 & 15 \\
\hline KRA200 & ALCA-7 & 9/30/2008 & 34369 & & 1086 & 318 & 5078 & 24 & 15 & 157 & 145 & 15 & 92 & 11 \\
\hline KRA201 & ALCA-7 & 9/30/2008 & 37378 & & 1116 & 320 & 5100 & 31 & 17 & 158 & 150 & 14 & 98 & 12 \\
\hline KRA202 & ALCA-7 & 9/30/2008 & 34976 & & 1263 & 340 & 5523 & 31 & 15 & 167 & 160 & 17 & 105 & 14 \\
\hline KRA203 & ALCA -7 & 9/30/2008 & 37452 & & 1120 & 338 & 5175 & 27 & 17 & 166 & 156 & 13 & 97 & 12 \\
\hline KRA204 & ALCA-7 & 9/30/2008 & 35309 & & 1193 & 311 & 5460 & 32 & 15 & 156 & 156 & 15 & 100 & 12 \\
\hline KRA205 & ALCA -7 & 9/30/2008 & 38322 & & 1105 & 324 & 5035 & 41 & 17 & 160 & 148 & 14 & 101 & 16 \\
\hline KRA207 & ALCA-7 & 9/30/2008 & 37247 & & 1141 & 339 & 4978 & 32 & 16 & 160 & 148 & 15 & 97 & 13 \\
\hline KRA238 & ALCA-7 & 1/20/2009 & 33174 & & 690 & 507 & 4944 & 31 & 14 & 155 & 164 & 18 & 103 & 19 \\
\hline KRA243 & ALCA-7 & 1/21/2009 & 35406 & & 675 & 366 & 5233 & 28 & 15 & 159 & 169 & 13 & 88 & 13 \\
\hline
\end{tabular}


Table DR3. Alca obsidian NAA concentration data

\begin{tabular}{|c|c|c|c|c|c|c|c|c|c|}
\hline SANID & Sub-source & Short_rdf & Short Date & Al (ppm) & $\mathrm{Cl}(\mathrm{ppm})$ & Dy (ppm) & $K(\mathrm{ppm})$ & $\mathrm{Mn}(\mathrm{ppm})$ & $\mathrm{Na}$ (ppm) \\
\hline KRA001 & ALCA-1 & RAM1-S & 1/177/2008 & 68844 & 517 & 1.92 & 33352 & 467 & 30770 \\
\hline KRAO02 & ALCA-1 & RAM1-S & 1/17//2008 & 70101 & 609 & 1.42 & 38394 & 477 & 31500 \\
\hline KRA095 & ALCA-1 & CGR1-S & 2/16/2008 & 76561 & 460 & 2.04 & 36386 & 486 & 31825 \\
\hline A038 & ALCA-6 & RAM1-S & 17/2008 & 71582 & 446 & 2.41 & 37644 & 478 & 31609 \\
\hline KRA041 & ALCA-6 & RAM1-S & $1 / 17 / 2008$ & 68659 & 494 & 1.92 & 34049 & 466 & 30776 \\
\hline KRA046 & ALCA-6 & RAM1-S & 1/177/2008 & 73438 & 593 & 2.18 & 39705 & 469 & 29662 \\
\hline KRA062 & ALCA-6 & RAM1-S & 1/17/2008 & 74240 & 488 & 2.03 & 39260 & 479 & 29382 \\
\hline KRA070 & ALCA-6 & RAM1-S & 1/17/2008 & 66509 & 645 & 1.75 & 33172 & 471 & 30939 \\
\hline KRA080 & ALCA-6 & RAM1-S & 1/177/2008 & 69709 & 606 & 1.99 & 36389 & 462 & 30840 \\
\hline KRA091 & ALCA-6 & RAM1-S & 1/17/2008 & 73131 & 563 & 2.25 & 35896 & 471 & 30698 \\
\hline A003 & ALCA-2 & RAM1 & $7 / 26$ & 75738 & 612 & 2.04 & 33020 & 445 & 32368 \\
\hline KRAO04 & ALCA-2 & RAM1-S & 1/17//2008 & 81677 & 456 & 1.94 & 33403 & 442 & 33637 \\
\hline KRA010 & ALCA-3 & RAM1-S & 1/17//2008 & 70236 & 549 & 2.36 & 34424 & 559 & 32394 \\
\hline KRA011 & ALCA-3 & RAM1-S & 1/17//2008 & 76144 & 643 & 1.65 & 31618 & 559 & 32645 \\
\hline KRA012 & ALCA-3 & RAM1-S & 1/17/2008 & 77933 & 607 & 1.76 & 31768 & 570 & 32964 \\
\hline KRA013 & ALCA-3 & RAM1-S & 1/17/2008 & 74456 & 594 & 2.28 & 30949 & 554 & 31631 \\
\hline KRA094 & ALCA-3 & RAM1-S & $1 / 17 / 2008$ & 79193 & 601 & 2.22 & 34984 & 564 & 32740 \\
\hline KRA097 & ALCA-4 & RAM1-S & 1/17/2008 & 71006 & 448 & 1.42 & 28831 & 553 & 31839 \\
\hline KRA098 & ALCA- 4 & RAM1-S & 1/17//2008 & 68686 & 343 & 1.73 & 31799 & 556 & 32141 \\
\hline KRA099 & ALCA-4 & RAM1-S & 1/17/2008 & 72710 & 362 & 1.97 & 31604 & 554 & 31653 \\
\hline KRA100 & ALCA-4 & RAM1-S & 1/17/2008 & 71478 & 487 & 1.51 & 29571 & 549 & 31687 \\
\hline KRA103 & ALCA-4 & RAM1-S & 1/17//2008 & 72882 & 382 & 1.53 & 30827 & 550 & 31612 \\
\hline KRA10 & ALCA-5 & RAM1 & 1117 & 7014 & 551 & 2.01 & 3534 & 483 & 31948 \\
\hline KRA105 & ALCA-5 & RAM1-S & $1 / 17 / 2$ & 73258 & 554 & 2.08 & 34790 & 473 & 31460 \\
\hline KRA106 & ALC & RAM & & 73364 & 597 & 1.84 & 36951 & 474 & 30310 \\
\hline KRA107 & ALCA-5 & RAM1-S & $1 / 17 / 2$ & 72223 & 611 & 1.76 & 35454 & 479 & 31651 \\
\hline KRA108 & ALCA-5 & RAM1-S & 1/17/2008 & 68747 & 610 & 1.92 & 32738 & 476 & 30801 \\
\hline KRA109 & ALCA-5 & CGR1-S & 2/16/2008 & 71277 & 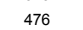 & 1. & 3243 & 484 & 31521 \\
\hline KRA110 & ALCA & CGR & $2 / 16 / 2$ & 735 & 412 & 2.0 & 365 & 482 & 31102 \\
\hline KRA111 & ALCA & CGR & & 736 & 48 & 2.2 & 359 & $48 \quad-$ & 31503 \\
\hline KRA112 & ALCA-5 & CGR1-S & $2 / 16 / 2008$ & 73436 & 546 & 2.11 & 34706 & 483 & 32321 \\
\hline A101 & ALCA & RAM1. & & 655 & 414 & 1.88 & 3545 & 448 & 28583 \\
\hline KRA102 & ALCA-7 & RAM1-S & 1/17/2008 & 66516 & 414 & 2.35 & 31105 & 446 & 29144 \\
\hline
\end{tabular}




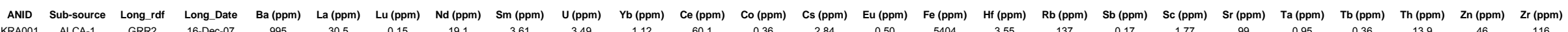

\begin{tabular}{|c|c|c|c|c|c|c|c|c|c|c|c|c|c|c|c|c|c|c|c|c|c|c|c|c|c|}
\hline KRA001 & ALCA-1 & GRR2 & 16-Dec-07 & 995 & 30.5 & 0.15 & 19.1 & 3.61 & 3.49 & 1.12 & 60.1 & 0.36 & 2.84 & 0.50 & 5404 & 3.55 & 137 & 0.17 & 1.77 & 99 & 0.95 & 0.36 & 13.9 & 46 & 116 \\
\hline KRA002 & ALCA-1 & GRR2 & 16-Dec-07 & 989 & 28.4 & 0.16 & 19.7 & 3.56 & 3.33 & 1.09 & 57.1 & 0.36 & 2.82 & 0.49 & 5375 & 3.52 & 136 & 0.15 & 1.77 & 98 & 0.95 & 0.35 & 13.8 & 45 & 109 \\
\hline KRA095 & ALCA-1 & GRR2 & 16-Dec-07 & 1022 & 31.2 & 0.15 & 20.6 & 3.64 & 3.30 & 1.15 & 62.1 & 0.24 & 2.85 & 0.50 & 5501 & 3.60 & 140 & 0.17 & 1.81 & 88 & 0.96 & 0.38 & 14.2 & 47 & 119 \\
\hline KRA038 & ALCA- 6 & GRR2 & 16-Dec-07 & 988 & 28.4 & 0.14 & 18.2 & 3.52 & 3.51 & 1.06 & 57.3 & 0.23 & 2.79 & 0.50 & 5402 & 3.54 & 137 & 0.16 & 1.77 & 91 & 0.96 & 0.36 & 13.8 & 41 & 109 \\
\hline KRA041 & ALCA- 6 & GRR2 & 16-Dec-07 & 1010 & 28.4 & 0.15 & 18.6 & 3.56 & 3.31 & 1.15 & 57.9 & 0.23 & 2.81 & 0.50 & 5438 & 3.55 & 138 & 0.16 & 1.78 & 106 & 0.95 & 0.36 & 13.8 & 41 & 122 \\
\hline KRA046 & ALCA- 6 & GRR2 & 16-Dec-07 & 999 & 29.1 & 0.16 & 19.0 & 3.58 & 3.28 & 1.14 & 58.3 & 0.22 & 2.80 & 0.49 & 5409 & 3.60 & 137 & 0.17 & 1.78 & 84 & 0.94 & 0.35 & 13.9 & 41 & 111 \\
\hline KRA062 & ALCA- 6 & GRR2 & 16-Dec-07 & 1005 & 28.7 & 0.16 & 18.9 & 3.55 & 3.50 & 1.10 & 57.6 & 0.36 & 2.82 & 0.50 & 5450 & 3.71 & 143 & 0.17 & 1.79 & 89 & 0.95 & 0.35 & 13.9 & 41 & 112 \\
\hline KRA070 & ALCA- 6 & GRR2 & 16-Dec-07 & 997 & 29.5 & 0.16 & 20.8 & 3.59 & 3.66 & 1.14 & 59.0 & 0.22 & 2.82 & 0.51 & 5496 & 3.56 & 138 & 0.17 & 1.81 & 93 & 0.94 & 0.35 & 13.9 & 43 & 8 \\
\hline KRA080 & ALCA- 6 & GRR2 & 16-Dec-07 & 992 & 29.0 & 0.16 & 17.4 & 3.54 & 3.20 & 1.11 & 58.7 & 0.24 & 2.81 & 0.50 & 5473 & 3.67 & 138 & 0.16 & 1.80 & 93 & 0.95 & 0.37 & 13.9 & 42 & 119 \\
\hline KRA091 & ALCA- 6 & GRR2 & 16-Dec-07 & 989 & 28.3 & 0.16 & 18.7 & 3.54 & 3.62 & 1.08 & 56.9 & 0.23 & 2.78 & 0.48 & 5364 & 3.45 & 137 & 0.17 & 1.76 & 82 & 0.93 & 0.35 & 13.7 & 41 & 107 \\
\hline KRA003 & ALCA-2 & GRR2 & 16-Dec-07 & 984 & 43.8 & 0.27 & 24.1 & 4.27 & 4.00 & 1.25 & 82.5 & 0.41 & 2.77 & 0.66 & 7275 & 4.81 & 145 & 0.13 & 2.01 & 180 & 0.96 & 0.36 & 16.5 & 44 & 167 \\
\hline KRA004 & ALCA-2 & GRR2 & 16-Dec-07 & 974 & 43.8 & 0.17 & 26.5 & 4.24 & 4.03 & 1.31 & 82.3 & 0.45 & 2.80 & 0.64 & 7520 & 4.96 & 145 & 0.13 & 2.02 & 181 & 0.96 & 0.38 & 16.5 & 49 & 174 \\
\hline KRA010 & ALCA-3 & GRR2 & 16-Dec-07 & 1004 & 36.5 & 0.20 & 22.7 & 3.79 & 3.40 & 1.26 & 70.3 & 0.46 & 3.48 & 0.69 & 8091 & 4.74 & 129 & 0.28 & 1.87 & 279 & 0.91 & 0.38 & 13.7 & 47 & 9 \\
\hline KRA011 & ALCA-3 & GRR2 & 16-Dec-07 & 1041 & 36.8 & 0.19 & 23.5 & 3.80 & 3.26 & 1.30 & 68.9 & 0.58 & $\begin{array}{l}3.40 \\
3.41\end{array}$ & 0.69 & 8011 & 4.73 & 128 & 0.27 & 1.85 & 260 & 0.90 & 0.38 & 13.6 & 46 & 166 \\
\hline KRA012 & ALCA-3 & GRR2 & 16-Dec-07 & 1019 & 37.7 & 0.19 & 21.9 & 3.90 & 3.40 & 1.31 & 70.5 & 0.58 & 3.48 & 0.70 & 8152 & 4.66 & 128 & 0.27 & 1.89 & 273 & 0.91 & 0.38 & 13.8 & 47 & 171 \\
\hline KRA013 & ALCA-3 & GRR2 & 16-Dec-07 & 1029 & 37.3 & 0.19 & 23.4 & 3.80 & 3.18 & 1.40 & 69.5 & 0.48 & 3.44 & 0.70 & 8072 & 4.59 & 129 & 0.26 & 1.87 & 265 & 0.90 & 0.39 & 13.8 & 46 & 163 \\
\hline KRA094 & ALCA-3 & GRR2 & 16-Dec-07 & 1037 & 36.5 & 0.21 & 21.9 & 3.86 & 3.46 & 1.31 & 68.8 & 0.56 & 3.36 & 0.68 & 7939 & 4.63 & 126 & 0.25 & 1.84 & 273 & 0.90 & 0.37 & 13.6 & 45 & 153 \\
\hline KRA097 & ALCA-4 & GRR2 & 16-Dec-07 & 1029 & 23.6 & 0.17 & 13.9 & 2.76 & 2.33 & 0.98 & 46.0 & 0.41 & 2.69 & 0.48 & 6077 & 3.06 & 105 & 0.38 & 1.85 & 177 & 0.66 & 0.30 & 7.9 & 37 & \\
\hline KRAO988 & ALCA-4 & GRR2 & 16-Dec-07 & 1033 & 23.9 & 0.21 & 16.6 & 2.80 & 2.16 & 1.00 & 46.7 & 0.35 & 2.71 & 0.49 & 5952 & 3.07 & 108 & 0.40 & 1.84 & 194 & 0.67 & 0.31 & 8.0 & 37 & 101 \\
\hline KRAO99 & ALCA-4 & GRR2 & 16-Dec-07 & 1022 & 23.5 & 0.14 & 15.1 & 2.74 & 2.22 & 0.98 & 45.8 & 0.31 & 2.69 & 0.47 & 5690 & 3.06 & 107 & 0.40 & 1.80 & 174 & 0.67 & 0.30 & 7.9 & 38 & 100 \\
\hline KRA100 & ALCA-4 & GRR2 & 16-Dec-07 & 1014 & 23.8 & 0.14 & 16.0 & 2.81 & 1.76 & 0.98 & 46.4 & 0.42 & 2.71 & 0.48 & 5683 & 3.04 & 107 & 0.39 & 1.82 & 178 & 0.67 & 0.30 & 8.0 & 39 & 101 \\
\hline KRA103 & ALCA-4 & GRR2 & 16-Dec-07 & 1020 & 23.6 & 0.21 & 15.6 & 2.79 & 2.17 & 0.99 & 46.1 & 0.48 & 2.68 & 0.49 & 5788 & 2.97 & 107 & 0.38 & 1.83 & 197 & 0.67 & 0.29 & 8.0 & 35 & 102 \\
\hline KRA104 & ALCA-5 & GRR2 & 16-Dec-07 & 1029 & 34.0 & 0.16 & 22.0 & 3.72 & 3.58 & 1.05 & 67.1 & 0.18 & 2.72 & 0.58 & 5585 & 3.51 & 129 & 0.16 & 1.77 & 149 & 0.92 & 0.35 & 13.9 & 42 & \\
\hline KRA105 & ALCA-5 & GRR2 & 16-Dec-07 & 1017 & 34.6 & 0.15 & 21.3 & 3.77 & 3.55 & 1.10 & 67.7 & 0.18 & 2.76 & 0.60 & 5643 & 3.47 & 130 & 0.15 & 1.79 & 153 & 0.92 & 0.36 & 14.1 & 43 & \\
\hline KRA106 & ALCA-5 & GRR2 & 16-Dec-07 & 973 & 30.3 & 0.15 & 19.6 & 3.58 & 3.62 & 1.04 & 61.0 & 0.15 & 2.80 & 0.55 & 5350 & 3.15 & 131 & 0.15 & 1.75 & 135 & 0.92 & 0.36 & 13.6 & 41 & 2 \\
\hline KRA107 & ALCA-5 & GRR2 & 16-Dec-07 & 1038 & 30.8 & 0.16 & 20.7 & 3.62 & 3.61 & 1.07 & 62.5 & 0.13 & 2.90 & 0.57 & 5413 & 3.33 & 134 & 0.16 & 1.79 & 121 & 0.96 & 0.36 & 13.9 & 42 & 0 \\
\hline KRA108 & ALCA-5 & GRR2 & 16-Dec-07 & 1014 & 30.6 & 0.15 & 21.9 & 3.58 & 3.45 & 1.12 & 61.2 & 0.14 & 2.90 & 0.58 & 5308 & 3.26 & 133 & 0.16 & 1.76 & 132 & 0.94 & 0.34 & 13.8 & 42 & 2 \\
\hline KRA109 & ALCA-5 & GRR2 & 16-Dec-07 & 1012 & 30.9 & 0.15 & 20.4 & 3.66 & 3.77 & 1.08 & 60.7 & 0.13 & 2.83 & 0.56 & 5310 & 3.27 & 132 & 0.16 & 1.76 & 130 & 0.93 & 0.35 & 13.7 & 41 & 11 \\
\hline KRA110 & ALCA-5 & GRR2 & 16-Dec-07 & 1018 & 30.5 & 0.16 & 21.0 & 3.63 & 3.71 & 1.07 & 59.9 & 0.13 & 2.82 & 0.54 & 5202 & 3.33 & 131 & 0.15 & 1.73 & 131 & 0.92 & 0.35 & 13.6 & 41 & 104 \\
\hline KRA111 & ALCA-5 & GRR2 & 16-Dec-07 & 1009 & 30.8 & 0.15 & 20.2 & 3.60 & 3.61 & 1.10 & 61.3 & 0.12 & 2.84 & 0.56 & 5318 & 3.39 & 132 & 0.16 & 1.77 & 134 & 0.95 & 0.36 & 13.8 & 46 & 108 \\
\hline KRA112 & ALCA-5 & GRR2 & 16-Dec-07 & 1013 & 29.8 & 0.15 & 19.8 & 3.57 & 3.59 & 1.06 & 59.3 & 0.12 & 2.79 & 0.54 & 5127 & 3.11 & 129 & 0.16 & 1.70 & 125 & 0.92 & 0.34 & 13.4 & 40 & 120 \\
\hline RA101 & ALCA-7 & GRR2 & 16-Dec-07 & 812 & 30.7 & 0.18 & 19.8 & 3.37 & 4.72 & 1.28 & 57.1 & 0.32 & 4.98 & 0.46 & 4959 & 3.06 & 159 & 0.35 & 1.85 & 150 & 1.17 & 0.35 & 17.0 & 31 & \\
\hline
\end{tabular}

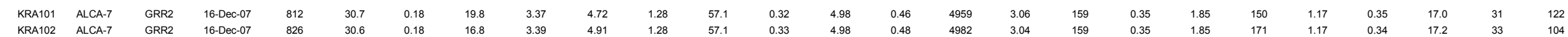




\begin{tabular}{|c|c|c|c|c|c|c|c|c|c|c|c|c|c|c|c|c|c|c|c|c|c|}
\hline ANID & Sub-source & Date & Al2O3 $3(\%)$ & $\mathrm{CaO}(\%)$ & $\mathrm{Fe} 2 \mathrm{O} 3(\%)$ & K2O (\%) & Mgo (\%) & Mno (\%) & Na2O $\%$ ) & P205 (\%) & sior $(\%)$ & Tio2 $(\%)$ & Ва (PPM) & $\operatorname{cr}(\mathrm{PPM})$ & Nb (PPM) & $\mathrm{Rb}(\mathrm{PPM})$ & $\operatorname{sr}($ PPM) & $Y(P P M)$ & $\mathrm{Zn}(\mathrm{PPM})$ & $\operatorname{Zr}(\mathrm{PPM})$ & Sum $(\%)$ \\
\hline $\begin{array}{l}\text { KRA-001 } \\
\text { KA-001 }\end{array}$ & Alca-1 & 12/22/106 10:54 AM & $\begin{array}{l}12.5 \\
125\end{array}$ & 0.61 & 0.89 & 4.4 & 0.11 & 0.063 & 4.3 & 0.026 & 70.2 & 0.129 & 1047 & 12 & 14 & 154 & & 14 & & 104 & 93.40 \\
\hline & & 1/3/07 10:38 PM & 12.5 & 0.61 & 0.84 & 4.4 & 0.10 & 0.062 & 4.3 & 0.028 & 69.7 & 0.128 & 1058 & 3 & 16 & 152 & 81 & 18 & 39 & 110 & 92.78 \\
\hline $\begin{array}{l}\text { KRA-001 maean } \\
\text { KRA-002 }\end{array}$ & & & 12.5 & 0.61 & 0.87 & 4.4 & 0.11 & 0.063 & 4.3 & 0.027 & 70.0 & 0.129 & 1053 & 8 & 15 & ${ }^{153}$ & 81 & 16 & ${ }^{42}$ & 107 & 93.09 \\
\hline $\begin{array}{l}\text { RRA-AUZ } \\
\text { KRA-026 }\end{array}$ & $\begin{array}{l}\text { Allar-1 } \\
\text { Alca-1 }\end{array}$ & $12 / 22106611: 51 \mathrm{AM}$ & $\begin{array}{l}12.7 \\
125\end{array}$ & $\begin{array}{l}0.61 \\
0.60\end{array}$ & $\begin{array}{l}1.00 \\
101\end{array}$ & $\begin{array}{l}4.4 \\
4.4\end{array}$ & $\begin{array}{l}0.122 \\
0.12\end{array}$ & $\begin{array}{l}0.063 \\
0.064\end{array}$ & $\begin{array}{l}4.2 \\
4.4\end{array}$ & $\begin{array}{l}0.027 \\
0.030\end{array}$ & 70.9 & $\begin{array}{l}0.131 \\
0.127\end{array}$ & 1071 & 3 & ${ }^{13}$ & ${ }_{154}^{154}$ & 79 & 16 & 39 & 111 & 94.29 \\
\hline KRA-042 & Alla-1 & $12 / 22 / 0612: 10 \mathrm{PM}$ & $\begin{array}{l}12.5 \\
12.6\end{array}$ & 0.61 & $\begin{array}{l}1.01 \\
1.00\end{array}$ & $\begin{array}{l}\begin{array}{l}4.4 \\
4.4\end{array}\end{array}$ & 0.12 & $\begin{array}{l}0.004 \\
0.064\end{array}$ & $\begin{array}{l}4.4 \\
4.2\end{array}$ & 0.027 & $\begin{array}{l}6590 \\
699\end{array}$ & 0.129 & $\begin{array}{l}1050 \\
1086\end{array}$ & 11 & ${ }_{14}^{4}$ & ${ }_{156}^{103}$ & 列 & 14 & 列 & 昂 & $\begin{array}{l}93.29 \\
93.17\end{array}$ \\
\hline KRA-058 & Alca-1 & 12/22/106 12:29 PM & 12.5 & 0.00 & 0.85 & 4.3 & 0.11 & 0.061 & 4.2 & 0.028 & 69.0 & 0.125 & 1068 & $0_{0}$ & 14 & 159 & 78 & 13 & 41 & ${ }_{108}$ & $\begin{array}{l}93.17 \\
91.85\end{array}$ \\
\hline KRA-061 & Alca-1 & 12/2/2/106 12:48 PM & 12.5 & 0.62 & 0.94 & 4.4 & 0.12 & 0.063 & 4.3 & 0.026 & 69.9 & 0.127 & 1074 & 3 & 13 & ${ }_{152}$ & 80 & 15 & 40 & 104 & $\begin{array}{l}19.85 \\
93.13\end{array}$ \\
\hline KRA-065 & Alca-1 & 1/3/07 3:08 PM & 12.5 & 0.61 & 1.00 & 4.5 & 0.10 & 0.063 & 4.2 & 0.026 & 69.8 & 0.130 & 1072 & 3 & 12 & 155 & 80 & 15 & 40 & 106 & 92.99 \\
\hline KRA-068 & Alca-1 & 1/3/07 12:10 PM & 12.5 & 0.61 & 0.93 & 4.4 & 0.11 & 0.063 & 4.2 & 0.028 & 69.7 & 0.133 & 1105 & 0 & 15 & 154 & 79 & 14 & 39 & 107 & 92.78 \\
\hline KRA-091 & Alca-1 & 1/3/07 1:07 PM & 12.5 & 0.61 & 0.96 & 4.4 & 0.12 & 0.062 & 4.2 & 0.026 & 69.6 & 0.129 & 1068 & 12 & 15 & 161 & 79 & 16 & 38 & 107 & 92.79 \\
\hline KRA-092 & Alca-1 & 1/3/07 1:26 PM & 12.6 & 0.62 & 0.95 & 4.5 & 0.11 & 0.062 & 4.2 & 0.026 & 70.0 & 0.124 & 1068 & 2 & 14 & 154 & 82 & 16 & 35 & 102 & 93.31 \\
\hline KRA-093 & Alca-1 & 1/3/07 1:45 PM & 12.5 & 0.63 & 0.91 & 4.4 & 0.11 & 0.062 & 4.2 & 0.027 & 69.5 & 0.123 & 1053 & 12 & 13 & 155 & 82 & 14 & 44 & 102 & 92.60 \\
\hline KRA-095 & & 1/3/107 8:06 PM & 12.5 & 0.61 & 0.93 & 4.4 & 0.12 & 0.064 & 4.3 & 0.029 & 69.9 & & 1091 & 0 & & 158 & 80 & 16 & 43 & 104 & 93.20 \\
\hline KRA-096 & Alca-1 & 1/3/07 2:04 PM & 12.5 & 0.62 & 0.93 & 4.5 & 0.11 & 0.063 & 4.2 & 0.025 & 69.8 & 0.128 & 1104 & 3 & 16 & 150 & 84 & 15 & 30 & 108 & 93.03 \\
\hline KRA-113 & Alca-1 & 12/22/106 10:35 AM & 12.6 & 0.62 & 0.95 & 4.4 & 0.12 & 0.063 & 4.3 & 0.027 & 70.3 & 0.129 & 1091 & 5 & 14 & 149 & 81 & 18 & 35 & 102 & 93.66 \\
\hline KRA-226 & Alca-1 & 4/20/109 1:17 PM & 13.2 & 0.63 & 0.87 & 4.4 & 0.12 & 0.061 & 4.3 & 0.027 & 76.3 & 0.125 & 1029 & 11 & 11 & 145 & 83 & 10 & 55 & 89 & 100.32 \\
\hline KRA-228 & Alca-1 & 4/18/09 2:44 PM & 13.2 & 0.62 & 0.90 & 4.4 & 0.12 & 0.062 & 4.3 & 0.026 & 76.1 & 0.128 & 1024 & 11 & 12 & 146 & 85 & 12 & ${ }^{34}$ & 86 & 99.95 \\
\hline KRA-230 & Alca-1 & 4/19/09 5:05 PM & 13.2 & 0.62 & 0.91 & 4.5 & 0.12 & 0.060 & 4.3 & 0.029 & 76.3 & 0.130 & 1068 & 9 & 12 & 146 & 84 & 10 & 32 & 90 & 100.23 \\
\hline Mo4-072 & Alca-1 & 1/3/07 12:29 PM & 12.6 & 0.62 & 0.92 & 4.4 & 0.11 & 0.063 & 4.3 & 0.026 & 69.9 & 0.130 & 1071 & 14 & 13 & 157 & 77 & 14 & 43 & 106 & 93.17 \\
\hline M04-074 & Alca-1 & 1/3/07 12:48 PM & 12.5 & 0.62 & 0.87 & 4.4 & 0.11 & 0.061 & 4.3 & 0.023 & 69.9 & 0.127 & 1080 & 9 & 13 & 163 & 76 & 11 & 38 & 106 & 93.00 \\
\hline KRA-003 & Alca-2 & 4/291/08 3:59 PM & 13.8 & 0.88 & 1.22 & 4.3 & 0.19 & 0.058 & 4.5 & 0.033 & 73.6 & 0.193 & 1053 & 5 & 13 & 144 & 157 & 12 & 38 & 149 & 99.01 \\
\hline KRA-004 & Alca-2 & 4/29/08 4:18 PM & 13.7 & 0.87 & 1.20 & 4.3 & 0.16 & 0.061 & 4.5 & 0.034 & 73.6 & 0.190 & 1066 & 4 & 14 & 146 & 154 & 15 & 36 & 145 & 98.73 \\
\hline KRA-151 & Alca-2 & 4/21/09 6:52 PM & 13.7 & 0.90 & 1.18 & 4.3 & 0.18 & 0.056 & 4.5 & 0.036 & 73.9 & 0.185 & 1019 & 13 & 13 & 159 & 160 & 11 & 34 & 144 & 99.12 \\
\hline KRA-010 & Alca-3 & 1/13107 9:22 PM & 13.4 & 1.18 & 1.29 & 4.0 & 0.23 & 0.074 & 4.5 & 0.046 & 68.0 & 0.196 & 1142 & 8 & 12 & 143 & 218 & 16 & 48 & 165 & 93.05 \\
\hline KRA-011 & Alca-3 & 1/3107 9:41 PM & 13.5 & 1.17 & 1.30 & 4.0 & 0.23 & 0.074 & 4.5 & 0.046 & 68.3 & 0.191 & 1134 & 11 & 16 & 146 & 216 & 16 & 43 & 168 & 93.37 \\
\hline KRA-012 & Alca-3 & 12/22106 11:32 AM & 13.4 & 1.16 & 1.29 & 4.0 & 0.24 & 0.073 & 4.4 & 0.045 & 68.0 & 0.193 & 1097 & 10 & 12 & 143 & 213 & 13 & 45 & 166 & 93.05 \\
\hline KRA-154 & Alca-3 & 4/17109 4:26 PM & 14.1 & 1.12 & 1.40 & 4.1 & 0.28 & 0.060 & 4.7 & 0.056 & 73.1 & 0.248 & 1045 & 1 & 12 & 147 & & ${ }_{11}$ & 42 & 185 & 99.37 \\
\hline KRA-155 & Alca-3 & 4/29/08 4:37 PM & 14.1 & 1.17 & 1.33 & 4.1 & 0.22 & 0.073 & 4.4 & 0.047 & 72.9 & 0.191 & 1094 & 13 & 11 & 130 & 224 & 16 & 40 & 145 & 98.61 \\
\hline KRA-158 & Alca-3 & 4/29/08 & 14.1 & 1.19 & 1.35 & 4.1 & 0.22 & 0.0 & 4.5 & 0.040 & 73.3 & 0.190 & 1136 & 5 & 11 & 126 & 224 & 17 & 39 & 144 & 99.25 \\
\hline 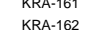 & $\begin{array}{l}\text { Alaca-3 } \\
\text { Alla } 3\end{array}$ & $\begin{array}{l}\text { 4/29980 5:15 PM } \\
\text { 4/29180 5:34 PM }\end{array}$ & $\begin{array}{l}14.1 \\
14.0\end{array}$ & $\begin{array}{l}1.18 \\
1.16\end{array}$ & $\begin{array}{l}1.39 \\
1.34\end{array}$ & $\begin{array}{l}4.0 \\
4.1\end{array}$ & $\begin{array}{l}0.22 \\
0.21\end{array}$ & $\begin{array}{l}0.073 \\
0.073\end{array}$ & $\begin{array}{l}4.4 \\
4.4\end{array}$ & $\begin{array}{l}0.047 \\
0.046\end{array}$ & $\begin{array}{l}73.0 \\
72.5\end{array}$ & $\begin{array}{l}0.191 \\
0.192\end{array}$ & $\begin{array}{l}11113 \\
1100\end{array}$ & $\begin{array}{l}21 \\
16\end{array}$ & $\begin{array}{l}12 \\
12\end{array}$ & 129 & ${ }_{226}^{226}$ & 14 & ${ }^{39}$ & $\begin{array}{l}145 \\
150\end{array}$ & $\begin{array}{c}98.83 \\
98.10\end{array}$ \\
\hline 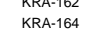 & $\begin{array}{l}\text { Alla-3 } \\
\text { Alla-3 }\end{array}$ & $\begin{array}{l}4 / 2998085: 34 \text { PM } \\
4 / 29 / 08553 \text { PM }\end{array}$ & $\begin{array}{l}14.0 \\
14.1\end{array}$ & $\begin{array}{l}1.16 \\
1.17\end{array}$ & $\begin{array}{l}1.34 \\
1.31\end{array}$ & $\begin{array}{l}4.1 \\
4.1\end{array}$ & $\begin{array}{l}0.21 \\
0.21\end{array}$ & $\begin{array}{l}0.073 \\
0.072\end{array}$ & $\begin{array}{l}4.4 \\
4.4 \\
4.4\end{array}$ & $\begin{array}{l}0.046 \\
0.046\end{array}$ & $\begin{array}{l}72.5 \\
73.0\end{array}$ & $\begin{array}{l}0.192 \\
0.189\end{array}$ & $\begin{array}{l}11113 \\
1113\end{array}$ & $\begin{array}{l}16 \\
15\end{array}$ & $\begin{array}{l}12 \\
12\end{array}$ & $\begin{array}{l}130 \\
128\end{array}$ & $\begin{array}{l}222 \\
225\end{array}$ & ${ }_{13}^{14}$ & ${ }_{37}^{42}$ & 150 & $\begin{array}{l}98.10 \\
98.80\end{array}$ \\
\hline KRA-169 & Alca-3 & 4/29/08 6:12 PM & $\begin{array}{l}14.0 \\
14.0\end{array}$ & 1.17 & $\begin{array}{l}1.31 \\
1.36\end{array}$ & $\begin{array}{l}4.1 \\
4.1\end{array}$ & 0.22 & 0.074 & $\begin{array}{l}4.4 \\
4.4\end{array}$ & 0.044 & 73.1 & o. & 1123 & 10 & ${ }_{11}$ & 128 & 225 & 18 & 41 & 145 & $\begin{array}{l}98.80 \\
98.80\end{array}$ \\
\hline KRA-172 & Alca-3 & 4/29/108 6:31 PM & 14.1 & 1.17 & 1.27 & 4.1 & 0.22 & 0.074 & 4.4 & 0.045 & 72.9 & 0.190 & 1116 & 8 & 10 & 128 & 221 & 15 & 37 & 145 & 98.58 \\
\hline KRA-100 & Alca-4 & 5/1/08 5:48 PM & 13.4 & 0.91 & 0.97 & 3.8 & 0.16 & 0.071 & 4.4 & 0.041 & 74.3 & 0.120 & 1103 & 17 & 8 & 110 & 157 & 12 & 30 & 82 & 98.35 \\
\hline KRA-103 & Alca-4 & 1/3/1/79 9:03 PM & 12.9 & 0.94 & 1.32 & 3.7 & 0.19 & 0.0 & 4.4 & 0.045 & 68.9 & 0.132 & 1145 & 10 & 8 & 120 & 158 & 12 & 37 & 97 & 92.79 \\
\hline KRA-221 & Alca-4 & 5/1108 6:07 PM & 13.5 & 0.92 & 1.00 & 3.8 & 0.16 & 0.072 & 4.4 & 0.040 & 74.6 & 0.127 & 1133 & 4 & 9 & 111 & 160 & 9 & 27 & 85 & 98.84 \\
\hline KRA-242 & Alca-4 & 4/21/09 12:27 PM & 13.6 & 0.91 & 0.92 & 3.8 & 0.18 & 0.0 & 4.5 & 0.038 & 75.9 & 0.123 & 1108 & 3 & 11 & 117 & 160 & 11 & 32 & 78 & 100.12 \\
\hline KRA-252 & Alca-4 & 4/21109 4: & 13.5 & 0.90 & 0.93 & 3.8 & 0. & 0.074 & 4.4. & 0.042 & 75 & 0. & 18 & 9 & 8 & 116 & 155 & 9 & 31 & 81 & 99.54 \\
\hline $\begin{array}{l}\text { KRAA-286 } \\
\text { KRP-097 }\end{array}$ & $\begin{array}{l}\text { Alla-4 } \\
\text { Alca-4 }\end{array}$ & $\begin{array}{l}\text { 1/3/07 8:25 PM } \\
1 / 307844 \mathrm{PM}\end{array}$ & $\begin{array}{l}12.8 \\
129\end{array}$ & $\begin{array}{l}0.90 \\
0.90\end{array}$ & $\begin{array}{l}1.04 \\
1.08\end{array}$ & $\begin{array}{l}3.8 \\
3.8\end{array}$ & $\begin{array}{l}0.18 \\
0.18\end{array}$ & $\begin{array}{l}0.073 \\
0.076\end{array}$ & $\begin{array}{l}4.3 \\
44 \\
4.4\end{array}$ & $\begin{array}{l}0.040 \\
0.040\end{array}$ & 69.4 & $\begin{array}{l}0.130 \\
0.131\end{array}$ & 1105 & $\begin{array}{l}5 \\
6\end{array}$ & 9 & ${ }_{123}^{123}$ & 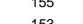 & 16 & 列 & 96 & 92.86 \\
\hline KRA-UGT & Alca-4 4 & 1/3/07 8:44 PM & 12.9 & 0.90 & 1.08 & 3.8 & 0.18 & 0.076 & 4.4 & 0.040 & 69.4 & 0.131 & 1092 & 6 & 9 & 120 & 153 & 12 & ${ }^{32} 2$ & 100 & 93.00 \\
\hline
\end{tabular}


Rademaker Table DR4. WD-XRF concentration data

\begin{tabular}{|c|c|c|c|c|c|c|c|c|c|c|c|c|c|c|c|c|c|c|c|c|c|}
\hline ANID & sub-source & & A1203 (\%) & CaO (\%) & Fe203 $(\%)$ & К2O (\%) & Mgo (\%) & Mno $(\%)$ & $\mathrm{Na2O}(\%)$ & P205 (\%) & $\operatorname{sio}^{2}(\%)$ & Tio2 (\%) & $\mathrm{Ba}(\mathrm{PPM})$ & $\operatorname{cr}(\mathrm{PPM})$ & $\mathrm{Nb}$ (PPM) & $\mathrm{Rb}(\mathrm{PPM})$ & $\operatorname{Sr}(\mathrm{PPM})$ & $Y(\mathrm{PPM})$ & $\mathrm{Zn}(\mathrm{PP} M)$ & $\mathrm{Zr}(\mathrm{PPM})$ & sum (\%) \\
\hline $\begin{array}{l}\text { RAA.190 } \\
\text { KRA-190 }\end{array}$ & $\begin{array}{l}\text { Aca-a } \\
\text { Aca.5 }\end{array}$ & 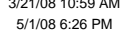 & $\begin{array}{l}13.5 \\
13.3\end{array}$ & $\begin{array}{l}0.15 \\
0.74\end{array}$ & $\begin{array}{l}0.88 \\
0.83\end{array}$ & $\begin{array}{l}4.5 \\
4.4\end{array}$ & $\begin{array}{l}0.09 \\
0.09\end{array}$ & $\begin{array}{l}0.061 \\
0.063\end{array}$ & $\begin{array}{l}4.2 \\
4.1\end{array}$ & $\begin{array}{l}0.025 \\
0.025\end{array}$ & 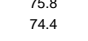 & $\begin{array}{l}0.115 \\
0.113\end{array}$ & $\begin{array}{l}1120 \\
1093\end{array}$ & $\begin{array}{l}19 \\
13\end{array}$ & $\begin{array}{l}11 \\
12\end{array}$ & $\begin{array}{l}131 \\
133\end{array}$ & $\begin{array}{l}120 \\
116\end{array}$ & $\begin{array}{l}12 \\
13\end{array}$ & 年 & $\begin{array}{l}1 \\
77\end{array}$ & $\begin{array}{l}\begin{array}{l}100.03 \\
98.28\end{array} \\
98\end{array}$ \\
\hline $\begin{array}{l}\text { RAA-A09 mean } \\
\text { KRA-106 }\end{array}$ & Alca: 5 & 3/21/108 10:02 AM & $\begin{array}{l}13.4 \\
13.3\end{array}$ & $\begin{array}{l}0.75 \\
0.76\end{array}$ & $\begin{array}{l}0.86 \\
0.87\end{array}$ & $\begin{array}{l}4.5 \\
4.3\end{array}$ & $\begin{array}{l}0.09 \\
0.10\end{array}$ & $\begin{array}{l}0.062 \\
0.061\end{array}$ & $\begin{array}{l}4.2 \\
4.2\end{array}$ & $\begin{array}{l}0.025 \\
0.027\end{array}$ & $\begin{array}{l}75.1 \\
75.2\end{array}$ & $\begin{array}{l}0.114 \\
0.113\end{array}$ & $\begin{array}{l}11107 \\
1087\end{array}$ & $\begin{array}{l}16 \\
7\end{array}$ & $\begin{array}{l}12 \\
10\end{array}$ & $\begin{array}{l}132 \\
131 \\
131\end{array}$ & $\begin{array}{l}118 \\
120\end{array}$ & $\begin{array}{l}13 \\
11\end{array}$ & $\begin{array}{l}32 \\
31\end{array}$ & $\begin{array}{l}74 \\
73\end{array}$ & $\begin{array}{l}99.16 \\
99.10\end{array}$ \\
\hline KRA-107 & $\begin{array}{l}\text { Alca-5 } \\
\text { Acca-5 }\end{array}$ & 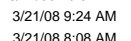 & 13.4 & $\begin{array}{l}0.75 \\
074\end{array}$ & 0.82 & $\begin{array}{l}4.3 \\
45\end{array}$ & 0.10 & 0.062 & 4.3 & 0.027 & $\begin{array}{l}75.3 \\
77.3\end{array}$ & 0.112 & $\begin{array}{l}1103 \\
1125\end{array}$ & 0 & ${ }_{11}^{11}$ & 129 & 114 & 14 & 36 & 77 & 99.34 \\
\hline & & & & & & & & $\begin{array}{l}0.062 \\
0.062\end{array}$ & & & & & $\begin{array}{l}1_{1125} \\
{ }_{1127}\end{array}$ & 14 & 党1 & $\begin{array}{l}131 \\
1220\end{array}$ & $\begin{array}{l}116 \\
1119\end{array}$ & & $\begin{array}{l}35 \\
3{ }_{30}\end{array}$ & & $\begin{array}{l}99.52 \\
9950\end{array}$ \\
\hline KRA-112 & $\begin{array}{l}\text { Acalo-s } \\
\text { Acca-5 }\end{array}$ & 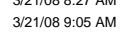 & $\begin{array}{l}13.4 \\
13.5\end{array}$ & 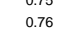 & $\begin{array}{l}0.95 \\
0.95\end{array}$ & 年.3. & $\begin{array}{l}0.10 \\
0.09\end{array}$ & $\begin{array}{l}0.062 \\
0.061\end{array}$ & $\begin{array}{l}4.2 \\
4.2\end{array}$ & $\begin{array}{l}0.027 \\
0.024\end{array}$ & $\begin{array}{l}15.44 \\
75.6\end{array}$ & $\begin{array}{l}\text {. } \\
0.1110\end{array}$ & 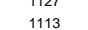 & 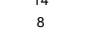 & $\begin{array}{l}n_{10} \\
10\end{array}$ & 126 & $\begin{array}{l}192 \\
1122\end{array}$ & $\begin{array}{l}{ }_{15}^{11} \\
\text {. }\end{array}$ & 海 & $\begin{array}{l}71 \\
73\end{array}$ & .53 \\
\hline KRA-209 & $\begin{array}{l}\text { Alca-5 } \\
\text { Acas. }\end{array}$ & $\begin{array}{l}3212010855.28 \mathrm{PM} \\
3\end{array}$ & 13.5 & 0.79 & 1.00 & 4.3 & 0.11 & 0.062 & 4.3 & 0.031 & 74.6 & 0.122 & 1109 & & 11 & 128 & 124 & 13 & 29 & 87 & .83 \\
\hline & $\begin{array}{l}\text { Acacis } \\
\text { Acta-5.5 }\end{array}$ & 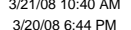 & 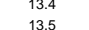 & $\begin{array}{l}0.72 \\
0.75\end{array}$ & $\begin{array}{l}0.84 \\
095\end{array}$ & $\begin{array}{l}4.5 \\
44_{5}\end{array}$ & $\begin{array}{l}0.067 \\
0.14\end{array}$ & $\begin{array}{l}0.063 \\
0.063\end{array}$ & $\begin{array}{l}4.1 \\
44_{3}\end{array}$ & $\begin{array}{l}0.024 \\
0.027\end{array}$ & & $\begin{array}{l}0.1 \\
0.1\end{array}$ & $\begin{array}{l}10466 \\
1097\end{array}$ & & & & & (1) & $\begin{array}{l}34 \\
34\end{array}$ & 7. & 年 \\
\hline KRA-217 & Alca-5 & 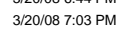 & 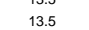 & . & 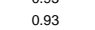 & 4.4 & $\begin{array}{l}0.10 \\
0.09 \\
\end{array}$ & 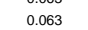 & $\begin{array}{l}4.3 \\
4.3\end{array}$ & - 0.025 & 75 & o.1.15 & 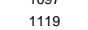 & 13 & 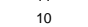 & & ${ }_{11}$ & ${ }_{13}$ & 34 & 79 & 9.14 \\
\hline KRA-219 & $\begin{array}{l}\text { Alca-5 } \\
\text { Acas }\end{array}$ & 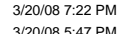 & 13.5 & 0.75 & 0.92 & 4.3 & 0.09 & 0.064 & 4.3 & 年 & 75.9 & 0.11 & 11144 & 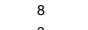 & ${ }_{10}$ & 127 & 11 & 13. & 35 & 74 & $\begin{array}{l}100.18 \\
100.78\end{array}$ \\
\hline $\begin{array}{l}\text { KRA-220 } \\
\text { KRA-225 }\end{array}$ & $\begin{array}{l}\text { Aca-5 } \\
\text { Acca-5 }\end{array}$ & 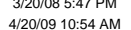 & ${ }_{13}$ & 0.73 & 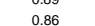 & 4.3 & . & 0.06 & 4.3 & & & 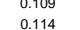 & 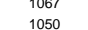 & 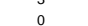 & 12 & & & & 38 & & 1.78 \\
\hline KRA-227 & Alca-5 & 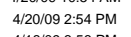 & 13.3 & 0.74 & 0.85 & 4.3 & 0.11 & 0.061 & 4.3 & 0.023 & 75.6 & 0.113 & $\begin{array}{l}1078 \\
1078\end{array}$ & 10 & $\begin{array}{l}12 \\
12\end{array}$ & 140 & 116 & 10 & 34 & 73 & $\begin{array}{l}99.99 \\
99.46\end{array}$ \\
\hline $\begin{array}{l}\text { KAR-2299 } \\
\text { KRA-231 }\end{array}$ & $\begin{array}{l}\text { Alca-5 } \\
\text { Acca-5 }\end{array}$ & 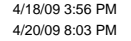 & $\begin{array}{l}\begin{array}{l}13.2 \\
13.5\end{array} \\
1\end{array}$ & $\begin{array}{l}0.73 \\
0.78\end{array}$ & $\begin{array}{l}0.85 \\
0.88\end{array}$ & $\begin{array}{l}4.3 \\
4.3\end{array}$ & $\begin{array}{l}0.11 \\
0.11\end{array}$ & $\begin{array}{l}0.061 \\
0.062\end{array}$ & $\begin{array}{l}4.2 \\
4.4\end{array}$ & $\begin{array}{l}0.025 \\
0.027\end{array}$ & $\begin{array}{l}75.3 \\
75.6 \\
7\end{array}$ & $\begin{array}{l}0.116 \\
0.123 \\
0\end{array}$ & $\begin{array}{l}1009 \\
1075\end{array}$ & 10 & $\begin{array}{l}11 \\
11\end{array}$ & $\begin{array}{l}137 \\
137\end{array}$ & $\begin{array}{l}116 \\
126\end{array}$ & $\begin{array}{c}9 \\
11\end{array}$ & ${ }_{35}^{33}$ & 81 & $\begin{array}{l}11 \\
99 \\
99\end{array}$ \\
\hline KRA-232 & & $4 / 2110910: 21 \mathrm{Am}$ & & & & & & & 4.3 & & 75.4 & 0.106 & & & & 137 & 116 & 10 & 34 & 68 & 32 \\
\hline $\begin{array}{l}\text { KRR-1011 } \\
\text { KRA-102 }\end{array}$ & $\begin{array}{l}\text { Alca-7 } \\
\text { Alcar }\end{array}$ & 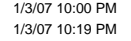 & $\begin{array}{l}12.4 \\
12.4\end{array}$ & $\begin{array}{l}0.900 \\
0.90\end{array}$ & $\begin{array}{l}0.96 \\
0.93\end{array}$ & $\begin{array}{l}\begin{array}{l}4.2 \\
4.4\end{array} \\
4\end{array}$ & $\begin{array}{l}0.12 \\
0.11\end{array}$ & $\begin{array}{l}0.060 \\
0.060\end{array}$ & $\begin{array}{l}3.9 \\
3.8\end{array}$ & $\begin{array}{l}0.021 \\
0.220\end{array}$ & $\begin{array}{l}7.0 \\
6.9 .8 \\
6.8\end{array}$ & $\begin{array}{l}0.102 \\
0.099\end{array}$ & $\begin{array}{l}907 \\
907\end{array}$ & $\begin{array}{c}5 \\
11\end{array}$ & $\begin{array}{l}16 \\
14\end{array}$ & $\begin{array}{l}183 \\
178\end{array}$ & $\begin{array}{l}137 \\
139 \\
139\end{array}$ & $\begin{array}{l}17 \\
17\end{array}$ & $\begin{array}{l}{ }_{31}^{28} \\
31\end{array}$ & $\begin{array}{l}85 \\
82\end{array}$ & $\begin{array}{l}80 \\
61 \\
61\end{array}$ \\
\hline $\begin{array}{l}\text { KRA-1899 } \\
\text { KRAR-195 }\end{array}$ & $\begin{array}{l}\text { Alca-7 } \\
\text { Aclca-7 }\end{array}$ & 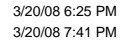 & $\begin{array}{l}13.1 \\
13.1 \\
1.1\end{array}$ & $\begin{array}{l}0.91 \\
0.91\end{array}$ & $\begin{array}{l}0.81 \\
0.80\end{array}$ & $\begin{array}{l}4.3 \\
4.3\end{array}$ & $\begin{array}{l}0.11 \\
0.10\end{array}$ & $\begin{array}{l}0.0 .099 \\
0.059\end{array}$ & $\begin{array}{l}3.9 \\
3.9\end{array}$ & $\begin{array}{l}0.021 \\
0.020\end{array}$ & 75.9 & $\begin{array}{l}0.100 \\
0.101\end{array}$ & $\begin{array}{l}879 \\
877\end{array}$ & & $\begin{array}{l}{ }_{13}^{13} \\
11\end{array}$ & $\begin{array}{l}154 \\
150\end{array}$ & $\begin{array}{l}139 \\
142 \\
142\end{array}$ & $\begin{array}{l}13 \\
13 \\
13\end{array}$ & 24 & 74 & .38 \\
\hline KRA-197 & Acca 7 & 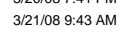 & 13.1 & 然 & 0.78 & 4.3 & 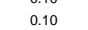 & .0.58 & 3.9. & 然21 & 75.8 & 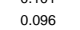 & 896 & 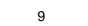 & ${ }_{12}$ & ${ }_{158}$ & 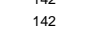 & 13. & ${ }_{20}$ & 75 & .1.16 \\
\hline KRA-198 & Alca-7 & 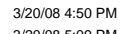 & 13.0 & 0.89 & 0.78 & 4.3 & 0.09 & 0.059 & 3.9 & 0.024 & 75.2 & 0.102 & 869 & 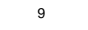 & 15 & 199 & 100 & 15 & 20 & 要 & 98.42 \\
\hline & 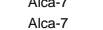 & & & & & 4.3 & 0.09 & & & & & & 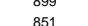 & & & & & & & & 93 \\
\hline KRA-238 & Alca-7 & 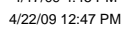 & 12.9 & 0.89 & $\begin{array}{l}0.82 \\
0.80\end{array}$ & 4.3 & 0.11 & $\begin{array}{l}0.0 .099 \\
0.060\end{array}$ & $\begin{array}{l}3.9 \\
3.9\end{array}$ & $\begin{array}{l}0.0219 \\
0.021\end{array}$ & 75.5 & $\begin{array}{l}0.094 \\
0.097\end{array}$ & $\begin{array}{l}891 \\
891\end{array}$ & 9 & $\begin{array}{l}12 \\
12 \\
\end{array}$ & 172 & 143 & 13 & 26 & 72 & .64 \\
\hline ANID & subbsource & & A1203 (\%) & CaO (\%) & Fe203 $(\%)$ & К20 (\%) & Mgo (\%) & Mno (\%) & $\mathrm{N} 20 \mathrm{O}(\%)$ & P205 (\%) & $\operatorname{sio2}(\%)$ & Tio2 (\%) & $\mathrm{Ba}(\mathrm{PPM})$ & $\operatorname{cr}(\mathrm{PPM})$ & $\mathrm{Nb}$ (PPM) & $\mathrm{Rb}(\mathrm{PPM})$ & $\operatorname{Sr}($ PPM) & $Y$ (PPM) & $\mathrm{zn}(\mathrm{PPM})$ & $\operatorname{zr}(\mathrm{PPM})$ & Sum $(\%)$ \\
\hline $\begin{array}{l}\text { Standards } \\
\text { SBG12 }\end{array}$ & & 1212220661:07 PM & 14.9 & 1.14 & 1.48 & 5.8 & 0.29 & 0.063 & 3.7 & 0.172 & 65.7 & 0.186 & 468 & 8 & 28 & 474 & 100 & ${ }_{18}^{18}$ & 40 & 164 & 93.50 \\
\hline 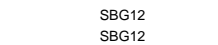 & & 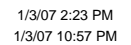 & $\begin{array}{l}14.8 \\
1.48\end{array}$ & $\begin{array}{l}1.15 \\
1.15\end{array}$ & $\begin{array}{l}1.48 \\
1.50 \\
\end{array}$ & $\begin{array}{l}5.8 \\
5.8\end{array}$ & $\begin{array}{l}0.30 \\
0.30\end{array}$ & $\begin{array}{l}0.064 \\
0.064\end{array}$ & $\begin{array}{l}3.7 \\
3.8\end{array}$ & 0.163 & $\begin{array}{l}65.6 \\
65.6\end{array}$ & $\begin{array}{l}0.185 \\
0.190\end{array}$ & 465 & ${ }_{8}^{18}$ & ${ }_{28}$ & 478 & $\begin{array}{l}104 \\
106\end{array}$ & ${ }_{20}^{17}$ & 45 & 168 & 93.42 \\
\hline mean & & & & & & 5.8 & 0.30 & 0.0 & 3.7 & & 65.7 & 0.187 & 456 & 11 & 28 & 476 & 103 & ${ }_{18}^{18}$ & 42 & 167 & 44 \\
\hline $\begin{array}{l}\text { std dev } \\
\text { RSD }\end{array}$ & & & $\begin{array}{l}0.0 \\
0.1\end{array}$ & $\begin{array}{l}0.01 \\
0.5\end{array}$ & $\begin{array}{l}0.01 \\
0.8\end{array}$ & $\begin{array}{l}0.0 \\
0.1\end{array}$ & $\begin{array}{l}0.01 \\
1.9\end{array}$ & $\begin{array}{l}0.001 \\
0.9\end{array}$ & $\begin{array}{l}0.0 \\
0.7\end{array}$ & $\begin{array}{l}0.005 \\
2.8\end{array}$ & $\begin{array}{l}0.1 \\
0.1\end{array}$ & $\begin{array}{l}0.003 \\
1.4\end{array}$ & $\begin{array}{l}19 \\
4.1\end{array}$ & $\begin{array}{c}6 \\
50.9\end{array}$ & $\begin{array}{c}1 \\
2.0\end{array}$ & $\begin{array}{c}2 \\
0.4\end{array}$ & $\begin{array}{c}3 \\
3.0\end{array}$ & $\begin{array}{c}2 \\
8.3\end{array}$ & $\begin{array}{c}3 \\
6.9\end{array}$ & $\begin{array}{c}3 \\
1.8 \\
\end{array}$ & $\begin{array}{l}0.06 \\
0.1\end{array}$ \\
\hline rd mean values & & & 15.4 & 1.14 & 1.43 & 5.6 & 0.29 & 0.06 & 3.8 & 0.171 & 71.1 & 0.184 & 451 & 4 & 24 & 422 & 106 & 17 & 42 & 138 & \\
\hline 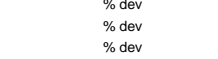 & & 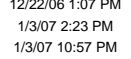 & $\begin{array}{l}3.8 \\
4.0 \\
3.9\end{array}$ & $\begin{array}{l}0.0 \\
0.9 \\
0.9\end{array}$ & $\begin{array}{l}3.5 \\
3.5 \\
4.9\end{array}$ & $\begin{array}{l}2.0 \\
2.0 \\
2.1\end{array}$ & $\begin{array}{l}0.0 \\
3.4 \\
3.4\end{array}$ & $\begin{array}{l}5.0 \\
5.0 \\
6.7\end{array}$ & $\begin{array}{l}1.1 \\
0.8 \\
0.3\end{array}$ & $\begin{array}{l}0.6 \\
3.5 \\
4.7\end{array}$ & $\begin{array}{l}7.6 \\
7.7 \\
7.7\end{array}$ & $\begin{array}{l}1.1 \\
0.5 \\
3.3\end{array}$ & $\begin{array}{l}3.8 \\
3.8 \\
3.1\end{array}$ & $\begin{array}{l}100.0 \\
350.0 \\
1300.0\end{array}$ & $\begin{array}{l}20.8 \\
10.7 \\
1.7\end{array}$ & $\begin{array}{l}12.6 \\
1.3 .3\end{array}$ & $\begin{array}{l}5.7 \\
1.9 \\
0.0\end{array}$ & $\begin{array}{l}5.9 \\
0.0 \\
1.7 .6\end{array}$ & $\begin{array}{l}4.8 \\
4.8 \\
7.1\end{array}$ & $\begin{array}{l}1.8 .8 \\
2.2 .2 \\
2.1 .7\end{array}$ & $\begin{array}{l}\text { N.A. } \\
\text { N.A. } \\
\text { NA. }\end{array}$ \\
\hline$G_{2}$ & & 3/21108 8:46 AM & 15.2 & 1.95 & 2.67 & 4.5 & 0.75 & 0.032 & 4.0 & & 68.7 & 0.490 & 1952 & 14 & 13 & 171 & 493 & 11 & 70 & 320 & \\
\hline $\begin{array}{l}62 \\
62\end{array}$ & & 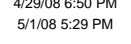 & $\begin{array}{l}15.1 \\
15.1\end{array}$ & $\begin{array}{l}1.94 \\
1.95\end{array}$ & 2.68 & 年.5 & $\begin{array}{l}0.16 \\
0.75\end{array}$ & 作 0.0345 & $\begin{array}{l}4.0 \\
3.9 \\
3.9\end{array}$ & $\begin{array}{l}0.144 \\
0.142 \\
0\end{array}$ & $\begin{array}{l}68.1 \\
68.0\end{array}$ & $\begin{array}{l}0.0466 \\
0.489\end{array}$ & $\begin{array}{l}19903 \\
1993\end{array}$ & $\begin{array}{l}6 \\
15 \\
15\end{array}$ & 年 & $\begin{array}{l}171 \\
171 \\
772\end{array}$ & 491 & $\begin{array}{l}\text { 住 } \\
10\end{array}$ & $\begin{array}{l}88 \\
84\end{array}$ & $\begin{array}{l}329 \\
329\end{array}$ & 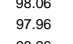 \\
\hline 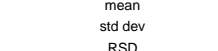 & & & 15.2 & $\begin{array}{l}1.95 \\
0.01\end{array}$ & 2.67 & $\begin{array}{l}4.5 \\
0.0 \\
0.0\end{array}$ & $\begin{array}{l}0.75 \\
0.01\end{array}$ & $\begin{array}{l}0.034 \\
0.002\end{array}$ & $\begin{array}{l}4.0 \\
0.0 \\
0.0\end{array}$ & $\begin{array}{l}0.141 \\
0.003\end{array}$ & $\begin{array}{l}6.3 .3 \\
0.4\end{array}$ & $\begin{array}{l}0.488 \\
0.002\end{array}$ & $\begin{array}{l}{ }_{28}^{162} \\
28\end{array}$ & 12 & 0 & $\begin{array}{c}171 \\
0\end{array}$ & $\begin{array}{c}492 \\
1 \\
4\end{array}$ & 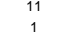 & $\begin{array}{l}81 \\
9 \\
9\end{array}$ & 325 & $\begin{array}{l}{ }^{88.26} \\
0.44\end{array}$ \\
\hline & & & & & & & & & & & & & & & & & & & & 1.4 & .4 \\
\hline arc meal & & & & 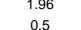 & $\begin{array}{l}2.66 \\
0.64 \\
0.4\end{array}$ & $\begin{array}{l}4.5 \\
07\end{array}$ & .75 & 0.030 & $\begin{array}{l}4.1 \\
22 \\
\end{array}$ & 0.140 & 69.1 & 0.480 & 1880 & $N$ & & 170 & & 11 & & & \\
\hline order & & $\begin{array}{l}412919868.50 \mathrm{PM} \\
5\end{array}$ & 6 & 05 & 0.4 & 0.4 & 1.3 & $\begin{array}{l}13.3 \\
\text {. }\end{array}$ & 2.2 & 2.9 & 1.5 & 1.3 & 3.2 & r. & N.D. & 0.6 & 3.1 & 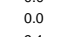 & N.D. & & A. \\
\hline \% dev & & 51/108 5:299 PM & & & & & & & & & & & & & & & & & & & \\
\hline
\end{tabular}




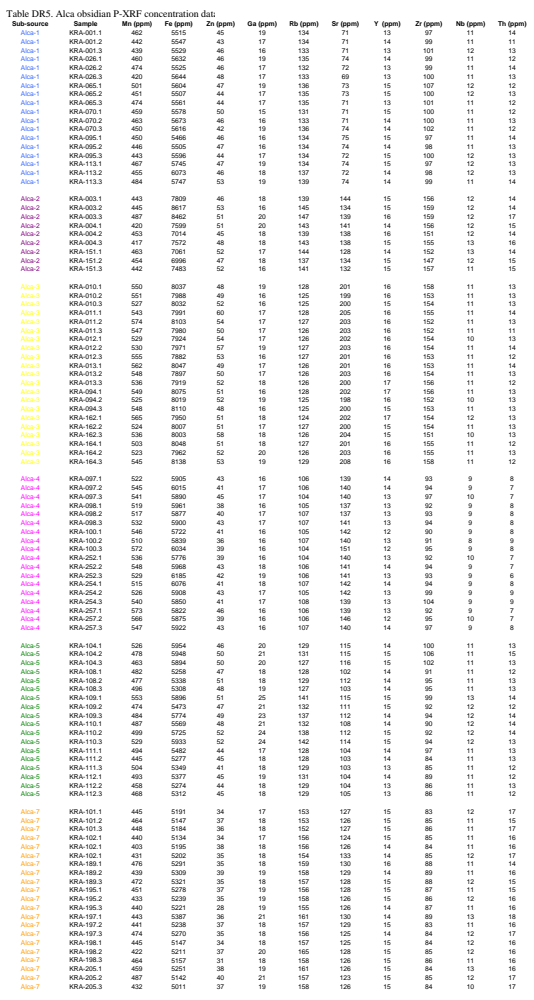




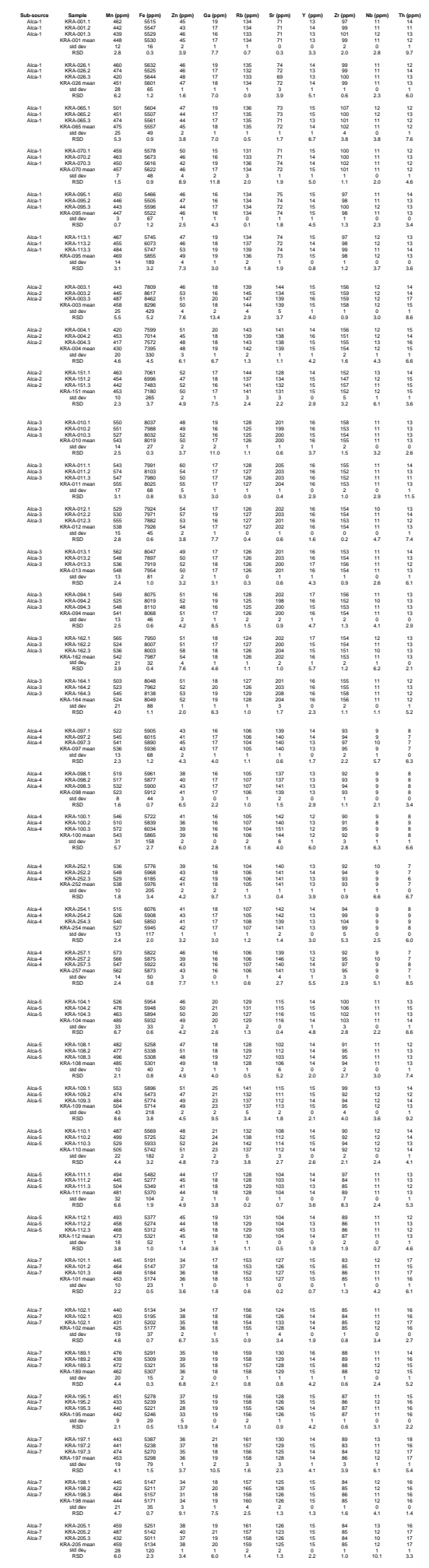




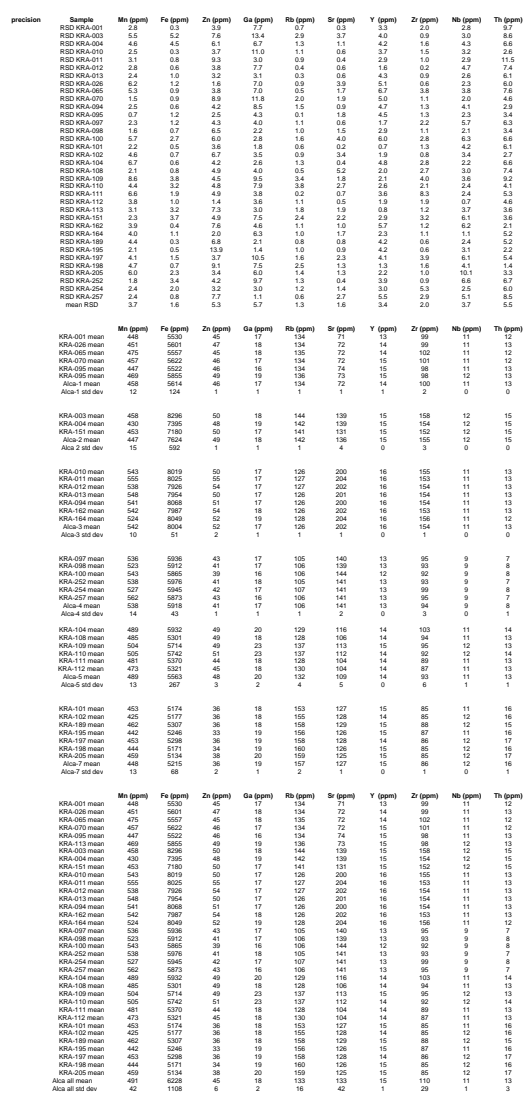


Table DR6. Geographic coordinates for Alca obsidian source samples.

All coordinates are UTM Zone 18S, Datum WGS-84

\begin{tabular}{|c|c|c|c|c|c|c|c|c|}
\hline ANID & Alca sub-source & Northing & Easting & Elev (masl) & ED-XRF & INAA & WD-XRF & P-XRF \\
\hline KRA-001 & Alca-1 & 8325947 & 741049 & 3150 & $x$ & $x$ & $x$ & $x$ \\
\hline KRA-002 & Alca-1 & 8326797 & 740828 & 3050 & $x$ & $x$ & $x$ & \\
\hline KRA-005 & Alca-1 & 8325363 & 747436 & 4448 & $x$ & & & \\
\hline KRA-006 & Alca-1 & 8327982 & 744167 & 4419 & $x$ & & & \\
\hline KRA-007 & Alca-1 & 8326848 & 745229 & 4128 & $\mathrm{X}$ & & & \\
\hline KRA-008 & Alca-1 & 8325570 & 745737 & 4139 & $\mathrm{X}$ & & & \\
\hline KRA-009 & Alca-1 & 8325606 & 747379 & 4292 & $x$ & & & \\
\hline KRA-014 & Alca-1 & 8325622 & 746169 & 4146 & $x$ & & & \\
\hline KRA-015 & Alca-1 & 8326935 & 748391 & 4805 & $x$ & & & \\
\hline KRA-016 & Alca-1 & 8327066 & 748307 & 4826 & $x$ & & & \\
\hline KRA-017 & Alca-1 & 8327309 & 747901 & 4830 & $x$ & & & \\
\hline KRA-018 & Alca-1 & 8327339 & 747738 & 4827 & $x$ & & & \\
\hline KRA-019 & Alca-1 & 8327291 & 747603 & 4818 & $\mathrm{X}$ & & & \\
\hline KRA-020 & Alca-1 & 8327444 & 747676 & 4811 & $x$ & & & \\
\hline KRA-021 & Alca-1 & 8327617 & 747569 & 4808 & $x$ & & & \\
\hline KRA-022 & Alca-1 & 8327617 & 747569 & 4808 & $x$ & & & \\
\hline KRA-023 & Alca-1 & 8327805 & 745923 & 4703 & $x$ & & & \\
\hline KRA-024 & Alca-1 & 8327685 & 745507 & 4673 & $x$ & & & \\
\hline KRA-025 & Alca-1 & 8327330 & 746049 & 4674 & $x$ & & & \\
\hline KRA-026 & Alca-1 & 8327071 & 746168 & 4642 & $x$ & & $x$ & $x$ \\
\hline KRA-027 & Alca-1 & 8326798 & 746241 & 4725 & $x$ & & & \\
\hline KRA-028 & Alca-1 & 8326724 & 746214 & 4749 & $x$ & & & \\
\hline KRA-029 & Alca-1 & 8326724 & 746214 & 4749 & $x$ & & & \\
\hline KRA-030 & Alca-1 & 8326885 & 748248 & 4756 & $x$ & & & \\
\hline KRA-031 & Alca-1 & 8326877 & 748175 & 4770 & $x$ & & & \\
\hline KRA-032 & Alca-1 & 8327005 & 748138 & 4781 & $x$ & & & \\
\hline KRA-033 & Alca-1 & 8327016 & 748085 & 4775 & $x$ & & & \\
\hline KRA-034 & Alca-1 & 8327033 & 748023 & 4769 & $\mathrm{x}$ & & & \\
\hline KRA-035 & Alca-1 & 8327049 & 747914 & 4772 & $x$ & & & \\
\hline KRA-036 & Alca-1 & 8327076 & 747823 & 4766 & $x$ & & & \\
\hline KRA-037 & Alca-1 & 8327090 & 747822 & 4774 & $x$ & & & \\
\hline KRA-038 & Alca-6 (Alca-1) & 8327090 & 747822 & 4774 & $x$ & $\mathrm{x}$ & & \\
\hline KRA-039 & Alca-1 & 8327096 & 747750 & 4772 & $x$ & & & \\
\hline KRA-040 & Alca-1 & 8327086 & 747723 & 4763 & $x$ & & & \\
\hline KRA-041 & Alca-6 (Alca-1) & 8327086 & 747723 & 4763 & $\mathrm{x}$ & $\mathrm{x}$ & & \\
\hline KRA-042 & Alca-1 & 8327086 & 747723 & 4763 & $x$ & & $x$ & \\
\hline KRA-043 & Alca-1 & 8327095 & 747610 & 4757 & $x$ & & & \\
\hline KRA-044 & Alca-1 & 8327097 & 747578 & 4786 & $x$ & & & \\
\hline KRA-045 & Alca-1 & 8327142 & 747514 & 4783 & $x$ & & & \\
\hline KRA-046 & Alca-6 (Alca-1) & 8327198 & 747488 & 4765 & $\mathrm{x}$ & $\mathrm{x}$ & & \\
\hline KRA-047 & Alca-1 & 8327231 & 747479 & 4754 & $x$ & & & \\
\hline KRA-048 & Alca-1 & 8327375 & 747536 & 4773 & $\mathrm{x}$ & & & \\
\hline KRA-049 & Alca-1 & 8327434 & 747616 & 4778 & $x$ & & & \\
\hline KRA-050 & Alca-1 & & & & $\mathrm{x}$ & & & \\
\hline KRA-051 & Alca-1 & 8327115 & 747306 & 4722 & $x$ & & & \\
\hline KRA-052 & Alca-1 & 8327093 & 747214 & 4708 & $\mathrm{x}$ & & & \\
\hline KRA-053 & Alca-1 & 8327038 & 747108 & 4709 & $\mathrm{x}$ & & & \\
\hline KRA-054 & Alca-1 & 8320775 & 746662 & 4726 & $x$ & & & \\
\hline KRA-055 & Alca-1 & 8326707 & 746460 & 4744 & $\mathrm{x}$ & & & \\
\hline KRA-056 & Alca-1 & 8326653 & 746339 & 4761 & $x$ & & & \\
\hline KRA-057 & Alca-1 & 8326608 & 746578 & 4748 & $\mathrm{x}$ & & & \\
\hline KRA-058 & Alca-1 & 8326539 & 746681 & 4783 & $\mathrm{x}$ & & $\mathrm{x}$ & \\
\hline KRA-059 & Alca-1 & 8326539 & 746681 & 4783 & $x$ & & & \\
\hline KRA-060 & Alca-1 & 8326491 & 746696 & 4755 & $\mathrm{x}$ & & & \\
\hline
\end{tabular}


Table DR6. Geographic coordinates for Alca obsidian source samples.

All coordinates are UTM Zone 18S, Datum WGS-84

\begin{tabular}{|c|c|c|c|c|c|c|c|c|}
\hline $\begin{array}{l}\text { KRA-061 } \\
\text { KRA-062 }\end{array}$ & $\begin{array}{c}\text { Alca-1 } \\
\text { Alca-6 (Alca-1) }\end{array}$ & 8326485 & 746729 & 4799 & $x$ & & $x$ & \\
\hline & $\begin{array}{c}\text { Alca-6 (Alca-1) } \\
\text { Alca-1 }\end{array}$ & 8326461 & 746774 & 4820 & $\begin{array}{l}x \\
x\end{array}$ & $x$ & & \\
\hline $\begin{array}{l}\text { KRA-063 } \\
\text { KRA-064 }\end{array}$ & $\begin{array}{l}\text { Alca-1 } \\
\text { Alca-1 }\end{array}$ & $\begin{array}{l}8326461 \\
8326533\end{array}$ & $\begin{array}{l}747018 \\
747199\end{array}$ & $\begin{array}{l}4791 \\
4808\end{array}$ & $\begin{array}{l}x \\
x\end{array}$ & & & \\
\hline KRA-065 & Alca-1 & 8326481 & 747303 & 4817 & $x$ & & $x$ & $x$ \\
\hline KRA-066 & Alca-1 & 8326755 & 747976 & 4791 & $x$ & & & \\
\hline KRA-067 & Alca-1 & 8326781 & 747846 & 4786 & $x$ & & & \\
\hline KRA-068 & Alca-1 & 8326787 & 747759 & 4785 & $x$ & & $\mathrm{x}$ & \\
\hline KRA-069 & Alca-1 & 8326814 & 747653 & 4781 & $x$ & & & \\
\hline KRA-070 & Alca-6 (Alca-1) & 8326756 & 747577 & 4782 & $x$ & $x$ & & $x$ \\
\hline KRA-071 & Alca-1 & 8326483 & 747470 & 4816 & $x$ & & & \\
\hline KRA-072 & Alca-1 & 8326476 & 747585 & 4818 & $\mathrm{x}$ & & & \\
\hline KRA-073 & Alca-1 & 8326664 & 748102 & 4783 & $x$ & & & \\
\hline KRA-074 & Alca-1 & 8326467 & 747321 & 4818 & $x$ & & & \\
\hline KRA-075 & Alca-1 & 8326467 & 747321 & 4818 & $x$ & & & \\
\hline KRA-076 & Alca-1 & 8326604 & 748270 & 4745 & $x$ & & & \\
\hline KRA-077 & Alca-1 & 8326725 & 748358 & 4757 & $x$ & & & \\
\hline KRA-078 & Alca-1 & 8326894 & 748596 & 4759 & $\mathrm{x}$ & & & \\
\hline KRA-079 & Alca-1 & 8326906 & 748626 & 4763 & $x$ & & & \\
\hline KRA-080 & Alca-6 (Alca-1) & 8327016 & 748803 & 4750 & $\mathrm{x}$ & $\mathrm{x}$ & & \\
\hline KRA-081 & Alca-1 & 8327009 & 748884 & 4754 & $x$ & & & \\
\hline KRA-082 & Alca-1 & 8327001 & 748978 & 4737 & $x$ & & & \\
\hline KRA-083 & Alca-1 & 8326813 & 749084 & 4697 & $x$ & & & \\
\hline KRA-084 & Alca-1 & 8326745 & 749096 & 4672 & $x$ & & & \\
\hline KRA-085 & Alca-1 & 8325716 & 747831 & 4394 & $x$ & & & \\
\hline KRA-086 & Alca-1 & 8325655 & 747640 & 4376 & $\mathrm{x}$ & & & \\
\hline KRA-087 & Alca-1 & 8325613 & 747447 & 4355 & $x$ & & & \\
\hline KRA-088 & Alca-1 & 8325420 & 746850 & 4187 & $x$ & & & \\
\hline KRA-089 & Alca-1 & 8325536 & 746278 & 4109 & $x$ & & & \\
\hline KRA-090 & Alca-1 & 8325296 & 745677 & 4009 & $x$ & & & \\
\hline KRA-091 & Alca-6 (Alca-1) & 8325168 & 741040 & 3237 & $\mathrm{x}$ & $x$ & $\mathrm{x}$ & \\
\hline KRA-092 & Alca-1 & 8325033 & 740927 & 3093 & $x$ & & $x$ & \\
\hline KRA-093 & Alca-1 & 8323426 & 740899 & 3318 & $x$ & & $x$ & \\
\hline KRA-095 & Alca-1 & & & & $x$ & $x$ & $x$ & $x$ \\
\hline KRA-096 & Alca-1 & & & & $x$ & & $x$ & \\
\hline KRA-113 & Alca-1 & 8324296 & 740642 & 2889 & $x$ & & $x$ & $x$ \\
\hline KRA-114 & Alca-1 & 8325762 & 739902 & 2720 & $x$ & & & \\
\hline KRA-115 & Alca-1 & 8325762 & 739902 & 2720 & $x$ & & & \\
\hline KRA-116 & Alca-1 & 8325762 & 739902 & 2720 & $x$ & & & \\
\hline KRA-117 & Alca-1 & 8325762 & 739902 & 2720 & $x$ & & & \\
\hline KRA-118 & Alca-1 & 8325762 & 739902 & 2720 & $x$ & & & \\
\hline KRA-119 & Alca-1 & 8325230 & 739956 & 2737 & $x$ & & & \\
\hline KRA-120 & Alca-1 & 8325230 & 739956 & 2737 & $x$ & & & \\
\hline KRA-121 & Alca-1 & 8325230 & 739956 & 2737 & $x$ & & & \\
\hline KRA-122 & Alca-1 & 8325230 & 739956 & 2737 & $x$ & & & \\
\hline KRA-123 & Alca-1 & 8324919 & 739655 & 2773 & $x$ & & & \\
\hline KRA-126 & Alca-1 & 8323949 & 738382 & 2794 & $x$ & & & \\
\hline KRA-127 & Alca-1 & 8324000 & 737743 & 2981 & $x$ & & & \\
\hline KRA-128 & Alca-1 & 8324000 & 737743 & 2981 & $x$ & & & \\
\hline KRA-129 & Alca-1 & 8324000 & 737743 & 2981 & $x$ & & & \\
\hline KRA-130 & Alca-1 & 8324000 & 737743 & 2981 & $x$ & & & \\
\hline KRA-131 & Alca-1 & 8324000 & 737743 & 2981 & $x$ & & & \\
\hline KRA-132 & Alca-1 & 8324000 & 737743 & 2981 & $x$ & & & \\
\hline KRA-133 & Alca-1 & 8324000 & 737743 & 2981 & $x$ & & & \\
\hline
\end{tabular}


Table DR6. Geographic coordinates for Alca obsidian source samples.

All coordinates are UTM Zone 18S, Datum WGS-84

\begin{tabular}{|c|c|c|c|c|c|c|c|c|}
\hline ANID & Alca sub-source & Northing & Easting & Elev (masl) & ED-XRF & INAA & WD-XRF & P-XRF \\
\hline KRA-134 & Alca-1 & 8324000 & 737743 & 2981 & $x$ & & & \\
\hline KRA-135 & Alca-1 & 8324000 & 737743 & 2981 & $x$ & & & \\
\hline KRA-136 & Alca-1 & 8324000 & 737743 & 2981 & $x$ & & & \\
\hline KRA-137 & Alca-1 & 8324000 & 737743 & 2981 & $x$ & & & \\
\hline KRA-139 & Alca-1 & 8323558 & 737878 & 3113 & $x$ & & & \\
\hline KRA-140 & Alca-1 & 8321946 & 737625 & 3853 & $x$ & & & \\
\hline KRA-141 & Alca-1 & 8321946 & 737625 & 3853 & $x$ & & & \\
\hline KRA-142 & Alca-1 & 8321946 & 737625 & 3853 & $x$ & & & \\
\hline KRA-143 & Alca-1 & 8321946 & 737625 & 3853 & $x$ & & & \\
\hline KRA-144 & Alca-1 & 8321946 & 737625 & 3853 & $x$ & & & \\
\hline KRA-145 & Alca-1 & 8325096 & 740087 & 2836 & $x$ & & & \\
\hline KRA-146 & Alca-1 & 8325096 & 740087 & 2836 & $x$ & & & \\
\hline KRA-149 & Alca-1 & 8323444 & 740450 & 3368 & $x$ & & & \\
\hline KRA-150 & Alca-1 & 8323444 & 740450 & 3368 & $x$ & & & \\
\hline KRA-152 & Alca-1 & 8323751 & 738500 & 2867 & $x$ & & & \\
\hline KRA-153 & Alca-1 & 8323751 & 738500 & 2867 & $x$ & & & \\
\hline KRA-175 & Alca-1 & 8298810 & 754916 & 4441 & $x$ & & & \\
\hline KRA-196 & Alca-1 & 8303340 & 754715 & 4462 & $x$ & & & \\
\hline KRA-226 & Alca-1 & 8298823 & 748641 & 4367 & $x$ & & $x$ & \\
\hline KRA-228 & Alca-1 & 8298823 & 748641 & 4367 & $x$ & & $x$ & \\
\hline KRA-230 & Alca-1 & 8300257 & 747957 & 4365 & $x$ & & $x$ & \\
\hline KRA-236 & Alca-1 & 8303276 & 746901 & 4510 & $x$ & & & \\
\hline KRA-237 & Alca-1 & 8303276 & 746901 & 4510 & $x$ & & & \\
\hline KRA-239 & Alca-1 & 8303836 & 749375 & 4454 & $x$ & & & \\
\hline KRA-241 & Alca-1 & 8303836 & 749375 & 4454 & $x$ & & & \\
\hline M04-072 & Alca-1 & 8326510 & 747717 & 4819 & & & $x$ & \\
\hline M04-074 & Alca-1 & 8326644 & 747403 & 4795 & & & $x$ & \\
\hline KRA-003 & Alca-2 & 8323276 & 747030 & 4768 & $x$ & $x$ & $x$ & $x$ \\
\hline KRA-004 & Alca-2 & 8324546 & 746865 & 4422 & $x$ & $\mathrm{x}$ & $x$ & $x$ \\
\hline KRA-151 & Alca-2 & 8323444 & 740450 & 3368 & $x$ & & $x$ & $x$ \\
\hline KRA-010 & Alca-3 & 8327253 & 743697 & 4254 & $x$ & $\mathrm{x}$ & $x$ & $\mathrm{x}$ \\
\hline KRA-011 & Alca-3 & 8327194 & 743602 & 4322 & $x$ & $x$ & $x$ & $x$ \\
\hline KRA-012 & Alca-3 & 8327516 & 743412 & 4302 & $x$ & $x$ & $x$ & $x$ \\
\hline KRA-013 & Alca-3 & 8326353 & 743397 & 4141 & $x$ & $x$ & & $x$ \\
\hline KRA-094 & Alca-3 & 8323426 & 740899 & 3318 & $x$ & $x$ & & $x$ \\
\hline KRA-124 & Alca-3 & 8324946 & 739177 & 2711 & $x$ & & & \\
\hline KRA-125 & Alca-3 & 8324946 & 739177 & 2711 & $x$ & & & \\
\hline KRA-138 & Alca-3 & 8324000 & 737743 & 2981 & $x$ & & & \\
\hline KRA-147 & Alca-3 & 8325096 & 740087 & 2836 & $x$ & & & \\
\hline KRA-148 & Alca-3 & 8325096 & 740087 & 2836 & $x$ & & & \\
\hline KRA-154 & Alca-3 & 8323925 & 738233 & 2848 & $x$ & & $x$ & \\
\hline KRA-155 & Alca-3 & 8327250 & 743695 & 4270 & $x$ & & $x$ & \\
\hline KRA-156 & Alca-3 & 8327250 & 743695 & 4270 & $x$ & & & \\
\hline KRA-157 & Alca-3 & 8327250 & 743695 & 4270 & $x$ & & & \\
\hline KRA-158 & Alca-3 & 8327258 & 743675 & 4289 & $x$ & & $x$ & \\
\hline KRA-159 & Alca-3 & 8327258 & 743675 & 4289 & $x$ & & & \\
\hline KRA-160 & Alca-3 & 8327258 & 743675 & 4289 & $x$ & & & \\
\hline KRA-161 & Alca-3 & 8327281 & 743643 & 4300 & $x$ & & $x$ & \\
\hline KRA-162 & Alca-3 & 8327203 & 743587 & 4330 & $x$ & & $x$ & $x$ \\
\hline KRA-163 & Alca-3 & 8327203 & 743587 & 4330 & $x$ & & & \\
\hline KRA-164 & Alca-3 & 8327517 & 743487 & 4339 & $x$ & & $x$ & $x$ \\
\hline
\end{tabular}


Table DR6. Geographic coordinates for Alca obsidian source samples.

All coordinates are UTM Zone 18S, Datum WGS-84

\begin{tabular}{|c|c|c|c|c|c|c|c|c|}
\hline ANID & Alca sub-source & Northing & Easting & Elev (masl) & ED-XRF & INAA & WD-XRF & P-XRF \\
\hline KRA-165 & Alca-3 & 8327517 & 743487 & 4339 & $x$ & & & \\
\hline KRA-166 & Alca-3 & 8327690 & 743223 & 4330 & $x$ & & & \\
\hline KRA-167 & Alca-3 & 8327690 & 743223 & 4330 & $x$ & & & \\
\hline KRA-168 & Alca-3 & 8327690 & 743223 & 4330 & $x$ & & & \\
\hline KRA-169 & Alca-3 & 8327690 & 743223 & 4330 & $x$ & & $x$ & \\
\hline KRA-170 & Alca-3 & 8327690 & 743223 & 4330 & $x$ & & & \\
\hline KRA-171 & Alca-3 & 8328085 & 743190 & 4337 & $x$ & & & \\
\hline KRA-172 & Alca-3 & 8328085 & 743190 & 4337 & $x$ & & $x$ & \\
\hline KRA-173 & Alca-3 & 8328085 & 743190 & 4337 & $x$ & & & \\
\hline KRA-097 & Alca-4 & 8286722 & 737031 & 4233 & $x$ & $x$ & $x$ & $x$ \\
\hline KRA-098 & Alca-4 & 8286722 & 737031 & 4233 & $x$ & $x$ & & $x$ \\
\hline KRA-099 & Alca-4 & 8286722 & 737031 & 4233 & $x$ & $x$ & & \\
\hline KRA-100 & Alca-4 & 8286722 & 737031 & 4233 & $x$ & $x$ & $x$ & $x$ \\
\hline KRA-103 & Alca-4 & 8286987 & 737432 & 4395 & $x$ & $x$ & $x$ & \\
\hline KRA-221 & Alca-4 & 8286722 & 737031 & 4233 & $x$ & & $x$ & \\
\hline KRA-242 & Alca-4 & & & & $x$ & & $x$ & \\
\hline KRA-252 & Alca-4 & 8287708 & 739198 & 4342 & & & $x$ & $x$ \\
\hline KRA-253 & Alca-4 & 8287708 & 739198 & 4342 & $x$ & & & \\
\hline KRA-254 & Alca-4 & 8287708 & 739198 & 4342 & $x$ & & & $x$ \\
\hline KRA-255 & Alca-4 & 8284949 & 738076 & 4229 & $x$ & & & \\
\hline KRA-256 & Alca-4 & 8286139 & 736690 & 4138 & $x$ & & & \\
\hline KRA-257 & Alca-4 & 8286942 & 737381 & 4381 & $x$ & & & $x$ \\
\hline KRA-258 & Alca-4 & 8268056 & 737073 & 4152 & $x$ & & & \\
\hline KRA-286 & Alca-4 & & & & & & $x$ & \\
\hline KRA-104 & Alca-5 & 8300667 & 755833 & 4519 & $x$ & $x$ & & $x$ \\
\hline KRA-105 & Alca-5 & 8296562 & 747304 & 4332 & $x$ & $x$ & & \\
\hline KRA-106 & Alca-5 & 8293168 & 739142 & 4082 & $x$ & $x$ & $x$ & \\
\hline KRA-107 & Alca-5 & 8292697 & 738733 & 4138 & $x$ & $x$ & $x$ & $x$ \\
\hline KRA-108 & Alca-5 & 8294521 & 742651 & 4286 & $x$ & $x$ & & $x$ \\
\hline KRA-109 & Alca-5 & 8294521 & 742651 & 4286 & $x$ & $x$ & $x$ & $x$ \\
\hline KRA-110 & Alca-5 & 8294521 & 742651 & 4286 & $x$ & $x$ & & $x$ \\
\hline KRA-111 & Alca-5 & 8294514 & 742404 & 4276 & $x$ & $x$ & $x$ & $x$ \\
\hline KRA-112 & Alca-5 & 8294265 & 742539 & 4295 & $x$ & $x$ & $x$ & $x$ \\
\hline KRA-174 & Alca-5 & 8298483 & 754589 & 4435 & $x$ & & & \\
\hline KRA-176 & Alca-5 & 8300246 & 755124 & 4453 & $x$ & & & \\
\hline KRA-190 & Alca-5 & 8303670 & 755690 & 4511 & $x$ & & $x$ & \\
\hline KRA-209 & Alca-5 & 8299145 & 751486 & 4372 & $x$ & & $x$ & \\
\hline KRA-210 & Alca-5 & 8299145 & 751486 & 4372 & $x$ & & & \\
\hline KRA-211 & Alca-5 & 8297773 & 748682 & 4332 & $x$ & & $x$ & \\
\hline KRA-212 & Alca-5 & 8297361 & 748424 & 4345 & $x$ & & $x$ & \\
\hline KRA-213 & Alca-5 & 8294505 & 742852 & 4283 & $x$ & & & \\
\hline KRA-214 & Alca-5 & 8294505 & 742852 & 4283 & $x$ & & & \\
\hline KRA-215 & Alca-5 & 8294505 & 742852 & 4283 & $x$ & & & \\
\hline KRA-217 & Alca-5 & 8294505 & 742852 & 4283 & $x$ & & $x$ & \\
\hline KRA-218 & Alca-5 & 8294505 & 742852 & 4283 & $x$ & & & \\
\hline KRA-219 & Alca-5 & 8294387 & 742375 & 4283 & $x$ & & $x$ & \\
\hline KRA-220 & Alca-5 & 8294530 & 741794 & 4266 & $x$ & & $x$ & \\
\hline KRA-222 & Alca-5 & 8297876 & 748327 & 4404 & $x$ & & & \\
\hline KRA-223 & Alca-5 & 8297876 & 748327 & 4404 & $x$ & & & \\
\hline KRA-224 & Alca-5 & 8297935 & 748327 & 4408 & $x$ & & & \\
\hline KRA-225 & Alca-5 & 8298823 & 748641 & 4367 & $x$ & & $x$ & \\
\hline KRA-227 & Alca-5 & 8298823 & 748641 & 4367 & $x$ & & $x$ & \\
\hline
\end{tabular}


Table DR6. Geographic coordinates for Alca obsidian source samples.

All coordinates are UTM Zone 18S, Datum WGS-84

\begin{tabular}{|c|c|c|c|c|c|c|c|c|}
\hline ANID & Alca sub-source & Northing & Easting & Elev (masl) & ED-XRF & INAA & WD-XRF & P-XRF \\
\hline KRA-229 & Alca-5 & 8300257 & 747957 & 4365 & $x$ & & $x$ & \\
\hline KRA-231 & Alca-5 & 8300257 & 747957 & 4365 & $x$ & & $x$ & \\
\hline KRA-232 & Alca-5 & 8300257 & 747957 & 4365 & $x$ & & $x$ & \\
\hline KRA-233 & Alca-5 & 8298214 & 750038 & 4346 & $x$ & & & \\
\hline KRA-234 & Alca-5 & 8298214 & 750038 & 4346 & $x$ & & & \\
\hline KRA-235 & Alca-5 & 8303276 & 746901 & 4510 & $x$ & & & \\
\hline KRA-240 & Alca-5 & 8303836 & 749375 & 4454 & $x$ & & & \\
\hline KRA-244 & Alca-5 & 8300714 & 751333 & 4375 & $x$ & & & \\
\hline KRA-246 & Alca-5 & 8290483 & 742087 & 4398 & $x$ & & & \\
\hline KRA-247 & Alca-5 & 8290483 & 742087 & 4398 & $x$ & & & \\
\hline KRA-248 & Alca-5 & 8290483 & 742087 & 4398 & $x$ & & & \\
\hline KRA-249 & Alca-5 & 8290483 & 742087 & 4398 & $x$ & & & \\
\hline KRA-250 & Alca-5 & 8290483 & 742087 & 4398 & $x$ & & & \\
\hline KRA-251 & Alca-5 & 8290018 & 741723 & 4464 & $x$ & & & \\
\hline KRA-101 & Alca-7 & 8304076 & 757739 & 4594 & $x$ & $x$ & $x$ & $x$ \\
\hline KRA-102 & Alca-7 & 8304076 & 757739 & 4594 & $x$ & $x$ & $x$ & $x$ \\
\hline KRA-177 & Alca-7 & 8300246 & 755124 & 4453 & $x$ & & & \\
\hline KRA-178 & Alca-7 & 8300246 & 755124 & 4453 & $x$ & & & \\
\hline KRA-179 & Alca-7 & 8302013 & 755328 & 4512 & $x$ & & & \\
\hline KRA-180 & Alca-7 & 8302013 & 755328 & 4512 & $x$ & & & \\
\hline KRA-181 & Alca-7 & 8302517 & 755735 & 4531 & $x$ & & & \\
\hline KRA-182 & Alca-7 & 8302517 & 755735 & 4531 & $x$ & & & \\
\hline KRA-183 & Alca-7 & 8302640 & 756235 & 4536 & $x$ & & & \\
\hline KRA-184 & Alca-7 & 8302640 & 756235 & 4536 & $x$ & & & \\
\hline KRA-185 & Alca-7 & 8302640 & 756235 & 4536 & $x$ & & & \\
\hline KRA-186 & Alca-7 & 8303416 & 756172 & 4585 & $x$ & & & \\
\hline KRA-187 & Alca-7 & 8303416 & 756172 & 4585 & $x$ & & & \\
\hline KRA-188 & Alca-7 & 8303416 & 756172 & 4585 & $x$ & & & \\
\hline KRA-189 & Alca-7 & 8303670 & 755690 & 4511 & $x$ & & $x$ & $x$ \\
\hline KRA-191 & Alca-7 & 8303670 & 755690 & 4511 & $x$ & & & \\
\hline KRA-192 & Alca-7 & 8303670 & 755690 & 4511 & $x$ & & & \\
\hline KRA-193 & Alca-7 & 8303670 & 755690 & 4511 & $x$ & & & \\
\hline KRA-195 & Alca-7 & 8303340 & 754715 & 4462 & $x$ & & $x$ & $x$ \\
\hline KRA-197 & Alca-7 & 8303340 & 754715 & 4462 & $x$ & & $x$ & $x$ \\
\hline KRA-198 & Alca-7 & 8303340 & 754715 & 4462 & $x$ & & $x$ & $x$ \\
\hline KRA-199 & Alca-7 & 8300740 & 751770 & 4394 & $x$ & & & \\
\hline KRA-200 & Alca-7 & 8300740 & 751770 & 4394 & $x$ & & & \\
\hline KRA-201 & Alca-7 & 8300740 & 751770 & 4394 & $x$ & & & \\
\hline KRA-202 & Alca-7 & 8300740 & 751770 & 4394 & $x$ & & & \\
\hline KRA-203 & Alca-7 & 8300740 & 751770 & 4394 & $x$ & & & \\
\hline KRA-204 & Alca-7 & 8300740 & 751770 & 4394 & $x$ & & & \\
\hline KRA-205 & Alca-7 & 8300740 & 751770 & 4394 & $x$ & & $x$ & $x$ \\
\hline KRA-207 & Alca-7 & 8300740 & 751770 & 4394 & $x$ & & & \\
\hline KRA-208 & Alca-7 & 8300740 & 751770 & 4394 & & & $x$ & \\
\hline KRA-238 & Alca-7 & 8303889 & 748521 & 4473 & $x$ & & $x$ & \\
\hline KRA-243 & Alca-7 & 8303494 & 753496 & 4457 & $x$ & & & \\
\hline
\end{tabular}


MAHALANOBIS DISTANCE CALCULATION AND POSTERIOR CLASSIFICATION FOR TWO OR MORE GROUPS.

Date: $10 / 21 / 12$

File: Maha8.txt

Groups are:
1 EDALCA1
2 EDALCA3
3 EDALCA4
$4 \quad$ EDALCA5
5 EDALCA7

Variables used:

$$
\mathrm{Rb} \quad \mathrm{Sr}
$$

Probabilities are jackknifed for specimens included in each group.

The following specimens are in the file EDALCA1

\begin{tabular}{|c|c|c|c|c|c|c|}
\hline \multirow{2}{*}{$\begin{array}{l}\text { ID. NO. } \\
\text { Into: }\end{array}$} & \multicolumn{2}{|c|}{ Probabilities: } & \multirow[b]{2}{*}{ EDALCA4 } & \multirow[b]{2}{*}{ EDALCA5 } & \multirow[b]{2}{*}{ EDALCA7 } & \multirow[b]{2}{*}{ From: } \\
\hline & EDALCA1 & EDALCA3 & & & & \\
\hline $\begin{array}{l}\text { KRA००1 } \\
1\end{array}$ & 78.860 & $\odot .0 \odot \odot$ & $\odot . \odot \odot \odot$ & 0.013 & $\odot . \odot \odot \odot$ & 1 \\
\hline $\begin{array}{l}\text { KRA००2 } \\
1\end{array}$ & 43.042 & $\odot .0 \odot \odot$ & $\odot . \odot \odot \odot$ & $\odot . \odot \odot \odot$ & $\odot . \odot \odot \odot$ & 1 \\
\hline $\begin{array}{l}\text { KRA๑०5 } \\
1\end{array}$ & 97.687 & $0.00 \odot$ & $\odot .0 \odot \odot$ & 0.006 & $\odot .00 \odot$ & 1 \\
\hline $\begin{array}{l}\text { KRA००6 } \\
1\end{array}$ & 12.835 & 0.000 & $\odot .0 \odot \odot$ & 0.000 & 0.000 & 1 \\
\hline $\begin{array}{l}\text { KRA๑०7 } \\
1\end{array}$ & 48.963 & 0.000 & $\odot .0 \odot \odot$ & 0.001 & 0.000 & 1 \\
\hline $\begin{array}{l}\text { KRA๑०8 } \\
1\end{array}$ & 82.279 & $0.00 \odot$ & $\odot .00 \odot$ & 0.002 & 0.000 & 1 \\
\hline $\begin{array}{l}\text { KRA๑०9 } \\
1\end{array}$ & 71.612 & 0.000 & $\odot .0 \odot \odot$ & $\odot .0 \odot 1$ & $\odot . \odot \odot \odot$ & 1 \\
\hline $\begin{array}{l}\text { KRA014 } \\
1\end{array}$ & 74.614 & 0.000 & $\odot .0 \odot \odot$ & 0.002 & 0.000 & 1 \\
\hline $\begin{array}{l}\text { KRA015 } \\
1\end{array}$ & 40.767 & 0.000 & $\odot .0 \odot \odot$ & 0.000 & 0.000 & 1 \\
\hline $\begin{array}{l}\text { KRA๑16 } \\
1\end{array}$ & 59.025 & 0.000 & $\odot .0 \odot \odot$ & 0.001 & 0.000 & 1 \\
\hline $\begin{array}{l}\text { KRA017 } \\
1\end{array}$ & 67.519 & 0.000 & $\odot .0 \odot \odot$ & 0.001 & $\odot . \odot \odot \odot$ & 1 \\
\hline $\begin{array}{l}\text { KRA@18 } \\
1\end{array}$ & 7.304 & 0.000 & $\odot .0 \odot \odot$ & 0.000 & $\odot .00 \odot$ & 1 \\
\hline $\begin{array}{l}\text { KRA019 } \\
1\end{array}$ & 43.792 & 0.000 & $\odot .0 \odot \odot$ & 0.008 & 0.000 & 1 \\
\hline $\begin{array}{l}\text { KRAO20 } \\
1\end{array}$ & 78.304 & 0.000 & $\odot .0 \odot \odot$ & $\odot .0 \odot 5$ & $\odot . \odot \odot \odot$ & 1 \\
\hline KRA०21 & 11.832 & $\odot .0 \odot \odot$ & $\odot .0 \odot \odot$ & 0.000 & 0.000 & 1 \\
\hline
\end{tabular}

1 


\begin{tabular}{|c|c|c|c|c|c|}
\hline $\begin{array}{l}\text { KRAO22 } \\
1\end{array}$ & 9.642 & $\odot . \odot \odot \odot$ & $\odot . \odot \odot \odot$ & $\odot . \odot \odot \odot$ & $\odot .0 \odot \odot$ \\
\hline $\begin{array}{l}\text { KRAO23 } \\
1\end{array}$ & 55.186 & $\odot .00 \odot$ & 0.000 & $\odot .001$ & 0.000 \\
\hline $\begin{array}{l}\text { KRAO24 } \\
1\end{array}$ & 81.532 & $\odot . \odot \odot \odot$ & $\odot . \odot \odot \odot$ & $\odot .0 \odot 1$ & $\odot .0 \odot \odot$ \\
\hline $\begin{array}{l}\text { KRAO25 } \\
1\end{array}$ & 19.030 & 0.000 & 0.000 & 0.078 & $\odot .0 \odot \odot$ \\
\hline $\begin{array}{l}\text { KRAO26 } \\
1\end{array}$ & 29.312 & 0.000 & 0.000 & $\odot .000$ & 0.000 \\
\hline $\begin{array}{l}\text { KRAO27 } \\
1\end{array}$ & 99.098 & $\odot .00 \odot$ & $\odot . \odot \odot \odot$ & $\odot .0 \odot 5$ & $\odot . \odot \odot \odot$ \\
\hline $\begin{array}{l}\text { KRAO28 } \\
1\end{array}$ & 64.326 & 0.000 & 0.000 & 0.001 & $\odot .00 \odot$ \\
\hline $\begin{array}{l}\text { KRA029 } \\
1\end{array}$ & 96.052 & 0.000 & 0.000 & $\odot .002$ & $\odot .00 \odot$ \\
\hline $\begin{array}{l}\text { KRA०30 } \\
1\end{array}$ & 89.935 & $\odot .00 \odot$ & $\odot .00 \odot$ & 0.002 & $\odot .00 \odot$ \\
\hline $\begin{array}{l}\text { KRA031 } \\
1\end{array}$ & 31.775 & $\odot . \odot \odot \odot$ & $\odot . \odot \odot \odot$ & $\odot .095$ & $\odot .0 \odot \odot$ \\
\hline $\begin{array}{l}\text { KRAO32 } \\
1\end{array}$ & 42.583 & $\odot . \odot \odot \odot$ & $\odot . \odot \odot \odot$ & $\odot .00 \odot$ & $\odot .00 \odot$ \\
\hline $\begin{array}{l}\text { KRA०33 } \\
1\end{array}$ & 46.425 & $\odot . \odot \odot \odot$ & $\odot . \odot \odot \odot$ & $\odot . \odot \odot \odot$ & $\odot .00 \odot$ \\
\hline $\begin{array}{l}\text { KRA@34 } \\
1\end{array}$ & 72.211 & $\odot .0 \odot \odot$ & $\odot .00 \odot$ & $\odot .0 \odot 5$ & $0.00 \odot$ \\
\hline $\begin{array}{l}\text { KRAO35 } \\
1\end{array}$ & 41.917 & $\odot . \odot \odot \odot$ & $\odot . \odot \odot \odot$ & $\odot .062$ & $\odot . \odot \odot \odot$ \\
\hline $\begin{array}{l}\text { KRA०36 } \\
1\end{array}$ & 39.769 & $\odot . \odot \odot \odot$ & $\odot . \odot \odot \odot$ & ๑. . 017 & $\odot . \odot \odot \odot$ \\
\hline $\begin{array}{l}\text { KRA०37 } \\
1\end{array}$ & 45.569 & $\odot .0 \odot \odot$ & $\odot .00 \odot$ & $\odot .0 \odot 3$ & $\odot . \odot \odot \odot$ \\
\hline $\begin{array}{l}\text { KRA@38 } \\
1\end{array}$ & 22.893 & $\odot . \odot \odot \odot$ & $\odot . \odot \odot \odot$ & $\odot .0 \odot 3$ & 0.000 \\
\hline $\begin{array}{l}\text { KRA@39 } \\
1\end{array}$ & 35.426 & $\odot . \odot \odot \odot$ & $\odot .0 \odot \odot$ & $\odot . \odot \odot \odot$ & $\odot . \odot \odot \odot$ \\
\hline $\begin{array}{l}\text { KRA०40 } \\
1\end{array}$ & 53.436 & $\odot . \odot \odot \odot$ & $\odot . \odot \odot \odot$ & $\odot .027$ & $\odot .0 \odot \odot$ \\
\hline $\begin{array}{l}\text { KRA०41 } \\
1\end{array}$ & 1.017 & $\odot . \odot \odot \odot$ & $\odot . \odot \odot \odot$ & 0.000 & $0.00 \odot$ \\
\hline $\begin{array}{l}\text { KRA०42 } \\
1\end{array}$ & 58.164 & $\odot . \odot \odot \odot$ & $\odot . \odot \odot \odot$ & $\odot . \odot \odot \odot$ & 0.000 \\
\hline $\begin{array}{l}\text { KRA०43 } \\
1\end{array}$ & 78.242 & $\odot . \odot \odot \odot$ & $\odot . \odot \odot \odot$ & $\odot .0 \odot 3$ & $\odot . \odot \odot \odot$ \\
\hline $\begin{array}{l}\text { KRA०44 } \\
1\end{array}$ & 43.025 & $\odot . \odot \odot \odot$ & $\odot .00 \odot$ & $\odot . \odot \odot \odot$ & $\odot . \odot \odot \odot$ \\
\hline $\begin{array}{l}\text { KRA०45 } \\
1\end{array}$ & 75.725 & $\odot . \odot \odot \odot$ & $\odot .00 \odot$ & $\odot .0 \odot 1$ & $\odot . \odot \odot \odot$ \\
\hline $\begin{array}{l}\text { KRA०46 } \\
1\end{array}$ & $\odot .289$ & $\odot . \odot \odot \odot$ & $\odot .001$ & $\odot .0 \odot 5$ & $\odot . \odot \odot \odot$ \\
\hline $\begin{array}{l}\text { KRA@47 } \\
1\end{array}$ & 63.824 & $\odot . \odot \odot \odot$ & $\odot . \odot \odot \odot$ & 0.006 & $\odot .0 \odot \odot$ \\
\hline $\begin{array}{l}\text { KRA०48 } \\
1\end{array}$ & $\odot .992$ & $\odot . \odot \odot \odot$ & $\odot . \odot \odot \odot$ & $\odot . \odot \odot \odot$ & $\odot . \odot \odot \odot$ \\
\hline
\end{tabular}




\begin{tabular}{|c|c|c|c|c|c|}
\hline $\begin{array}{l}\text { KRA๑49 } \\
1\end{array}$ & 48.469 & $\odot . \odot \odot \odot$ & $\odot . \odot \odot \odot$ & 0.000 & $\odot . \odot \odot \odot$ \\
\hline $\begin{array}{l}\text { KRA050 } \\
1\end{array}$ & 21.213 & $\odot .0 \odot \odot$ & $\odot . \odot \odot \odot$ & 0.000 & $\odot .00 \odot$ \\
\hline $\begin{array}{l}\text { KRA051 } \\
1\end{array}$ & 64.305 & $\odot . \odot \odot \odot$ & $\odot . \odot \odot \odot$ & 0.001 & $\odot . \odot \odot \odot$ \\
\hline $\begin{array}{l}\text { KRA052 } \\
1\end{array}$ & 87.335 & $\odot .00 \odot$ & 0.000 & 0.002 & 0.000 \\
\hline $\begin{array}{l}\text { KRA053 } \\
1\end{array}$ & 48.183 & $\odot .000$ & 0.000 & 0.011 & $\odot .000$ \\
\hline $\begin{array}{l}\text { KRA०54 } \\
1\end{array}$ & 75.506 & $\odot .00 \odot$ & $\odot .000$ & 0.001 & $\odot .000$ \\
\hline $\begin{array}{l}\text { KRA055 } \\
1\end{array}$ & 87.407 & $\odot .000$ & 0.000 & 0.001 & 0.000 \\
\hline $\begin{array}{l}\text { KRA๑56 } \\
1\end{array}$ & 10.864 & $\odot .000$ & $\odot .0 \odot \odot$ & 0.000 & 0.000 \\
\hline $\begin{array}{l}\text { KRA०57 } \\
1\end{array}$ & 18.199 & $\odot .000$ & $\odot .0 \odot \odot$ & 0.000 & $\odot .000$ \\
\hline $\begin{array}{l}\text { KRA058 } \\
1\end{array}$ & 48.280 & $\odot .00 \odot$ & $\odot .000$ & 0.000 & 0.000 \\
\hline $\begin{array}{l}\text { KRA๑59 } \\
1\end{array}$ & 30.130 & $\odot .000$ & $\odot .000$ & 0.000 & 0.000 \\
\hline $\begin{array}{l}\text { KRA०60 } \\
1\end{array}$ & 68.549 & $\odot . \odot \odot \odot$ & $\odot . \odot \odot \odot$ & 0.025 & $\odot . \odot \odot \odot$ \\
\hline $\begin{array}{l}\text { KRA०61 } \\
1\end{array}$ & 24.404 & 0.000 & 0.000 & 0.000 & 0.000 \\
\hline $\begin{array}{l}\text { KRA०62 } \\
1\end{array}$ & 1.287 & $\odot .000$ & 0.001 & $\odot .007$ & $\odot .00 \odot$ \\
\hline $\begin{array}{l}\text { KRA@63 } \\
1\end{array}$ & 56.500 & $\odot .0 \odot \odot$ & $\odot .0 \odot \odot$ & 0.001 & $\odot .0 \odot \odot$ \\
\hline $\begin{array}{l}\text { KRA@64 } \\
1\end{array}$ & 90.396 & $\odot .000$ & $\odot . \odot \odot \odot$ & 0.010 & 0.000 \\
\hline $\begin{array}{l}\text { KRA@65 } \\
1\end{array}$ & 38.438 & $\odot .00 \odot$ & $\odot .0 \odot \odot$ & 0.000 & 0.000 \\
\hline $\begin{array}{l}\text { KRA@66 } \\
1\end{array}$ & 93.046 & $\odot .00 \odot$ & $\odot .00 \odot$ & 0.002 & $\odot .0 \odot \odot$ \\
\hline $\begin{array}{l}\text { KRA@67 } \\
1\end{array}$ & 98.968 & $\odot .000$ & $\odot .000$ & 0.003 & $\odot .00 \odot$ \\
\hline $\begin{array}{l}\text { KRA@68 } \\
1\end{array}$ & 98.402 & $\odot .000$ & 0.000 & 0.006 & $\odot .00 \odot$ \\
\hline $\begin{array}{l}\text { KRA๑69 } \\
1\end{array}$ & 87.670 & $\odot .00 \odot$ & $\odot .000$ & 0.012 & $\odot .000$ \\
\hline $\begin{array}{l}\text { KRA๑70 } \\
1\end{array}$ & 8.454 & $\odot .000$ & $\odot .000$ & 0.001 & 0.000 \\
\hline $\begin{array}{l}\text { KRA๑71 } \\
1\end{array}$ & 61.602 & $\odot .00 \odot$ & $\odot .0 \odot \odot$ & 0.005 & 0.000 \\
\hline $\begin{array}{l}\text { KRA@72 } \\
1\end{array}$ & 56.537 & $\odot .0 \odot \odot$ & $\odot .0 \odot \odot$ & 0.001 & $\odot .000$ \\
\hline $\begin{array}{l}\text { KRA๑73 } \\
1\end{array}$ & 27.131 & $\odot .00 \odot$ & $\odot .000$ & 0.000 & $\odot .00 \odot$ \\
\hline $\begin{array}{l}\text { KRA๑74 } \\
1\end{array}$ & 57.904 & $\odot .000$ & $\odot .0 \odot \odot$ & 0.002 & 0.000 \\
\hline $\begin{array}{l}\text { KRA@75 } \\
1\end{array}$ & 40.140 & $\odot .000$ & 0.000 & 0.000 & $\odot .00 \odot$ \\
\hline
\end{tabular}




\begin{tabular}{|c|c|c|c|c|c|}
\hline $\begin{array}{l}\text { KRAO76 } \\
1\end{array}$ & 91.749 & $\odot . \odot \odot \odot$ & $\odot . \odot \odot \odot$ & $\odot .0 \odot 2$ & $\odot . \odot \odot \odot$ \\
\hline $\begin{array}{l}\text { KRA@77 } \\
1\end{array}$ & 41.725 & $\odot .00 \odot$ & 0.000 & $\odot .00 \odot$ & $\odot .00 \odot$ \\
\hline $\begin{array}{l}\text { KRA@78 } \\
1\end{array}$ & 21.031 & $\odot . \odot \odot \odot$ & $\odot . \odot \odot \odot$ & $\odot . \odot \odot \odot$ & $\odot . \odot \odot \odot$ \\
\hline $\begin{array}{l}\text { KRA@79 } \\
1\end{array}$ & 9.723 & 0.000 & 0.000 & $\odot .000$ & $0.00 \odot$ \\
\hline $\begin{array}{l}\text { KRA०80 } \\
1\end{array}$ & 0.452 & 0.000 & 0.001 & $\odot .001$ & $\odot .00 \odot$ \\
\hline $\begin{array}{l}\text { KRA๑81 } \\
1\end{array}$ & 25.570 & 0.000 & $\odot . \odot \odot \odot$ & ๑.033 & $\odot . \odot \odot \odot$ \\
\hline $\begin{array}{l}\text { KRA@82 } \\
1\end{array}$ & 92.196 & 0.000 & 0.000 & 0.002 & 0.000 \\
\hline $\begin{array}{l}\text { KRA083 } \\
1\end{array}$ & 38.040 & 0.000 & 0.000 & 0.042 & $\odot .00 \odot$ \\
\hline $\begin{array}{l}\text { KRA०84 } \\
1\end{array}$ & 84.597 & $\odot . \odot \odot \odot$ & $\odot .00 \odot$ & $\odot .0 \odot 2$ & $\odot .00 \odot$ \\
\hline $\begin{array}{l}\text { KRA०85 } \\
1\end{array}$ & 86.771 & $\odot . \odot \odot \odot$ & $\odot . \odot \odot \odot$ & ๑ . 012 & $\odot . \odot \odot \odot$ \\
\hline $\begin{array}{l}\text { KRA०86 } \\
1\end{array}$ & 25.541 & $\odot . \odot \odot \odot$ & $\odot . \odot \odot \odot$ & 0.060 & $\odot . \odot \odot \odot$ \\
\hline $\begin{array}{l}\text { KRA๑87 } \\
1\end{array}$ & 23.995 & $\odot . \odot \odot \odot$ & $\odot . \odot \odot \odot$ & $\odot .137$ & $\odot . \odot \odot \odot$ \\
\hline $\begin{array}{l}\text { KRA๑88 } \\
1\end{array}$ & 43.367 & $\odot .0 \odot \odot$ & $\odot .0 \odot \odot$ & $\odot .007$ & $\odot .0 \odot \odot$ \\
\hline $\begin{array}{l}\text { KRA०89 } \\
1\end{array}$ & 32.109 & $\odot . \odot \odot \odot$ & $\odot . \odot \odot \odot$ & $\odot .0 \odot 2$ & $\odot . \odot \odot \odot$ \\
\hline $\begin{array}{l}\text { KRA०9० } \\
1\end{array}$ & 23.014 & $\odot . \odot \odot \odot$ & $\odot . \odot \odot \odot$ & ๑.๑๑3 & $\odot .00 \odot$ \\
\hline $\begin{array}{l}\text { KRA०91 } \\
1\end{array}$ & 18.938 & $\odot .0 \odot \odot$ & $\odot .00 \odot$ & ๑ . 012 & $\odot . \odot \odot \odot$ \\
\hline $\begin{array}{l}\text { KRA@92 } \\
1\end{array}$ & 27.661 & $\odot . \odot \odot \odot$ & $\odot . \odot \odot \odot$ & $\odot .057$ & $\odot .00 \odot$ \\
\hline $\begin{array}{l}\text { KRA๑93 } \\
1\end{array}$ & 88.773 & 0.000 & $\odot .0 \odot \odot$ & $\odot .002$ & $\odot . \odot \odot \odot$ \\
\hline $\begin{array}{l}\text { KRA०95 } \\
1\end{array}$ & 77.407 & $\odot . \odot \odot \odot$ & $\odot . \odot \odot \odot$ & $\odot . \odot \odot 9$ & $\odot . \odot \odot \odot$ \\
\hline $\begin{array}{l}\text { KRA०96 } \\
1\end{array}$ & 70.041 & $\odot . \odot \odot \odot$ & $\odot . \odot \odot \odot$ & 0.001 & $\odot . \odot \odot \odot$ \\
\hline $\begin{array}{l}\text { KRA113 } \\
1\end{array}$ & 92.398 & 0.000 & $0.00 \odot$ & $\odot .0 \odot 2$ & $\odot .0 \odot \odot$ \\
\hline $\begin{array}{l}\text { KRA114 } \\
1\end{array}$ & 37.260 & $\odot . \odot \odot \odot$ & $\odot .0 \odot \odot$ & 0.014 & $\odot .0 \odot \odot$ \\
\hline $\begin{array}{l}\text { KRA115 } \\
1\end{array}$ & 46.743 & $\odot . \odot \odot \odot$ & $\odot .00 \odot$ & $\odot .039$ & $\odot . \odot \odot \odot$ \\
\hline $\begin{array}{l}\text { KRA116 } \\
1\end{array}$ & 72.873 & $\odot . \odot \odot \odot$ & $\odot .00 \odot$ & ๑. . 018 & $\odot . \odot \odot \odot$ \\
\hline $\begin{array}{l}\text { KRA117 } \\
1\end{array}$ & 69.651 & $\odot . \odot \odot \odot$ & $\odot . \odot \odot \odot$ & 0.011 & $\odot . \odot \odot \odot$ \\
\hline $\begin{array}{l}\text { KRA118 } \\
1\end{array}$ & 39.607 & $\odot . \odot \odot \odot$ & $\odot . \odot \odot \odot$ & $\odot .032$ & $\odot . \odot \odot \odot$ \\
\hline $\begin{array}{l}\text { KRA119 } \\
1\end{array}$ & 45.084 & $\odot . \odot \odot \odot$ & $\odot . \odot \odot \odot$ & $\odot .0 \odot 6$ & $\odot . \odot \odot \odot$ \\
\hline
\end{tabular}




\begin{tabular}{|c|c|c|c|c|c|}
\hline $\begin{array}{l}\text { KRA120 } \\
1\end{array}$ & 53.789 & $\odot . \odot \odot \odot$ & $\odot .00 \odot$ & $\odot .0 \odot 9$ & $\odot .0 \odot \odot$ \\
\hline $\begin{array}{l}\text { KRA121 } \\
1\end{array}$ & 48.201 & $\odot . \odot \odot \odot$ & $\odot .00 \odot$ & ๑. . 011 & $\odot .0 \odot \odot$ \\
\hline $\begin{array}{l}\text { KRA122 } \\
1\end{array}$ & $74.0 \odot 3$ & $\odot . \odot \odot \odot$ & $\odot . \odot \odot \odot$ & ๑. 005 & $\odot .0 \odot \odot$ \\
\hline $\begin{array}{l}\text { KRA123 } \\
1\end{array}$ & 31.154 & 0.000 & 0.000 & 0.021 & 0.000 \\
\hline $\begin{array}{l}\text { KRA126 } \\
1\end{array}$ & 71.070 & 0.000 & $\odot .000$ & 0.014 & 0.000 \\
\hline $\begin{array}{l}\text { KRA127 } \\
1\end{array}$ & 36.023 & 0.000 & $\odot .000$ & ๑. . 022 & 0.000 \\
\hline $\begin{array}{l}\text { KRA128 } \\
1\end{array}$ & 85.780 & 0.000 & 0.000 & 0.013 & 0.000 \\
\hline $\begin{array}{l}\text { KRA129 } \\
1\end{array}$ & 62.763 & 0.000 & 0.000 & 0.014 & 0.000 \\
\hline $\begin{array}{l}\text { KRA130 } \\
1\end{array}$ & 63.814 & 0.000 & $\odot .000$ & 0.004 & $\odot .0 \odot \odot$ \\
\hline $\begin{array}{l}\text { KRA131 } \\
1\end{array}$ & 56.672 & 0.000 & 0.000 & 0.013 & 0.000 \\
\hline $\begin{array}{l}\text { KRA132 } \\
1\end{array}$ & 80.473 & 0.000 & 0.000 & 0.015 & 0.000 \\
\hline $\begin{array}{l}\text { KRA133 } \\
1\end{array}$ & 83.404 & $\odot . \odot \odot \odot$ & $\odot . \odot \odot \odot$ & $\odot .0 \odot 4$ & 0.000 \\
\hline $\begin{array}{l}\text { KRA134 } \\
1\end{array}$ & 25.014 & $\odot . \odot \odot \odot$ & $\odot . \odot \odot \odot$ & 0.002 & 0.000 \\
\hline $\begin{array}{l}\text { KRA135 } \\
1\end{array}$ & 51.891 & 0.000 & 0.000 & $\odot .008$ & 0.000 \\
\hline $\begin{array}{l}\text { KRA136 } \\
1\end{array}$ & 87.877 & $\odot . \odot \odot \odot$ & $\odot . \odot \odot \odot$ & $\odot .0 \odot 7$ & 0.000 \\
\hline $\begin{array}{l}\text { KRA137 } \\
1\end{array}$ & 1.454 & $\odot . \odot \odot \odot$ & $\odot . \odot \odot \odot$ & 0.628 & $\odot .00 \odot$ \\
\hline $\begin{array}{l}\text { KRA139 } \\
1\end{array}$ & 54.483 & $\odot . \odot \odot \odot$ & $\odot . \odot \odot \odot$ & $\odot .0 \odot 3$ & $\odot .0 \odot \odot$ \\
\hline $\begin{array}{l}\text { KRA140 } \\
1\end{array}$ & 26.516 & $\odot . \odot \odot \odot$ & $\odot . \odot \odot \odot$ & ๑ . 010 & $\odot . \odot \odot \odot$ \\
\hline $\begin{array}{l}\text { KRA141 } \\
1\end{array}$ & 63.171 & 0.000 & $\odot . \odot \odot \odot$ & $\odot .004$ & 0.000 \\
\hline $\begin{array}{l}\text { KRA142 } \\
1\end{array}$ & 32.299 & $\odot . \odot \odot \odot$ & $\odot . \odot \odot \odot$ & $\odot .072$ & $\odot .0 \odot \odot$ \\
\hline $\begin{array}{l}\text { KRA143 } \\
1\end{array}$ & 42.724 & 0.000 & 0.000 & 0.006 & 0.000 \\
\hline $\begin{array}{l}\text { KRA144 } \\
1\end{array}$ & 58.220 & 0.000 & 0.000 & 0.005 & 0.000 \\
\hline $\begin{array}{l}\text { KRA145 } \\
1\end{array}$ & 34.490 & 0.000 & 0.000 & $\odot .008$ & 0.000 \\
\hline $\begin{array}{l}\text { KRA146 } \\
1\end{array}$ & 9.526 & 0.000 & $\odot .00 \odot$ & $\odot .000$ & 0.000 \\
\hline $\begin{array}{l}\text { KRA149 } \\
1\end{array}$ & 40.343 & 0.000 & 0.000 & 0.017 & 0.000 \\
\hline $\begin{array}{l}\text { KRA150 } \\
1\end{array}$ & 33.413 & 0.000 & 0.000 & $\odot .008$ & 0.000 \\
\hline $\begin{array}{l}\text { KRA152 } \\
1\end{array}$ & 50.214 & 0.000 & 0.000 & 0.025 & 0.000 \\
\hline
\end{tabular}




\begin{tabular}{|c|c|c|c|c|c|}
\hline $\begin{array}{l}\text { KRA153 } \\
1\end{array}$ & 68.266 & $\odot . \odot \odot \odot$ & $\odot . \odot \odot \odot$ & $\odot .003$ & $\odot .0 \odot \odot$ \\
\hline $\begin{array}{l}\text { KRA175 } \\
1\end{array}$ & 20.268 & $\odot .00 \odot$ & $\odot .000$ & 0.005 & 0.000 \\
\hline $\begin{array}{l}\text { KRA196 } \\
1\end{array}$ & 89.355 & $\odot .000$ & $\odot .00 \odot$ & 0.003 & 0.000 \\
\hline $\begin{array}{l}\text { KRA226 } \\
1\end{array}$ & 47.553 & $\odot .00 \odot$ & 0.000 & ๑. 017 & 0.000 \\
\hline $\begin{array}{l}\text { KRA228 } \\
1\end{array}$ & 11.927 & $\odot . \odot \odot \odot$ & 0.000 & ๑. 015 & 0.000 \\
\hline $\begin{array}{l}\text { KRA230 } \\
1\end{array}$ & 9.759 & $\odot .00 \odot$ & $\odot .00 \odot$ & $\odot .369$ & $\odot .0 \odot \odot$ \\
\hline $\begin{array}{l}\text { KRA236 } \\
1\end{array}$ & 22.747 & $\odot .00 \odot$ & 0.000 & 0.155 & 0.000 \\
\hline $\begin{array}{l}\text { KRA237 } \\
1\end{array}$ & 46.338 & $\odot .00 \odot$ & $\odot .000$ & $\odot .022$ & 0.000 \\
\hline $\begin{array}{l}\text { KRA239 } \\
1\end{array}$ & 14.403 & $\odot . \odot \odot \odot$ & $\odot .00 \odot$ & 0.177 & 0.000 \\
\hline $\begin{array}{l}\text { KRA241 } \\
1\end{array}$ & 20.975 & $\odot . \odot \odot \odot$ & $\odot .00 \odot$ & 0.163 & 0.000 \\
\hline
\end{tabular}

The following specimens are in the file EDALCA3

\begin{tabular}{|c|c|c|c|c|c|c|}
\hline & Probabi & ties: & & & & \\
\hline $\begin{array}{l}\text { ID. NO. } \\
\text { Into: }\end{array}$ & EDALCA1 & EDALCA3 & EDALCA4 & EDALCA5 & EDALCA7 & From: \\
\hline $\begin{array}{l}\text { KRA@10 } \\
2\end{array}$ & $\odot .000$ & 3.897 & 0.017 & $\odot .000$ & $\odot .000$ & 2 \\
\hline $\begin{array}{l}\text { KRA@11 } \\
2\end{array}$ & $\odot .0 \odot \odot$ & 1.923 & 0.020 & $\odot .000$ & 0.000 & 2 \\
\hline $\begin{array}{l}\text { KRA@12 } \\
2\end{array}$ & $\odot .0 \odot \odot$ & 5.020 & $\odot .004$ & 0.000 & 0.000 & 2 \\
\hline $\begin{array}{l}\text { KRA०13 } \\
2\end{array}$ & $\odot .00 \odot$ & 25.942 & 0.005 & 0.000 & 0.000 & 2 \\
\hline $\begin{array}{l}\text { KRA๑94 } \\
2\end{array}$ & $\odot .00 \odot$ & 0.358 & 0.001 & 0.000 & 0.000 & 2 \\
\hline $\begin{array}{l}\text { KRA124 } \\
2\end{array}$ & $\odot .0 \odot \odot$ & 91.505 & $\odot .0 \odot \odot$ & $\odot .0 \odot \odot$ & $\odot .0 \odot \odot$ & 2 \\
\hline $\begin{array}{l}\text { KRA125 } \\
2\end{array}$ & $\odot .0 \odot \odot$ & 99.830 & $\odot .00 \odot$ & $\odot .0 \odot \odot$ & $\odot . \odot \odot \odot$ & 2 \\
\hline $\begin{array}{l}\text { KRA138 } \\
2\end{array}$ & $\odot .0 \odot \odot$ & 2.696 & $\odot .0 \odot \odot$ & $\odot .0 \odot \odot$ & $\odot . \odot \odot \odot$ & 2 \\
\hline $\begin{array}{l}\text { KRA147 } \\
2\end{array}$ & $\odot .0 \odot \odot$ & 97.858 & $\odot .00 \odot$ & 0.000 & $\odot . \odot \odot \odot$ & 2 \\
\hline $\begin{array}{l}\text { KRA148 } \\
2\end{array}$ & $\odot . \odot \odot \odot$ & 92.288 & $0.00 \odot$ & $\odot . \odot \odot \odot$ & $\odot . \odot \odot \odot$ & 2 \\
\hline $\begin{array}{l}\text { KRA154 } \\
2\end{array}$ & 0.000 & 0.070 & $\odot .00 \odot$ & $0.00 \odot$ & $\odot .00 \odot$ & 2 \\
\hline $\begin{array}{l}\text { KRA155 } \\
2\end{array}$ & $\odot .0 \odot \odot$ & 95.461 & $\odot .00 \odot$ & $0.00 \odot$ & $\odot . \odot \odot \odot$ & 2 \\
\hline $\begin{array}{l}\text { KRA156 } \\
2\end{array}$ & $\odot .0 \odot \odot$ & 44.626 & 0.002 & 0.000 & 0.000 & 2 \\
\hline RA157 & $\odot . \odot \odot \odot$ & 83.668 & $\odot .0 \odot \odot$ & $\odot .0 \odot \odot$ & $\odot .00 \odot$ & 2 \\
\hline
\end{tabular}




\begin{tabular}{|c|c|c|c|c|c|}
\hline $\begin{array}{l}\text { KRA158 } \\
2\end{array}$ & $\odot . ๑ \odot \odot$ & 63.999 & $\odot .001$ & $\odot .0 \odot \odot$ & $\odot . \odot \odot \odot$ \\
\hline $\begin{array}{l}\text { KRA159 } \\
2\end{array}$ & $\odot . \odot \odot \odot$ & 86.648 & $\odot .00 \odot$ & $\odot .000$ & 0.000 \\
\hline $\begin{array}{l}\text { KRA160 } \\
2\end{array}$ & $\odot . \odot \odot \odot$ & 66.778 & $\odot . \odot \odot \odot$ & $\odot . \odot \odot \odot$ & $\odot . \odot \odot \odot$ \\
\hline $\begin{array}{l}\text { KRA161 } \\
2\end{array}$ & $\odot . ๑ \odot \odot$ & 99.420 & $\odot .00 \odot$ & $\odot .000$ & 0.000 \\
\hline $\begin{array}{l}\text { KRA162 } \\
2\end{array}$ & $\odot . \odot \odot \odot$ & 96.154 & $\odot . \odot \odot \odot$ & $\odot .0 \odot \odot$ & 0.000 \\
\hline $\begin{array}{l}\text { KRA163 } \\
2\end{array}$ & $\odot .00 \odot$ & 96.306 & $\odot . \odot \odot \odot$ & $\odot .0 \odot \odot$ & $\odot .0 \odot \odot$ \\
\hline $\begin{array}{l}\text { KRA164 } \\
2\end{array}$ & $\odot .00 \odot$ & 66.605 & $\odot .0 \odot \odot$ & $\odot .000$ & 0.000 \\
\hline $\begin{array}{l}\text { KRA165 } \\
2\end{array}$ & $\odot .00 \odot$ & 97.886 & $\odot .00 \odot$ & $\odot .000$ & 0.000 \\
\hline $\begin{array}{l}\text { KRA166 } \\
2\end{array}$ & $\odot . \odot \odot \odot$ & 93.661 & $\odot .0 \odot \odot$ & $\odot .000$ & 0.000 \\
\hline $\begin{array}{l}\text { KRA167 } \\
2\end{array}$ & $\odot . \odot \odot \odot$ & 85.150 & 0.000 & 0.000 & 0.000 \\
\hline $\begin{array}{l}\text { KRA168 } \\
2\end{array}$ & $\odot .00 \odot$ & 80.967 & $\odot .0 \odot \odot$ & $\odot .000$ & 0.000 \\
\hline $\begin{array}{l}\text { KRA169 } \\
2\end{array}$ & $\odot . \odot \odot \odot$ & 99.790 & $\odot . \odot \odot \odot$ & $\odot . \odot \odot \odot$ & $\odot . \odot \odot \odot$ \\
\hline $\begin{array}{l}\text { KRA170 } \\
2\end{array}$ & $\odot . \odot \odot \odot$ & 61.679 & $\odot . \odot \odot \odot$ & $\odot .00 \odot$ & $\odot .00 \odot$ \\
\hline $\begin{array}{l}\text { KRA171 } \\
2\end{array}$ & $\odot .00 \odot$ & 90.101 & $\odot . \odot \odot \odot$ & $\odot .000$ & $\odot .000$ \\
\hline $\begin{array}{l}\text { KRA172 } \\
2\end{array}$ & $\odot . \odot \odot \odot$ & 57.630 & $\odot . \odot \odot \odot$ & $\odot .0 \odot \odot$ & $\odot .0 \odot \odot$ \\
\hline $\begin{array}{l}\text { KRA173 } \\
2\end{array}$ & $\odot . \odot \odot \odot$ & 97.570 & $\odot . \odot \odot \odot$ & $\odot . \odot \odot \odot$ & $\odot . \odot \odot \odot$ \\
\hline
\end{tabular}

The following specimens are in the file EDALCA4 Probabilities:

\begin{tabular}{|c|c|c|c|c|c|c|}
\hline $\begin{array}{l}\text { ID. NO. } \\
\text { Into: }\end{array}$ & EDALCA1 & EDALCA3 & EDALCA4 & EDALCA5 & EDALCA7 & From: \\
\hline $\begin{array}{l}\text { KRA०97 } \\
3\end{array}$ & $\odot .0 \odot \odot$ & 0.001 & 28.556 & 0.000 & $\odot .000$ & 3 \\
\hline $\begin{array}{l}\text { KRA०98 } \\
3\end{array}$ & $\odot . \odot \odot \odot$ & $\odot .0 \odot \odot$ & 15.666 & 0.000 & $\odot . \odot \odot \odot$ & 3 \\
\hline $\begin{array}{l}\text { KRA099 } \\
3\end{array}$ & $\odot . \odot \odot \odot$ & $\odot .0 \odot 2$ & 88.060 & $\odot . \odot \odot \odot$ & $\odot . \odot \odot \odot$ & 3 \\
\hline $\begin{array}{l}\text { KRA100 } \\
3\end{array}$ & $\odot .000$ & $\odot .0 \odot 2$ & 76.932 & 0.000 & $\odot .000$ & 3 \\
\hline $\begin{array}{l}\text { KRA103 } \\
3\end{array}$ & $\odot .000$ & $\odot .000$ & 14.160 & 0.000 & $\odot .000$ & 3 \\
\hline $\begin{array}{l}\text { KRA221 } \\
3\end{array}$ & $\odot .0 \odot \odot$ & 0.008 & 29.897 & 0.000 & $\odot .000$ & 3 \\
\hline $\begin{array}{l}\text { KRA242 } \\
3\end{array}$ & $\odot .0 \odot \odot$ & 0.001 & 17.735 & 0.000 & $\odot .000$ & 3 \\
\hline KRA253 & $\odot .0 \odot \odot$ & 0.006 & 48.916 & $\odot .00 \odot$ & $\odot .0 \odot \odot$ & 3 \\
\hline
\end{tabular}

3 


$\begin{array}{lcccccr}\text { KRA254 } & 0.000 & 0.006 & 67.107 & 0.000 & 0.000 & 3 \\ 3 & 0.00 \odot & 0.008 & 33.895 & 0.00 \odot & 0.00 \odot & 3 \\ \text { KRA255 } & 0.00 & & \\ 3 & & & & & \\ \text { KRA256 } & 0.000 & 0.019 & 31.273 & 0.000 & 0.000 & 3 \\ 3 & & & & & \\ \text { KRA257 } & 0.00 \odot & 0.007 & 73.447 & 0.00 \odot & 0.00 \odot & 3 \\ 3 & & & & & \\ \text { KRA258 } & 0.00 \odot & 0.007 & 74.720 & 0.00 \odot & 0.00 \odot & 3 \\ 3 & & & & & & \end{array}$

The following specimens are in the file EDALCA5 Probabilities:

ID. No

KRA104

4

KRA105

4

KRA106

$$
4
$$

KRA107

4

KRA108

4

KRA109

4

KRA110

4

KRA111

4

KRA112

4

KRA174

KRA176

4

KRA190

4

KRA209

5

KRA210

4

KRA211

4

KRA212

4

KRA213

4

KRA214

4

KRA215

4
EDALCA1 EDALCA3

$0.00 \odot \quad 0.000$

EDALCA

$\odot .007$

๑. 017

0.029

8.137

$\odot .002$

33.951

$\odot .003$

45.450

0.001

42.405

$\odot .001$

22.776

$\odot .001$

1.343

$\odot .002$

2.532

$\odot .003$

47.961

$\odot . \odot \odot \odot$

42.496

$\odot .000$

54.034

$\odot .000$

0.130

$\odot .000$

76.751

$\odot . \odot \odot \odot$

26.192

$\odot . \odot \odot \odot$

81.904

$\odot .001$

98.548

$0.000 \quad 79.557$

$\odot .000$

0.000

0.000

$\odot .0 \odot \odot$

0.000

$\odot .000 \quad 25.704$

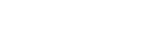




\begin{tabular}{|c|c|c|c|c|c|}
\hline $\begin{array}{l}\text { KRA217 } \\
4\end{array}$ & $\odot . ๑ \odot \odot$ & $\odot . \odot \odot \odot$ & $\odot . \odot \odot \odot$ & 54.951 & $\odot .001$ \\
\hline $\begin{array}{l}\text { KRA218 } \\
4\end{array}$ & $\odot . ๑ \odot \odot$ & $\odot . ๑ \odot \odot$ & $\odot . \odot \odot \odot$ & 82.989 & $\odot . \odot \odot \odot$ \\
\hline $\begin{array}{l}\text { KRA219 } \\
4\end{array}$ & $\odot . \odot \odot \odot$ & $\odot . \odot \odot \odot$ & $\odot . \odot \odot \odot$ & 72.881 & $\odot . \odot \odot \odot$ \\
\hline $\begin{array}{l}\text { KRA220 } \\
4\end{array}$ & $\odot . ๑ \odot \odot$ & $\odot . \odot \odot \odot$ & $\odot .0 \odot 2$ & 81.408 & 0.000 \\
\hline $\begin{array}{l}\text { KRA222 } \\
4\end{array}$ & $\odot . \odot \odot \odot$ & $\odot . \odot \odot \odot$ & $\odot .001$ & 89.515 & 0.000 \\
\hline $\begin{array}{l}\text { KRA223 } \\
4\end{array}$ & $\odot . ๑ \odot \odot$ & $\odot . \odot \odot \odot$ & $\odot .0 \odot \odot$ & 78.566 & 0.000 \\
\hline $\begin{array}{l}\text { KRA224 } \\
4\end{array}$ & $\odot .00 \odot$ & $\odot .00 \odot$ & 0.001 & 92.656 & 0.000 \\
\hline $\begin{array}{l}\text { KRA225 } \\
4\end{array}$ & $\odot .00 \odot$ & 0.001 & 0.006 & 8.668 & 0.000 \\
\hline $\begin{array}{l}\text { KRA227 } \\
4\end{array}$ & $\odot . \odot \odot \odot$ & $\odot . \odot \odot \odot$ & $\odot .0 \odot 2$ & 46.614 & 0.000 \\
\hline $\begin{array}{l}\text { KRA229 } \\
4\end{array}$ & $\odot .00 \odot$ & $\odot .00 \odot$ & $\odot .000$ & 66.279 & $\odot .001$ \\
\hline $\begin{array}{l}\text { KRA231 } \\
4\end{array}$ & $\odot .00 \odot$ & $\odot .00 \odot$ & 0.001 & 45.370 & 0.000 \\
\hline $\begin{array}{l}\text { KRA232 } \\
4\end{array}$ & $\odot . \odot \odot \odot$ & $\odot . \odot \odot \odot$ & $\odot . \odot \odot 2$ & 81.929 & $\odot . \odot \odot \odot$ \\
\hline $\begin{array}{l}\text { KRA233 } \\
4\end{array}$ & $\odot . \odot \odot \odot$ & $\odot . \odot \odot 1$ & 0.010 & 13.989 & $\odot .00 \odot$ \\
\hline $\begin{array}{l}\text { KRA234 } \\
4\end{array}$ & $\odot . \odot \odot \odot$ & $\odot .00 \odot$ & 0.001 & 96.584 & $\odot .00 \odot$ \\
\hline $\begin{array}{l}\text { KRA235 } \\
4\end{array}$ & $\odot . ๑ \odot \odot$ & $\odot . \odot \odot 1$ & $\odot .003$ & 29.751 & $\odot .00 \odot$ \\
\hline $\begin{array}{l}\text { KRA240 } \\
4\end{array}$ & $\odot . \odot \odot \odot$ & $\odot . \odot \odot \odot$ & $\odot . \odot \odot 1$ & 95.696 & $\odot . \odot \odot \odot$ \\
\hline $\begin{array}{l}\text { KRA244 } \\
4\end{array}$ & $\odot . \odot \odot \odot$ & $\odot . \odot \odot 1$ & $\odot . \odot \odot 4$ & 20.670 & $\odot .0 \odot \odot$ \\
\hline $\begin{array}{l}\text { KRA246 } \\
4\end{array}$ & $\odot . \odot \odot \odot$ & $\odot . \odot \odot \odot$ & $\odot . \odot \odot \odot$ & 59.534 & $\odot .0 \odot 2$ \\
\hline $\begin{array}{l}\text { KRA247 } \\
4\end{array}$ & $\odot . \odot \odot \odot$ & $\odot . \odot \odot \odot$ & $\odot .001$ & 97.982 & 0.000 \\
\hline $\begin{array}{l}\text { KRA248 } \\
4\end{array}$ & $\odot . \odot \odot \odot$ & $\odot . \odot \odot \odot$ & $\odot .001$ & 98.900 & $\odot .00 \odot$ \\
\hline $\begin{array}{l}\text { KRA249 } \\
4\end{array}$ & $\odot . \odot \odot \odot$ & $\odot . \odot \odot \odot$ & 0.001 & 85.560 & $\odot .000$ \\
\hline $\begin{array}{l}\text { KRA250 } \\
4\end{array}$ & $\odot .00 \odot$ & $\odot . \odot \odot \odot$ & $\odot .002$ & 90.975 & 0.000 \\
\hline $\begin{array}{l}\text { KRA251 } \\
4\end{array}$ & $\odot .00 \odot$ & $\odot .00 \odot$ & 0.000 & 75.418 & 0.001 \\
\hline
\end{tabular}

The following specimens are in the file EDALCA7 Probabilities:

ID. NO. EDALCA1 EDALCA3 EDALCA4 EDALCA5 EDALCA7 From: Into:

$\begin{array}{lllllll}\text { KRA101 } & \odot .0 \odot \odot ~ & \odot . \odot \odot \odot ~ & \odot .0 \odot \odot & \odot .038 & 10.599 & 5\end{array}$




\begin{tabular}{|c|c|c|c|c|c|}
\hline $\begin{array}{l}\text { KRA102 } \\
5\end{array}$ & $\odot . \odot \odot \odot$ & $\odot .0 \odot \odot$ & $\odot .00 \odot$ & 0.035 & 11.324 \\
\hline $\begin{array}{l}\text { KRA177 } \\
5\end{array}$ & $\odot . \odot \odot \odot$ & $\odot .0 \odot \odot$ & $\odot . \odot \odot \odot$ & $\odot .0 \odot \odot$ & 29.390 \\
\hline $\begin{array}{l}\text { KRA178 } \\
5\end{array}$ & $\odot . \odot \odot \odot$ & $\odot .0 \odot \odot$ & $\odot . \odot \odot \odot$ & $\odot . \odot \odot \odot$ & 82.237 \\
\hline $\begin{array}{l}\text { KRA179 } \\
5\end{array}$ & $\odot . \odot \odot \odot$ & $\odot .0 \odot \odot$ & $\odot . \odot \odot \odot$ & $0.00 \odot$ & 73.336 \\
\hline $\begin{array}{l}\text { KRA180 } \\
5\end{array}$ & $\odot .00 \odot$ & $\odot .0 \odot \odot$ & $\odot .0 \odot \odot$ & 0.000 & 46.784 \\
\hline $\begin{array}{l}\text { KRA181 } \\
5\end{array}$ & $\odot . \odot \odot \odot$ & $\odot . \odot \odot \odot$ & $\odot . \odot \odot \odot$ & $\odot . \odot \odot \odot$ & 66.087 \\
\hline $\begin{array}{l}\text { KRA182 } \\
5\end{array}$ & $\odot . \odot \odot \odot$ & $\odot .0 \odot \odot$ & $\odot .000$ & 0.000 & 94.872 \\
\hline $\begin{array}{l}\text { KRA183 } \\
5\end{array}$ & $\odot . \odot \odot \odot$ & 0.000 & $\odot .0 \odot \odot$ & $0.00 \odot$ & 67.497 \\
\hline $\begin{array}{l}\text { KRA184 } \\
5\end{array}$ & $\odot . ๑ \odot \odot$ & $\odot . \odot \odot \odot$ & $\odot . ๑ \odot \odot$ & $\odot . \odot \odot \odot$ & 89.225 \\
\hline $\begin{array}{l}\text { KRA185 } \\
5\end{array}$ & $\odot . ๑ \odot \odot$ & $\odot . \odot \odot \odot$ & $\odot . ๑ \odot \odot$ & $\odot . \odot \odot \odot$ & 69.610 \\
\hline $\begin{array}{l}\text { KRA186 } \\
5\end{array}$ & $\odot . ๑ \odot \odot$ & $\odot . \odot \odot \odot$ & $\odot . ๑ \odot \odot$ & 0.042 & 10.678 \\
\hline $\begin{array}{l}\text { KRA187 } \\
5\end{array}$ & $\odot . \odot \odot \odot$ & $\odot .0 \odot \odot$ & $\odot . \odot \odot \odot$ & $\odot .0 \odot \odot$ & 91.859 \\
\hline $\begin{array}{l}\text { KRA188 } \\
5\end{array}$ & $\odot . \odot \odot \odot$ & $\odot .0 \odot \odot$ & $\odot . \odot \odot \odot$ & 0.001 & 88.280 \\
\hline $\begin{array}{l}\text { KRA189 } \\
5\end{array}$ & $\odot . \odot \odot \odot$ & $\odot . \odot \odot \odot$ & $\odot .00 \odot$ & $\odot . \odot \odot 1$ & 69.136 \\
\hline $\begin{array}{l}\text { KRA191 } \\
5\end{array}$ & $\odot . \odot \odot \odot$ & $\odot .0 \odot \odot$ & $\odot .000$ & $\odot . \odot \odot \odot$ & 41.037 \\
\hline $\begin{array}{l}\text { KRA192 } \\
5\end{array}$ & $\odot . ๑ \odot \odot$ & $\odot . \odot \odot \odot$ & $\odot . ๑ \odot \odot$ & $\odot . \odot \odot \odot$ & 66.697 \\
\hline $\begin{array}{l}\text { KRA193 } \\
5\end{array}$ & $\odot . \odot \odot \odot$ & $\odot . \odot \odot \odot$ & $\odot . \odot \odot \odot$ & $\odot . \odot \odot \odot$ & 66.638 \\
\hline $\begin{array}{l}\text { KRA195 } \\
5\end{array}$ & $\odot . \odot \odot \odot$ & $\odot .0 \odot \odot$ & $\odot .000$ & $0.00 \odot$ & 85.100 \\
\hline $\begin{array}{l}\text { KRA197 } \\
5\end{array}$ & $\odot .00 \odot$ & $\odot .0 \odot \odot$ & $\odot .0 \odot \odot$ & $0.00 \odot$ & 91.463 \\
\hline $\begin{array}{l}\text { KRA198 } \\
5\end{array}$ & $\odot . \odot \odot \odot$ & $\odot .0 \odot \odot$ & $\odot . \odot \odot \odot$ & $0.00 \odot$ & 92.956 \\
\hline $\begin{array}{l}\text { KRA199 } \\
5\end{array}$ & $\odot . \odot \odot \odot$ & 0.000 & $\odot .000$ & $\odot . \odot \odot \odot$ & 4.290 \\
\hline $\begin{array}{l}\text { KRA200 } \\
5\end{array}$ & $\odot . \odot \odot \odot$ & $\odot .0 \odot \odot$ & $\odot .000$ & $\odot .0 \odot \odot$ & 59.283 \\
\hline $\begin{array}{l}\text { KRA201 } \\
5\end{array}$ & $\odot . \odot \odot \odot$ & $\odot .0 \odot \odot$ & $\odot .0 \odot \odot$ & 0.000 & 97.276 \\
\hline $\begin{array}{l}\text { KRA202 } \\
5\end{array}$ & $\odot . \odot \odot \odot$ & $\odot . \odot \odot \odot$ & $\odot . \odot \odot \odot$ & $\odot . \odot \odot \odot$ & 6.602 \\
\hline $\begin{array}{l}\text { KRA203 } \\
5\end{array}$ & $\odot .00 \odot$ & 0.000 & $\odot .000$ & 0.000 & 23.486 \\
\hline $\begin{array}{l}\text { KRA204 } \\
5\end{array}$ & 0.000 & $\odot .0 \odot \odot$ & $\odot .000$ & 0.001 & 43.584 \\
\hline $\begin{array}{l}\text { KRA205 } \\
5\end{array}$ & $\odot . ๑ \odot \odot$ & $\odot . \odot \odot \odot$ & $\odot . \odot \odot \odot$ & $\odot . \odot \odot \odot$ & $81.0 \odot 8$ \\
\hline
\end{tabular}




$\begin{array}{llllccr}\text { KRA207 } & 0.00 \odot & 0.00 \odot & 0.00 \odot & 0.000 & 81.443 & 5 \\ 5 & 0.000 & 0.00 \odot & 0.000 & 0.002 & 1.992 & 5 \\ \text { KRA238 } & 0.00 \odot & 0.00 \odot & 0.00 \odot & 0.00 \odot & 0.355 & 5 \\ 5 & & & & & \end{array}$

Summary of Classification Success:

Into:

$\begin{array}{lrrrrrr}\text { From: } & \text { EDALCA1 } & \text { EDALCA3 } & \text { EDALCA4 } & \text { EDALCA5 } & \text { EDALCA7 } & \text { Total } \\ \text { EDALCA1 } & 133 & \odot & 0 & 0 & 0 & 133 \\ \text { EDALCA3 } & \odot & 30 & \odot & 0 & 0 & 30 \\ \text { EDALCA4 } & 0 & 0 & 13 & 0 & 0 & 13 \\ \text { EDALCA5 } & 0 & 0 & 0 & 41 & 1 & 42 \\ \text { EDALCA7 } & \odot & 0 & 0 & 0 & 31 & 31 \\ \text { Total } & 133 & 30 & 13 & 41 & 32 & 249\end{array}$


Table DR8. Mahalanobis distance results for NAA

MAHALANOBIS DISTANCE CALCULATION AND POSTERIOR CLASSIFICATION

FOR TWO OR MORE GROUPS.

Date: $10 / 20 / 12$

File: Maha5.txt

Groups are:
1 ALCA1NAA
2 ALCA3NAA
3 ALCA4NAA
4 ALCA5NAA

Variables used:

Eu Th

Probabilities are jackknifed for specimens included in each group.

The following specimens are in the file ALCA1NAA

Probabilities:

\begin{tabular}{|c|c|c|c|c|c|c|}
\hline ID. NO. & ALCA1NAA & ALCA3NAA & ALCA4NAA & ALCA5NAA & From: & Into: \\
\hline KRA००1 & 78.933 & 0.015 & $\odot .00 \odot$ & $\odot .004$ & 1 & 1 \\
\hline 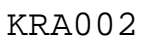 & 67.607 & 0.016 & $\odot .0 \odot \odot$ & $\odot .005$ & 1 & 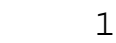 \\
\hline KRA०95 & 1.694 & 0.011 & $\odot . \odot \odot \odot$ & $\odot .001$ & 1 & \\
\hline KRA@38 & 57.491 & 0.017 & $\odot .0 \odot \odot$ & $\odot .007$ & 1 & \\
\hline KRA๑41 & 66.439 & 0.016 & $\odot .0 \odot \odot$ & $\odot .006$ & 1 & \\
\hline KRA๑46 & 45.878 & 0.012 & $\odot . \odot \odot \odot$ & $\odot .001$ & 1 & \\
\hline KRA๑62 & 98.870 & 0.015 & $\odot . \odot \odot \odot$ & $\odot .003$ & 1 & \\
\hline KRA๑7๑ & 23.979 & 0.016 & $\odot . \odot \odot \odot$ & 0.006 & 1 & \\
\hline KRA๑8๑ & $9 \odot .673$ & 0.013 & $\odot . \odot \odot \odot$ & $\odot . \odot \odot 2$ & 1 & \\
\hline KRA091 & 6.555 & 0.014 & $\odot . \odot \odot \odot$ & $\odot .0 \odot 2$ & 1 & \\
\hline
\end{tabular}

The following specimens are in the file ALCA3NAA Probabilities:

ID. NO. ALCA1NAA ALCA3NAA

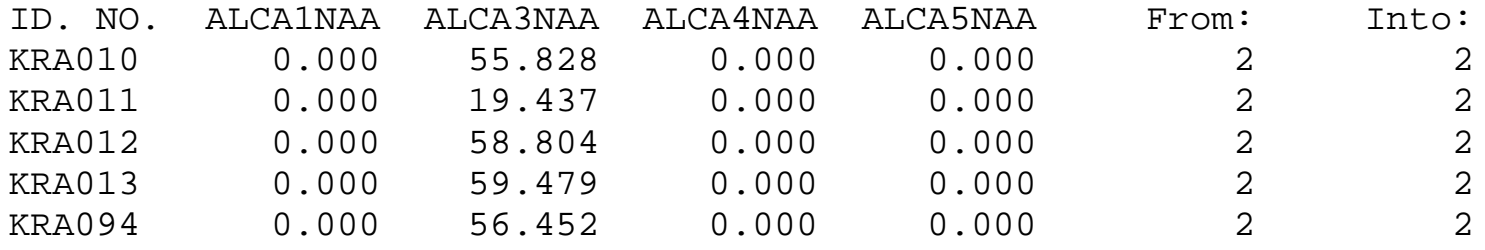

The following specimens are in the file ALCA4NAA Probabilities:

\begin{tabular}{|c|c|c|c|c|c|c|}
\hline ID. NO. & ALCA1NAA & ALCA3NAA & ALCA4NAA & ALCA5NAA & From: & Into: \\
\hline KRA- $\odot 97$ & $\odot .0 \odot \odot$ & $\odot .0 \odot 2$ & 21.454 & $\odot . \odot \odot \odot$ & 3 & 3 \\
\hline KRA๑98 & $\odot . \odot \odot \odot$ & $\odot . \odot \odot 2$ & 41.947 & $\odot . \odot \odot \odot$ & 3 & \\
\hline RA०99 & $\odot . \odot \odot \odot$ & $\odot . \odot \odot 2$ & 7.576 & $\odot . \odot \odot \odot$ & 3 & \\
\hline RA10० & $\odot . \odot \odot \odot$ & $\odot . \odot \odot 2$ & 99.986 & $\odot . \odot \odot \odot$ & 3 & \\
\hline RA103 & $\odot .000$ & $\odot .0 \odot 2$ & 79.037 & $\odot .0 \odot \odot$ & 3 & \\
\hline
\end{tabular}

The following specimens are in the file ALCA5NAA Probabilities:

ID. NO. ALCA1NAA ALCA3NAA ALCA4NAA ALCA5NAA From: Into: 


\begin{tabular}{|c|c|c|c|c|c|}
\hline KRA104 & $\odot . \odot \odot 4$ & $\odot .056$ & 0.000 & 82.246 & 4 \\
\hline KRA105 & $\odot .0 \odot 1$ & $\odot .053$ & $\odot . \odot \odot \odot$ & 17.968 & 4 \\
\hline KRA106 & ๑. .015 & $\odot .057$ & $\odot . \odot \odot \odot$ & 85.945 & 4 \\
\hline KRA107 & ๑. 011 & 0.044 & $\odot . \odot \odot \odot$ & 13.287 & 4 \\
\hline KRA108 & $\odot .003$ & 0.068 & $\odot .00 \odot$ & 26.716 & 4 \\
\hline KRA109 & $\odot .007$ & 0.064 & $\odot .00 \odot$ & 71.294 & 4 \\
\hline KRA110 & 0.037 & 0.051 & $\odot .00 \odot$ & 34.441 & 4 \\
\hline KRA111 & 0.011 & 0.051 & $\odot . \odot \odot \odot$ & 82.515 & 4 \\
\hline KRA112 & $\odot .020$ & 0.063 & $\odot .00 \odot$ & 22.859 & 4 \\
\hline \multicolumn{6}{|c|}{$\begin{array}{c}\text { Summary of Classification Success: } \\
\text { Into: }\end{array}$} \\
\hline From: & ALCA1NAA & ALCA3NAA & ALCA4NAA & ALCA5NAA & Total \\
\hline ALCA1NAA & 10 & $\Theta$ & $\Theta$ & 0 & 10 \\
\hline ALCA3NAA & $\odot$ & 5 & $\odot$ & 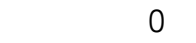 & 5 \\
\hline ALCA4NAA & $\odot$ & $\odot$ & 5 & $\odot$ & 5 \\
\hline ALCA5NAA & $\odot$ & $\odot$ & $\odot$ & 9 & 9 \\
\hline Total & 10 & 5 & 5 & 9 & 29 \\
\hline
\end{tabular}


Table DR9. Mahalanobis distance results for WD-XRF

MAHALANOBIS DISTANCE CALCULATION AND POSTERIOR CLASSIFICATION

FOR TWO OR MORE GROUPS.

Date: $10 / 21 / 12$

File: Maha7.txt

Groups are:
$1 \quad$ ALCA1V2
2 ALCA3V2
$3 \quad$ ALCA4V2
$4 \quad$ ALCA5V2
$5 \quad$ ALCA7V2

Variables used:

$$
\text { TiO2 Sr }
$$

Probabilities are jackknifed for specimens included in each group.

The following specimens are in the file ALCA1V2

\begin{tabular}{|c|c|c|c|c|c|c|}
\hline \multirow{2}{*}{$\begin{array}{l}\text { ID. NO. } \\
\text { Into: }\end{array}$} & \multicolumn{2}{|c|}{ Probabilities: } & \multirow[b]{2}{*}{ ALCA4V2 } & \multirow[b]{2}{*}{ ALCA5V2 } & \multirow[b]{2}{*}{ ALCA7V2 } & \multirow[b]{2}{*}{ From: } \\
\hline & ALCA1V2 & ALCA3V2 & & & & \\
\hline $\begin{array}{l}\text { KRA००1 } \\
1\end{array}$ & 95.110 & $\odot .0 \odot \odot$ & $\odot .0 \odot 2$ & $\odot .0 \odot \odot$ & $\odot . \odot \odot \odot$ & 1 \\
\hline $\begin{array}{l}\text { KRA००2 } \\
1\end{array}$ & 42.743 & $\odot . \odot \odot \odot$ & $\odot . \odot \odot 2$ & $\odot .0 \odot \odot$ & $\odot . \odot \odot \odot$ & 1 \\
\hline $\begin{array}{l}\text { KRA@26 } \\
1\end{array}$ & 83.678 & $\odot . \odot \odot \odot$ & $\odot . \odot \odot 2$ & $\odot .0 \odot \odot$ & $\odot . \odot \odot \odot$ & 1 \\
\hline $\begin{array}{l}\text { KRA०42 } \\
1\end{array}$ & 89.695 & $\odot . \odot \odot \odot$ & $\odot .002$ & 0.000 & $\odot .00 \odot$ & 1 \\
\hline $\begin{array}{l}\text { KRA058 } \\
1\end{array}$ & 19.643 & 0.000 & $\odot .002$ & 0.000 & $\odot .00 \odot$ & 1 \\
\hline $\begin{array}{l}\text { KRA०61 } \\
1\end{array}$ & 89.498 & $0.00 \odot$ & $\odot .002$ & $\odot . \odot \odot \odot$ & $\odot .00 \odot$ & 1 \\
\hline $\begin{array}{l}\text { KRA065 } \\
1\end{array}$ & 70.836 & 0.000 & $\odot .002$ & $\odot .00 \odot$ & $\odot .00 \odot$ & 1 \\
\hline $\begin{array}{l}\text { KRA068 } \\
1\end{array}$ & 10.471 & 0.000 & $\odot .0 \odot 2$ & $\odot .00 \odot$ & $\odot .0 \odot \odot$ & 1 \\
\hline $\begin{array}{l}\text { KRA०91 } \\
1\end{array}$ & 76.674 & 0.000 & $\odot .0 \odot 2$ & 0.000 & $\odot .00 \odot$ & 1 \\
\hline $\begin{array}{l}\text { KRA๑92 } \\
1\end{array}$ & 29.585 & 0.000 & $\odot .002$ & $\odot . \odot \odot \odot$ & $\odot . \odot \odot \odot$ & 1 \\
\hline $\begin{array}{l}\text { KRA०93 } \\
1\end{array}$ & 13.790 & 0.000 & $\odot .002$ & $\odot .000$ & $\odot . \odot \odot \odot$ & 1 \\
\hline $\begin{array}{l}\text { KRA095 } \\
1\end{array}$ & 70.516 & $\odot .0 \odot \odot$ & $\odot . \odot \odot 2$ & $\odot .0 \odot \odot$ & $\odot . \odot \odot \odot$ & 1 \\
\hline $\begin{array}{l}\text { KRA๑96 } \\
1\end{array}$ & 35.321 & 0.000 & 0.003 & 0.000 & $\odot .000$ & 1 \\
\hline $\begin{array}{l}\text { KRA113 } \\
1\end{array}$ & 88.258 & $\odot .0 \odot \odot$ & $\odot .002$ & 0.000 & $\odot . \odot \odot \odot$ & 1 \\
\hline KRA226 & 39.478 & 0.000 & $\odot .003$ & 0.000 & $\odot .00 \odot$ & 1 \\
\hline
\end{tabular}

1 


\begin{tabular}{|c|c|c|c|c|c|}
\hline $\begin{array}{l}\text { KRA228 } \\
1\end{array}$ & 16.065 & $\odot . \odot \odot \odot$ & $\odot .003$ & $\odot .0 \odot \odot$ & $\odot .0 \odot \odot$ \\
\hline $\begin{array}{l}\text { KRA230 } \\
1\end{array}$ & 17.834 & $\odot .000$ & $\odot .003$ & $\odot .000$ & 0.000 \\
\hline $\begin{array}{l}\text { M०4०72 } \\
1\end{array}$ & 27.141 & $\odot . \odot \odot \odot$ & $\odot . \odot \odot 2$ & $\odot . \odot \odot \odot$ & $\odot .0 \odot \odot$ \\
\hline $\begin{array}{l}\text { M04074 } \\
1\end{array}$ & 9.907 & $\odot . \odot \odot \odot$ & $\odot .0 \odot 2$ & $\odot .0 \odot \odot$ & $\odot .0 \odot \odot$ \\
\hline
\end{tabular}

The following specimens are in the file ALCA3V2

\begin{tabular}{|c|c|c|c|c|c|c|}
\hline & Probabj & ties: & & & & \\
\hline $\begin{array}{l}\text { ID. NO. } \\
\text { Into: }\end{array}$ & ALCA1V2 & ALCA3V2 & ALCA4V2 & ALCA5V2 & ALCA7V2 & From: \\
\hline $\begin{array}{l}\text { KRA@10 } \\
2\end{array}$ & 0.000 & 68.211 & $\odot .0 \odot 2$ & 0.000 & 0.000 & 2 \\
\hline $\begin{array}{l}\text { KRAO11 } \\
2\end{array}$ & $\odot .000$ & 42.554 & $\odot .003$ & $\odot .0 \odot \odot$ & $\odot .0 \odot \odot$ & 2 \\
\hline $\begin{array}{l}\text { KRA@12 } \\
2\end{array}$ & 0.000 & 8.752 & $\odot .003$ & $\odot .0 \odot \odot$ & $\odot .00 \odot$ & 2 \\
\hline $\begin{array}{l}\text { KRA154 } \\
3\end{array}$ & $\odot .00 \odot$ & $\odot . \odot \odot \odot$ & 0.001 & $\odot .0 \odot \odot$ & $\odot .0 \odot \odot$ & 2 \\
\hline $\begin{array}{l}\text { KRA155 } \\
2\end{array}$ & $\odot .0 \odot \odot$ & 81.729 & $\odot .0 \odot 2$ & $\odot .0 \odot \odot$ & $\odot . \odot \odot \odot$ & 2 \\
\hline $\begin{array}{l}\text { KRA158 } \\
2\end{array}$ & $\odot .000$ & 79.402 & $\odot .0 \odot 2$ & $\odot .0 \odot \odot$ & $\odot .0 \odot \odot$ & 2 \\
\hline $\begin{array}{l}\text { KRA161 } \\
2\end{array}$ & $\odot .0 \odot \odot$ & 56.106 & $\odot .002$ & 0.000 & $\odot .00 \odot$ & 2 \\
\hline $\begin{array}{l}\text { KRA162 } \\
2\end{array}$ & $\odot . \odot \odot \odot$ & 96.748 & 0.002 & $\odot . \odot \odot \odot$ & $\odot . \odot \odot \odot$ & 2 \\
\hline $\begin{array}{l}\text { KRA164 } \\
2\end{array}$ & 0.000 & 64.956 & $\odot . \odot \odot 2$ & $\odot .000$ & $\odot .00 \odot$ & 2 \\
\hline $\begin{array}{l}\text { KRA169 } \\
2\end{array}$ & $\odot .000$ & 64.956 & $\odot .0 \odot 2$ & 0.000 & 0.000 & 2 \\
\hline $\begin{array}{l}\text { KRA172 } \\
\text { KR }\end{array}$ & $\odot . \odot \odot \odot$ & 93.775 & 0.002 & $\odot . \odot \odot \odot$ & $\odot . \odot \odot \odot$ & 2 \\
\hline
\end{tabular}

The following specimens are in the file ALCA4V2 Probabilities:

ID. NO. ALCA1V2 ALCA3V2 ALCA4V2 ALCA5V2 ALCA7V2 From:

Into:

$\begin{array}{lllllll}\text { KRA100 } & 0.00 \odot & 0.00 \odot & 25.322 & 0.00 \odot & 0.005 & 3\end{array}$

$\begin{array}{lllllll}3 & & & & & & \\ 3 & 0.000 & 0.00 \odot & 42.143 & 0.00 \odot & 0.001 & 3\end{array}$

$\begin{array}{lllllll}3 & & & & & \\ 3 & 0.000 & 0.000 & 55.020 & 0.000 & 0.001 & 3\end{array}$

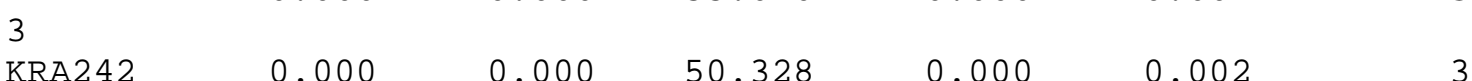

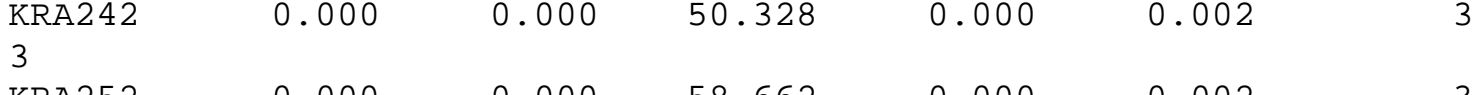

$\begin{array}{lllllll}\text { KRA252 } & \odot . \odot \odot \odot ~ & \odot . \odot \odot \odot ~ & 58.662 & \odot . \odot \odot \odot ~ & \odot . \odot \odot 2 & 3\end{array}$

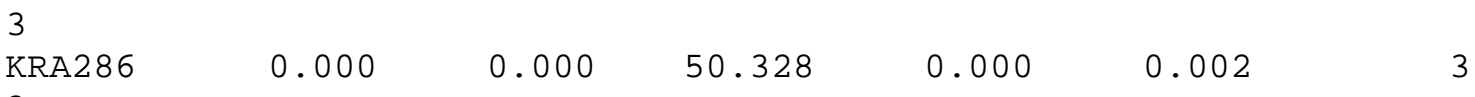

3

The following specimens are in the file ALCA5V2 


\begin{tabular}{|c|c|c|c|c|c|c|}
\hline & Probabi & ities: & & & & \\
\hline $\begin{array}{l}\text { ID. NO. } \\
\text { Into: }\end{array}$ & ALCA1V2 & ALCA3V2 & ALCA4V2 & ALCA5V2 & ALCA7V2 & From: \\
\hline $\begin{array}{l}\text { KRA190 } \\
4\end{array}$ & $\odot . \odot \odot \odot$ & $\odot . \odot \odot \odot$ & 0.026 & 98.641 & 0.028 & 4 \\
\hline $\begin{array}{l}\text { KRA106 } \\
4\end{array}$ & $\odot .000$ & $\odot . \odot \odot \odot$ & 0.031 & 68.877 & 0.050 & 4 \\
\hline $\begin{array}{l}\text { KRA107 } \\
4\end{array}$ & 0.000 & $\odot .0 \odot \odot$ & 0.018 & 70.196 & 0.012 & 4 \\
\hline $\begin{array}{l}\text { KRA109 } \\
4\end{array}$ & 0.000 & $\odot .0 \odot \odot$ & $\odot .022$ & 87.678 & 0.018 & 4 \\
\hline $\begin{array}{l}\text { KRA111 } \\
4\end{array}$ & 0.000 & $\odot .0 \odot \odot$ & $\odot .027$ & 57.870 & 0.045 & 4 \\
\hline $\begin{array}{l}\text { KRA112 } \\
4\end{array}$ & 0.000 & $\odot .00 \odot$ & $\odot .035$ & 7.913 & 0.112 & 4 \\
\hline $\begin{array}{l}\text { KRA209 } \\
4\end{array}$ & 0.000 & $\odot .00 \odot$ & $\odot .056$ & 8.559 & 0.028 & 4 \\
\hline $\begin{array}{l}\text { KRA211 } \\
4\end{array}$ & 0.000 & 0.000 & 0.018 & 72.799 & 0.017 & 4 \\
\hline $\begin{array}{l}\text { KRA212 } \\
4\end{array}$ & $\odot .000$ & $\odot .0 \odot \odot$ & $\odot .029$ & 91.129 & 0.032 & 4 \\
\hline $\begin{array}{l}\text { KRA217 } \\
4\end{array}$ & $\odot .0 \odot \odot$ & $\odot .0 \odot \odot$ & $\odot .017$ & 25.353 & $\odot .009$ & 4 \\
\hline $\begin{array}{l}\text { KRA219 } \\
4\end{array}$ & 0.000 & 0.000 & $\odot .022$ & 82.678 & 0.027 & 4 \\
\hline $\begin{array}{l}\text { KRA220 } \\
4\end{array}$ & 0.000 & 0.000 & 0.014 & 38.673 & 0.008 & 4 \\
\hline $\begin{array}{l}\text { KRA225 } \\
4\end{array}$ & 0.000 & $\odot . \odot \odot \odot$ & 0.015 & 20.701 & $\odot .0 \odot 7$ & 4 \\
\hline $\begin{array}{l}\text { KRA227 } \\
4\end{array}$ & 0.000 & $\odot . \odot \odot \odot$ & 0.021 & 94.127 & 0.019 & 4 \\
\hline $\begin{array}{l}\text { KRA229 } \\
4\end{array}$ & 0.000 & $\odot . \odot \odot \odot$ & $\odot .022$ & 59.569 & 0.015 & 4 \\
\hline $\begin{array}{l}\text { KRA231 } \\
4\end{array}$ & $\odot .0 \odot \odot$ & $\odot . \odot \odot \odot$ & 0.072 & 2.392 & 0.030 & 4 \\
\hline $\begin{array}{l}\text { KRA232 } \\
4\end{array}$ & 0.000 & $\odot .0 \odot \odot$ & ๑. 019 & 14.117 & 0.024 & 4 \\
\hline
\end{tabular}

The following specimens are in the file ALCA7V2 Probabilities:

\begin{tabular}{|c|c|c|c|c|c|c|}
\hline $\begin{array}{l}\text { ID. NO. } \\
\text { Into: }\end{array}$ & ALCA1V2 & LCA3V2 & ALCA4V2 & ALCA5V2 & ALCA7V2 & From: \\
\hline $\begin{array}{l}\text { KRA101 } \\
5\end{array}$ & $\odot . \odot \odot \odot$ & $\odot . \odot \odot \odot$ & $\odot .151$ & $\odot . \odot \odot \odot$ & 47.027 & 5 \\
\hline $\begin{array}{l}\text { KRA102 } \\
5\end{array}$ & $\odot . \odot \odot \odot$ & $\odot . \odot \odot \odot$ & $\odot .168$ & $\odot . \odot \odot \odot$ & 98.364 & 5 \\
\hline $\begin{array}{l}\text { KRA189 } \\
5\end{array}$ & $\odot .00 \odot$ & $0.00 \odot$ & $\odot .177$ & $\odot . \odot \odot \odot$ & 95.522 & 5 \\
\hline $\begin{array}{l}\text { KRA195 } \\
5\end{array}$ & $\odot .00 \odot$ & 0.000 & $\odot .281$ & $\odot . \odot \odot \odot$ & 38.294 & 5 \\
\hline $\begin{array}{l}\text { KRA197 } \\
5\end{array}$ & 0.000 & $0.00 \odot$ & 0.204 & $\odot . \odot \odot \odot$ & 42.040 & 5 \\
\hline KRA198 & 0.000 & $0.00 \odot$ & 0.133 & $\odot .001$ & 29.740 & 5 \\
\hline
\end{tabular}




$\begin{array}{lllllll}\text { KRA205 } & 0.000 & 0.00 \odot & 0.213 & 0.000 & 76.692 & 5 \\ 5 & 0.000 & 0.000 & 0.102 & 0.000 & 1.382 & 5 \\ \text { KRA208 } & 0.000 & 0.000 & 0.247 & 0.00 \odot & 33.551 & 5 \\ 5 & & & & & \end{array}$

Summary of Classification Success:

Into:

$\begin{array}{lrrrrrr}\text { From: } & \text { ALCA1V2 } & \text { ALCA3V2 } & \text { ALCA4V2 } & \text { ALCA5V2 } & \text { ALCA7V2 } & \text { Total } \\ \text { ALCA1V2 } & 19 & \odot & \odot & \odot & \odot & 19 \\ \text { ALCA3V2 } & \odot & 10 & 1 & \odot & \odot & 11 \\ \text { ALCA4V2 } & \odot & \odot & 6 & \odot & \odot & 6 \\ \text { ALCA5V2 } & \odot & \odot & \odot & 17 & \odot & 17 \\ \text { ALCA7V2 } & \odot & \odot & \odot & \odot & 9 & 9 \\ \text { Total } & 19 & 10 & 7 & 17 & 9 & 62\end{array}$


Table DR10. Mahalanobis distance results for P-XRF

MAHALANOBIS DISTANCE CALCULATION AND POSTERIOR CLASSIFICATION FOR TWO OR MORE GROUPS.

Date: $2 / 20 / 13$

File: PXRF Maha RbSR SD.txt

Groups are:

$\begin{array}{ll}1 & \text { SDALCA1 } \\ 2 & \text { SDALCA3 } \\ 3 & \text { SDALCA4 } \\ 4 & \text { SDALCA5 } \\ 5 & \text { SDALCA7 }\end{array}$

Variables used:

$\mathrm{Rb} \quad \mathrm{Sr}$

Probabilities are jackknifed for specimens included in each group.

The following specimens are in the file SDALCA1

Probabilities:

ID. NO SDALCA1 SDALCA3 SDALCA4 SDALCA5

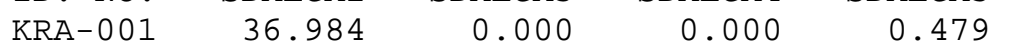

$\begin{array}{lllll}\text { KRA- } 026 & 81.766 & 0.000 & 0.000 & 0.521\end{array}$

$\begin{array}{lllll}\text { KRA- } 065 & 75.361 & 0.000 & 0.000 & 0.487\end{array}$

$\begin{array}{rrrrr}\text { KRA- }-7 \odot & 84.567 & 0.00 \odot & 0.00 \odot & 0.523 \\ \text { KRA- } 095 & 9.814 & 0.00 \odot & 0.00 \odot & 0.589\end{array}$

$\begin{array}{lllll}\text { KRA-113 } & 14.436 & 0.000 & 0.000 & 0.490\end{array}$

SDALCA7
$\odot .0 \odot \odot$

$\odot .000$

$\odot .000$

0.000

$\odot .000$

$\odot .0 \odot \odot$

The following specimens are in the file SDALCA3

ID. NO .

$\odot .000$

$\odot .000$

$\odot .000$

$\odot .000$

$\odot .000$

$\odot .000$

$\odot .000$

SDALCA3

44.642

47.006

62.898

95.034

53.930

KRA-162

KRA-164

\section{SDALCA5}

0.015

0.014

0.015

0.015

0.015

0.014

๑. 014
SDALCA7
$\odot .00 \odot$
$\odot .00 \odot$
$\odot .00 \odot$
$\odot .00 \odot$
$\odot .00 \odot$
$\odot .00 \odot$
$\odot .00 \odot$
SDALCA1
SDALCA4

0.001

0.001

0.001

0.001

0.001

0.001

$\odot .001$
From: Into:

$\begin{array}{rr}2 & 2 \\ 2 & 2\end{array}$

$2 \quad 2$

$2 \quad 2$

22

2

2

The following specimens are in the file SDALCA4 Probabilities:

ID. NO SDALCA1 SDALCA3

KRA- $\odot 97$

KRA- $\odot 98$

$\odot .00 \odot$

$\odot .00 \odot$

$\odot .00 \odot$

SDALCA4

29.969

SDALCA5

0.172

0.114

0.000

$\odot .000$

KRA -252

$\odot .000$

$\odot .000$

15.230

76.185

0.137

$\odot .000$

0.000

38.994

$\odot .000$

0.000

92.118

0.151

0.142
From: Into:

3

3

3

3

3

3

The following specimens are in the file SDALCA5 Probabilities:

ID. NO.

KRA- 104

KRA-108

SDALCA1

SDALCA3

$\odot .000$

$\odot .000$

SDALCA4

$\odot .002$

SDALCA5

SDALCA7
0.016
0.003
$\odot .019$

From

$\odot .002$

76.200

$\odot .00 \odot$

0.000

0.001

51.042

4

Into:

4

4 


\begin{tabular}{|c|c|c|c|c|c|c|}
\hline KRA-110 & 0.001 & 0.000 & 0.001 & 42.302 & 0.015 & 4 \\
\hline KRA-111 & $\odot .001$ & $\odot .00 \odot$ & 0.002 & 60.330 & $\odot .002$ & 4 \\
\hline KRA-112 & $\odot .001$ & $\odot .00 \odot$ & $0.0 \odot 2$ & 69.453 & $\odot .002$ & 4 \\
\hline \multicolumn{6}{|c|}{$\begin{array}{c}\text { The following specimens are in the file } \\
\text { Probabilities: }\end{array}$} & \\
\hline ID. NO. & SDALCA1 & SDALCA3 & SDALCA4 & SDALCA5 & SDALCA7 & From: \\
\hline KRA-101 & $\odot . \odot \odot \odot$ & $\odot . \odot \odot \odot$ & $\odot . \odot \odot \odot$ & 3.539 & 14.303 & 5 \\
\hline KRA-102 & $\odot . \odot \odot \odot$ & $\odot .0 \odot \odot$ & $\odot . \odot \odot \odot$ & 2.493 & 72.885 & \\
\hline KRA-189 & $\odot . \odot \odot \odot$ & $\odot . ๑ \odot \odot$ & $\odot . \odot \odot \odot$ & 1.727 & 22.521 & \\
\hline KRA-195 & ๑.००० & $\odot .000$ & ๑.००० & 2.314 & 87.746 & \\
\hline KRA-197 & $\odot .00 \odot$ & $\odot . \odot ० \odot$ & ๑.๑०९ & 1.758 & 71.433 & \\
\hline KRA-198 & $\odot .00 \odot$ & $\odot .00 \odot$ & $\odot .0 \odot \odot$ & 1.413 & 39.358 & \\
\hline KRA-205 & $\odot . \odot \odot \odot$ & $\odot . \odot \odot \odot$ & $\odot . \odot \odot \odot$ & 1.715 & 30.351 & \\
\hline \multicolumn{7}{|c|}{$\begin{array}{c}\text { Summary of Classification Success: } \\
\text { Into: }\end{array}$} \\
\hline From: & SDALCA1 & SDALCA3 & SDALCA4 & SDALCA5 & SDALCA7 & Total \\
\hline SDALCA1 & 6 & 0 & 0 & $\odot$ & 0 & 6 \\
\hline SDALCA3 & 0 & 7 & 0 & 0 & 0 & 7 \\
\hline SDALCA4 & 0 & 0 & 6 & $\Theta$ & $\odot$ & 6 \\
\hline SDALCA5 & 0 & 0 & 0 & 6 & 0 & 6 \\
\hline SDALCA7 & $\odot$ & 0 & $\odot$ & 0 & 7 & 7 \\
\hline Total & 6 & 7 & 6 & 6 & 7 & 32 \\
\hline
\end{tabular}

